\title{
Análise de diagnóstico em modelos semiparamétricos normais
}

\author{
Gleyce Rocha Noda \\ DisSERTAÇÃO APRESENTADA \\ $\mathrm{AO}$ \\ Instituto de Matemática e Estatística \\ DA \\ UNIVERSIDADE DE SÃO PAULO \\ PARA \\ OBTENÇÃO DO TÍTULO \\ $\mathrm{DE}$ \\ Mestre EM CiênCiAS \\ Programa: Estatística \\ Orientador: Prof. Dr. Gilberto Alvarenga Paula
}

Durante o desenvolvimento deste trabalho o autor recebeu auxílio financeiro da CAPES

São Paulo, maio de 2013 


\section{Análise de diagnóstico em modelos semiparamétricos normais}

Esta dissertação contém as correções e alterações sugeridas pela Comissão Julgadora durante a defesa realizada por Gleyce Rocha Noda em 18/04/2013.

O original encontra-se disponível no Instituto de Matemática e Estatística da Universidade de São Paulo.

Comissão Julgadora:

- Prof. Dr. Gilberto Alvarenga Paula (orientador) - IME-USP

- Prof. Dr. Francisco José de Azevêdo Cysneiros - UFPE

- $\operatorname{Prof}^{a} \operatorname{Dr}^{a}$ Cibele Maria Russo Noveli - ICMC-USP 


\section{Agradecimentos}

A realização desta dissertação de mestrado só foi possível graças à colaboração direta ou indireta de várias pessoas. Dentre essas, gostaria de agradecer em especial:

Ao meu orientador Gilberto Alvarenga Paula por toda dedicação e apoio dado durante o desenvolvimento deste trabalho.

Aos professores Francisco Cysneiros, Germán Ibacache e Raydonal Ospina pelos auxílios pontuais que precisei ao longo do desenvolvimento desta dissertação.

Aos meus pais, Seiki e Rosenilma, e aos meus irmãos Ylana e Renan, por me apoiarem durante esse período de dedicação acadêmica e por todo apoio, amor e paciência durante toda a minha formação.

Ao meu namorado Glauber De Bona pelo incentivo e entusiasmo contagiante pelo conhecimento, além da amizade, apoio e momentos felizes que passamos nos últimos anos.

Ao Tiago Pilan e Karin Tamura, que me ajudaram a conciliar a vida corporativa com a acadêmica, e a Karina Nakamura, amiga e companheira de mestrado.

Aos meus grandes amigos Tarsila Araújo, Frederico Miura e Ricardo Separovic pelos momentos de alegria, apoio e amizade.

Por fim, agradeço aos artistas plásticos que, com o objetivo de representar, imitar, expressar ou mesmo abstrair da realidade, tornam nossas vidas mais reflexivas e encantadoras. 


\section{Resumo}

\section{Análise de diagnóstico em modelos semiparamétricos normais}

Nesta dissertação apresentamos métodos de diagnóstico em modelos semiparamétricos sob erros normais, em especial os modelos semiparamétricos com uma variável explicativa não paramétrica, conhecidos como modelos lineares parciais. São utilizados splines cúbicos para o ajuste da variável resposta e são aplicadas funções de verossimilhança penalizadas para a obtenção dos estimadores de máxima verossimilhança com os respectivos erros padrão aproximados. São derivadas também as propriedades da matriz hat para esse tipo de modelo, com o objetivo de utilizá-la como ferramenta na análise de diagnóstico. Gráficos normais de probabilidade com envelope gerado também foram adaptados para avaliar a adequabilidade do modelo.

Finalmente, são apresentados dois exemplos ilustrativos em que os ajustes são comparados com modelos lineares normais usuais, tanto no contexto do modelo aditivo normal simples como no contexto do modelo linear parcial.

Palavras-chave: modelos não paramétricos, modelos lineares parciais, função de verossimilhança penalizada, splines cúbicos, suavizadores. 


\section{Abstract}

\section{Diagnostic analysis in semiparametric normal models}

In this master dissertation we present diagnostic methods in semiparametric models under normal errors, specially in semiparametric models with one nonparametric explanatory variable, also known as partial linear model. We use cubic splines for the nonparametric fitting, and penalized likelihood functions are applied for obtaining maximum likelihood estimators with their respective approximate standard errors. The properties of the hat matrix are also derived for this kind of model, aiming to use it as a tool for diagnostic analysis. Normal probability plots with simulated envelope graphs were also adapted to evaluate the model suitability.

Finally, two illustrative examples are presented, in which the fits are compared with usual normal linear models, such as simple normal additive and partially linear models.

Keywords: nonparametric models, partially linear models, penalized likelihood, cubic splines, smoothing. 


\section{Sumário}

Lista de Abreviaturas $\quad$ xi

Lista de Símbolos $\quad$ xiii

Lista de Figuras $\quad$ xv

Lista de Tabelas $\quad$ xvii

1 Introdução 1

1.1 Motivação . . . . . . . . . . . . . . . . . . . . . . . 2

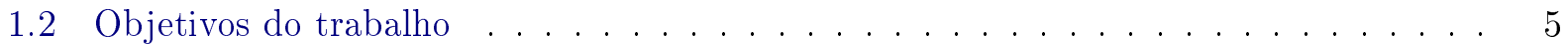

1.3 Organização do trabalho e suporte computacional . . . . . . . . . . . . 6

2 Modelos aditivos normais $\quad 9$

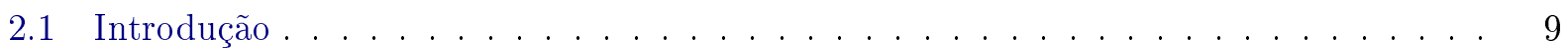

2.2 Especificação do modelo . . . . . . . . . . . . . . . . . . . . 9

2.3 Método de suavização spline cúbico . . . . . . . . . . . . . . . . . . . 10

2.4 Critério de penalização . . . . . . . . . . . . . . . . . . 11

2.4.1 Função de penalização . . . . . . . . . . . . . . . . . . . . . 12

2.4.2 Logaritmo da função de verossimilhança penalizada . . . . . . . . . . . . . . 13

2.4.3 Função escore e informação de Fisher penalizada . . . . . . . . . . . . . . . . 14

2.5 Estimação dos parâmetros . . . . . . . . . . . . . . . . . 15

2.5.1 Estimação da função não paramétrica . . . . . . . . . . . . . . . 15

2.5 .2 Graus de liberdade . . . . . . . . . . . . . . . . . . . 16

2.5.3 Estimação do parâmetro de dispersão . . . . . . . . . . . . . . . . 17

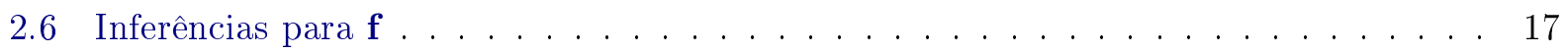

2.6 .1 Distribuição de $\widehat{\mathbf{f}} \ldots \ldots \ldots \ldots \ldots \ldots \ldots \ldots \ldots$

2.6 .2 Erro padrão . . . . . . . . . . . . . . . . . . . . . 18

2.6 .3 Testes de hipóteses . . . . . . . . . . . . . . . . . . . 19

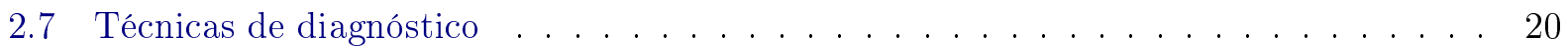

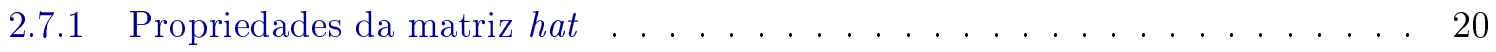

2.7 .2 Pontos de alavanca . . . . . . . . . . . . . . . . . . . . 23

2.7 .3 Resíduos . . . . . . . . . . . . . . . . . . . 23 
2.7 .4 Influência . . . . . . . . . . . . . . . . . . . . . . 24

2.8 Estimação do parâmetro de suavização . . . . . . . . . . . . . . . . . 24

2.8 .1 Validação cruzada . . . . . . . . . . . . . . . . . . 25

2.8 .2 Validação cruzada generalizada . . . . . . . . . . . . . . . . . . . . . . . . . . . . .

2.9 Seleção de modelos . . . . . . . . . . . . . . . . . . . . . . 26

2.10 Suavizadores ponderados . . . . . . . . . . . . . . . . 26

2.11 Observações empatadas . . . . . . . . . . . . . . . . . . . . . . . . . . . . . . . . .

2.12 Considerações . . . . . . . . . . . . . . . . . . . . . 28

3 Modelos semiparamétricos normais $\quad 29$

3.1 Introdução . . . . . . . . . . . . . . . . . . . . . . . . . 29

3.2 Especificação do modelo . . . . . . . . . . . . . . . . . . . 30

3.3 Critério de penalização . . . . . . . . . . . . . . . . . 31

3.3 .1 Função de penalização . . . . . . . . . . . . . . . . . . . 31

3.3.2 Logaritmo da função de verossimilhança penalizada . . . . . . . . . . . . . . . 32

3.3.3 Função escore e informação de Fisher penalizada . . . . . . . . . . . . . . . . 32

3.4 Estimação dos parâmetros . . . . . . . . . . . . . . . . . . . . 33

3.4 .1 Estimação dos parâmetros de posição . . . . . . . . . . . . . . . . 33

3.4 .2 Graus de liberdade . . . . . . . . . . . . . . . . . . . . 35

3.4.3 Estimação do parâmetro de dispersão . . . . . . . . . . . . . . . . 35

3.4 .4 Estimação do parâmetro de suavização . . . . . . . . . . . . . . . 36

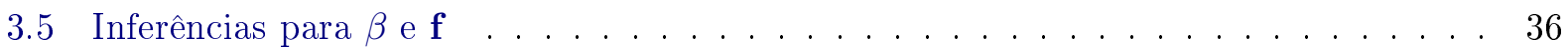

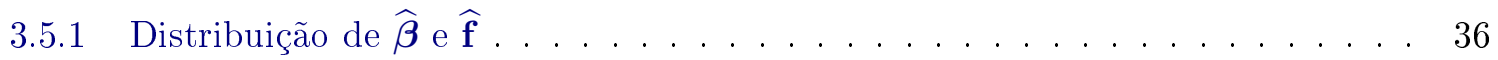

3.5.2 Erro padrão . . . . . . . . . . . . . . . . . . 37

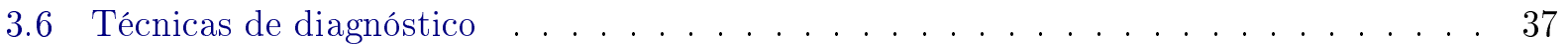

3.6 .1 Decomposição da matriz $\overline{\mathbf{H}}(\lambda) \ldots \ldots \ldots \ldots \ldots$

3.6 .2 Alavanca . . . . . . . . . . . . . . . . . . . . 39

3.6 .3 Resíduos . . . . . . . . . . . . . . . . . . 39

3.6 .4 Influência . . . . . . . . . . . . . . . . . . . . . 39

3.7 Seleção de modelos . . . . . . . . . . . . . . . . . . . . . . . . . . 40

3.8 Considerações . . . . . . . . . . . . . . . . . . . . . . . . . 41

4 Aplicações $\quad 43$

4.1 MANS: Saída elétrica . . . . . . . . . . . . . . . . . . . 43

4.1 O modelo . . . . . . . . . . . . . . . . . . . . 44

4.1 .2 Ajuste do MANS . . . . . . . . . . . . . . . . . 44

4.1 .3 Diagnóstico e ajustes adicionais . . . . . . . . . . . . . . . . 45

4.2 MLP: Snacks . . . . . . . . . . . . . . . . . . . . . . . 49

$4.2 .1 \quad \mathrm{O}$ modelo . . . . . . . . . . . . . . . . . . . 50

4.2 .2 Ajuste do MLP . . . . . . . . . . . . . . . . . 50

4.2 .3 Diagnóstico e ajustes adicionais . . . . . . . . . . . . . . . . 52 
5 Conclusões $\quad \mathbf{5 7}$

5.1 Considerações finais . . . . . . . . . . . . . . . . . . . . 57

5.2 Implementação computacional . . . . . . . . . . . . . . . . . . . 58

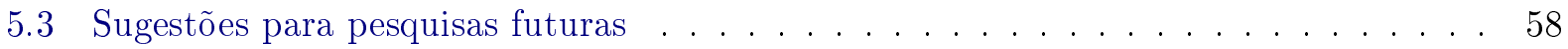

A Dados Saída elétrica $\quad 59$

B Derivações do logaritmo da função de verossimilhança penalizada no MANS 61

B.1 Derivadas de primeira ordem . . . . . . . . . . . . . . . . . 61

B.2 Derivadas de segunda ordem . . . . . . . . . . . . . . . 62

C Derivações do logaritmo da função de verossimilhança penalizada no MLP 65

C.1 Derivadas de primeira ordem . . . . . . . . . . . . . . 65

C.2 Derivadas de segunda ordem . . . . . . . . . . . . . 66

$\begin{array}{ll}\text { D Códigos em R } & 69\end{array}$

D.1 Obtenção da matriz hat no MANS . . . . . . . . . . . . . . . . . . 69

D.2 Gráfico Envelope para o MANS ajustado via gamlss . . . . . . . . . . . 71

D.3 Gráficos de diagnóstico para o MANS ajustado via gamlss . . . . . . . . . . 72

D.4 Gráfico Envelope para o MLP ajustado via gam . . . . . . . . . . . . . . . 73

D.5 Gráficos de diagnóstico para o MLP ajustado via gam . . . . . . . . . . . . . 74

$\begin{array}{ll}\text { Referências Bibliográficas } & 77\end{array}$ 


\section{Lista de Abreviaturas}

\begin{tabular}{ll}
\hline EMVP & Estimador de Máxima Verossimilhança Penalizado \\
GAM & Generalized Additive Models \\
GAMLSS & Generalized Additive Models for Location, Scale and Shape \\
GLE & Grau de Liberdade Efetivo \\
MAG & Modelo Aditivo Generalizado \\
MAN & Modelo Aditivo Normal \\
MANS & Modelo Aditivo Normal Simples \\
MLG & Modelo Linear Generalizado \\
MLP & Modelo Linear Parcial \\
MP & Modelo Paramétrico \\
SCN & Spline Cúbico Natural \\
SQEP & Soma dos Quadrados dos Erros Penalizados \\
VC & Validação Cruzada \\
VCG & Validação Cruzada Generalizada \\
\hline
\end{tabular}


xii LISTA DE ABREVIATURAS 


\section{Lista de Símbolos}

\begin{tabular}{ll}
\hline $\mathbf{A}, \mathbf{N}, \mathbf{X}, \mathbf{H}, \ldots$ & Matrizes reais \\
$\mathbf{y}, \mathbf{x}, \ldots$ & Vetores constantes \\
$\alpha, \beta, \gamma, \delta$ & Parâmetros \\
$\boldsymbol{\theta}, \mathbf{f}, \boldsymbol{\beta}$ & Vetores de parâmetros \\
$\mathbf{A}^{T}$ & Transposta da matriz $\mathbf{A}$ \\
$\mathbf{A}^{-1}$ & Inversa da matriz $\mathbf{A}$ \\
$\mathbf{A}^{-}$ & Inversa generalizada da matriz $\mathbf{A}$ \\
$\operatorname{tr}\{\mathbf{A}\}$ & Traço da matriz $\mathbf{A}$ \\
\hline
\end{tabular}


xiv

LISTA DE SÍMBOLOS 


\section{Lista de Figuras}

1.1 Perfis médios do logaritmo da força de cisalhamento para os 5 tipos de snacks ao longo das semanas. . . . . . . . . . . . . . . . . . . . 3

1.2 Gráfico de densidade do logaritmo da variável força de cisalhamento. . . . . . . . . 3

1.3 Gráficos boxplots do logaritmo da força de cisalhamento ao longo das semanas por grupo para os dados sobre snacks. . . . . . . . . . . . . . . . . . . 4

1.4 Gráfico normal de probabilidades com envelope gerado para o resíduo studentizado referente ao grupo do modelo normal linear aos dados sobre snacks. . . . . . . . . . . 5

1.5 Gráficos de diagnóstico referentes ao modelo normal linear ajustado aos dados sobre

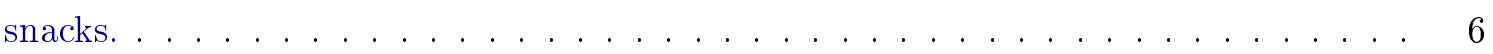

4.1 Diagrama de dispersão entre a saída elétrica e a velocidade do vento. . . . . . . . . . 43

4.2 Gráficos dos modelos ajustados para diferentes graus de liberdade aos dados de saída elétrica. . . . . . . . . . . . . . . . . . . . . . 45

4.3 Gráficos dos valores obtidos para o critério de informação de Schwarz à medida que aumentamos os graus de liberdade do ajuste para os dados de saída elétrica. . . . . . 46

4.4 Gráfico normal de probabilidades com envelope gerado para o resíduo ordinário padronizado referente ao ajuste do MANS para os dados de saída elétrica. . . . . . . . 47

4.5 Gráficos de diagnóstico referentes ao MANS ajustado aos dados de saída elétrica. . . 47

4.6 Gráfico normal de probabilidades com envelope gerado para o resíduo ordinário padronizado referente ao ajuste do MANS para os dados de saída elétrica sem a observação

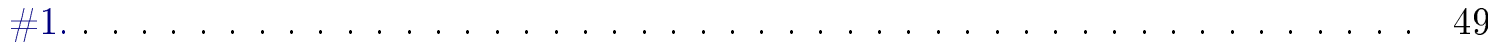

4.7 Gráficos de diagnóstico referentes ao MANS ajustado aos dados de saída elétrica sem a observação \#1. . . . . . . . . . . . . . . . . . . . . . . . . . . . . . . 49

4.8 MANS ajustado aos dados de saída elétrica. . . . . . . . . . . . . . . 50

4.9 Perfis ajustados dos modelos MP e MLP referentes aos dados sobre snacks. . . . . . 52

4.10 Gráfico normal de probabilidades com envelope gerado para o resíduo ordinário padronizado referente ao ajuste do MLP aos dados sobre snacks. . . . . . . . . . . . . 53

4.11 Gráficos de diagnóstico referentes ao modelo MLP ajustado aos dados sobre snacks. . 53

4.12 Gráfico normal de probabilidades com envelope gerado para o resíduo ordinário padronizado referente ao ajuste do MLP sem a observação \#452 ajustado aos dados sobre snacks . . . . . . . . . . . . . . . . . . . . . 54 
4.13 Gráficos de diagnóstico referentes ao modelo MLP sem a observação \#452 ajustado aos dados sobre snacks . . . . . . . . . . . . . . . . . . . 55 


\section{Lista de Tabelas}

1.1 Descrição das composições dos grupos de snacks. . . . . . . . . . . . . . . . 2

1.2 Estimativas dos parâmetros com os erros padrão aproximados referentes ao modelo normal linear ajustados aos dados sobre snacks. . . . . . . . . . . . . . 4

1.3 Desvio global e critérios de informação referentes ao modelo normal linear ajustado aos dados sobre snacks. . . . . . . . . . . . . . . . 5

$3.1(u+1)$-ésima etapa do algoritmo backfitting sob o MLP. . . . . . . . . . . 34

4.1 Valores estimados do logaritmo da função de verossimilhança penalizada e do critério de informação de Schwarz para diferentes graus de liberdade para os dados de saída elétrica. . . . . . . . . . . . . . . . . . . . . 44

4.2 Estimativas e erros padrão da função não paramétrica e do parâmetro de dispersão para o modelo ajustado aos dados de saída elétrica. . . . . . . . . . . . . . 46

4.3 Estimativas e erros padrão da função não paramétrica e do parâmetro de dispersão para o modelo ajustado aos dados de saída elétrica sem a observação $\# 1$. . . . . . . 48

4.4 Comparação do desvio global e critérios de informação para o modelo completo e o modelo sem a observação \#1 para os dados de saída elétrica. . . . . . . . . . . . . 48

4.5 Estimativas de máxima verossimilhança penalizada dos efeitos dos tipos de snacks com os respectivos erros padrão aproximados para os modelos MP e MLP ajustados as dados sobre snacks. . . . . . . . . . . . . . . . . . 51

4.6 Estimativas de máxima verossimilhança penalizadas com os respectivos erros padrão aproximados dos componentes não paramétricos referentes ao modelo MLP ajustados aos dados sobre snacks. . . . . . . . . . . . . . . . . . . . 51

4.7 Comparação entre as estimativas do MLP completo e do MLP sem a observação \#452 no ajuste aos dados sobre snacks. . . . . . . . . . . . . . . . 54 54

4.8 Comparação entre os GLEs, parâmetro de suavização e parâmetro de dispersão dos MLPs completo e sem a observação \#452 no ajuste aos dados sobre snacks. . . . . . 54

A.1 Dados de saída elétrica. . . . . . . . . . . . . . . . . . . 59 
xviii LISTA DE TABELAS 


\section{Capítulo 1}

\section{Introdução}

Os modelos estatísticos formam um conjunto muito útil de ferramentas que permitem estudar relações entre variáveis aleatórias. As formas mais simples dessas relações são as lineares, e durante muitos anos os modelos normais lineares foram utilizados na tentativa de descrever a maioria dessas relações.

No entanto, diversos estudos já realizados mostram que a suposição de linearidade entre a variável resposta e as variáveis explicativas nem sempre é apropriada. Nesses casos, alguns autores propõem quantificar o efeito dessas variáveis através de funções arbitrárias, de forma que a suposição de linearidade seja relaxada e o modelo permita maior flexibilidade na relação entre as variáveis. Porém, essa flexibilidade requer alguns cuidados, já que precisamos escolher que tipo de aproximação de funções é mais adequado, os graus dos polinômios que melhor representam essas relações e quanto essas funções arbitrárias podem influenciar na qualidade do ajuste do modelo.

Dentre as funções arbitrárias, os splines formam as ferramentas mais utilizadas atualmente. Os splines são oriundos do cálculo numérico que ganhou atenção na área da Estatística justamente pelo seu poder adaptativo na aproximação de funções. Neste trabalho, serão estudados especialmente os splines cúbicos.

O acréscimo de uma função não paramétrica no modelo exige o trabalho de comprovar se as suposições e as técnicas utilizadas para a inferência dos parâmetros, ajuste de modelos e análise de diagnóstico são equivalentes aos utilizados nos modelos lineares usuais, em que toda a teoria já está bem consolidada. Nesse caso, veremos que a estimação via mínimos quadrados e máxima verossimilhança terão de ser adaptadas, assim como a definição de graus de liberdade e de matriz projetora equivalentes aos que existem atualmente nos modelos lineares usuais.

O objetivo deste trabalho é apresentar toda a teoria referente aos modelos aditivos normais simples, ou seja, modelos normais com uma variável explicativa de comportamento não paramétrico, e posteriormente estudar modelos mais utilizados na prática, que são os modelos que consideram uma variável explicativa não paramétrica e variáveis explicativas de comportamento paramétrico para explicar algum fenômeno de interesse. Estudos mostram que esse tipo de modelo é muito utilizado em pesquisas que envolvem dados longitudinais. Outro objetivo é estudar técnicas de diagnóstico para esses modelos e fazer a implementação computacional no R para modelos ajustados via pacotes 
gam e gamlss.

\subsection{Motivação}

Vamos considerar como exemplo motivacional parte dos dados de um experimento desenvolvido no Departamento de Nutrição da Faculdade de Saúde Pública da USP, em que 5 formas diferentes de um novo tipo de snack, com baixo teor de gordura saturada e de ácidos graxos, foram comparados ao longo de 20 semanas (vide Paula et al. [2004]). Neste novo produto optou-se por substituir, totalmente ou parcialmente, a gordura vegetal hidrogenada (agente responsável pela fixação do aroma do produto), por óleo de canola. As 5 novas composições dos snacks são apresentadas na Tabela 1.1.

Tabela 1.1: Descrição das composições dos grupos de snacks.

\begin{tabular}{lcc}
\hline Grupo & \% Gordura & \% Óleo de Canola \\
\hline 1 (A) & $22 \%$ & $0 \%$ \\
2 (B) & $0 \%$ & $22 \%$ \\
3 (C) & $17 \%$ & $5 \%$ \\
4 (D) & $11 \%$ & $11 \%$ \\
$5(\mathrm{E})$ & $5 \%$ & $17 \%$ \\
\hline
\end{tabular}

O experimento foi conduzido de modo que nas semanas pares 15 embalagens de cada grupo de produtos A, B, C, D e E fossem analisadas em laboratório, totalizando 750 observações. Dentre as diversas variáveis observadas ao longo das semanas, estudaremos o comportamento da textura dos produtos através da força necessária para o cisalhamento, ou seja, a força necessária para cortar o snack (medida em Newton), com o objetivo de verificar se o efeito da composição e da semana alteram o aspecto de textura do produto.

Fazendo a análise preliminar da força necessária para o cisalhamento, percebe-se que sua distribuição segue uma distribuição gama, e dessa forma consideraremos o logaritmo da força necessária para o cisalhamento, de forma a satisfazer a suposição de normalidade da variável resposta.

A Figura 1.1 mostra que a força de cisalhamento parece maior nos grupos A e C, que são os grupos que possuem maiores percentuais de gordura na composição. Além disso, a figura também mostra um comportamento crescente da força de cisalhamento até a $14^{a}$ semana seguida de queda nas últimas três, sugerindo um comportamento não linear entre essas variáveis. Indícios de simetria podem ser observados no gráfico de densidade do logaritmo da força de cisalhamento, vide Figura 1.2 .

Já na Figura 1.3, percebe-se que o comportamento do logaritmo da força de cisalhamento ao longo das semanas altera bastante quando observamos cada grupo isoladamente, e os grupos de composição D e E são os que possuem menor variabilidade no logaritmo da força de cisalhamento ao longo das semanas. Os demais grupos parecem ter uma variabilidade maior.

Dado o comportamento não linear e a simetria dos dados, vamos supor um modelo inicial em que 


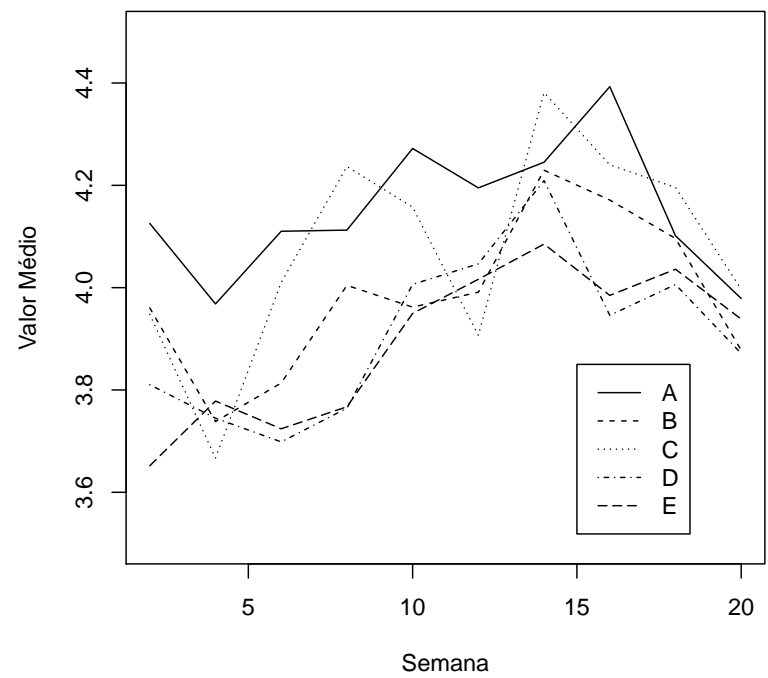

Figura 1.1: Perfis médios do logaritmo da força de cisalhamento para os 5 tipos de snacks ao longo das semanas.

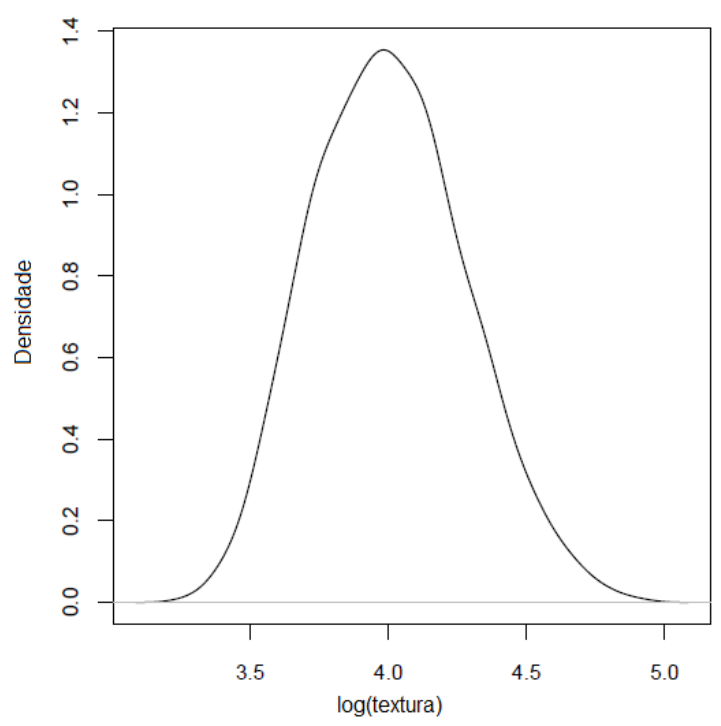

Figura 1.2: Gráfico de densidade do logaritmo da variável força de cisalhamento.

$y_{i j k}$ denota o valor do logaritmo da força de cisalhamento referente à $k$-ésima réplica $(k=1, \ldots, 15)$ do $i$-ésimo grupo $(i=1, \ldots, 5)$ na $j$-ésima semana $(j=2,4,6, \ldots, 20)$, e que $y_{i j k}$ são independentes com distribuição normal de média $\mu_{i j}$ e variância $\sigma^{2}$. Esse modelo pode ser escrito como

$$
y_{i j k}=\alpha+\beta_{i}+\gamma \times \operatorname{semana}_{j}+\delta \times \operatorname{semana}_{j}^{2}+\epsilon_{i j k},
$$

em que $\beta_{1}=0$, assim $\alpha, \alpha+\beta_{2}, \alpha+\beta_{3}$ e $\alpha+\beta_{4}$ e $\alpha+\beta_{5}$ denotam os efeitos dos snacks $\mathrm{A}, \mathrm{B}, \mathrm{C}$, D e E, respectivamente, a variável semana está sendo controlada de forma quadrática e $\epsilon_{i j k}$ é um 

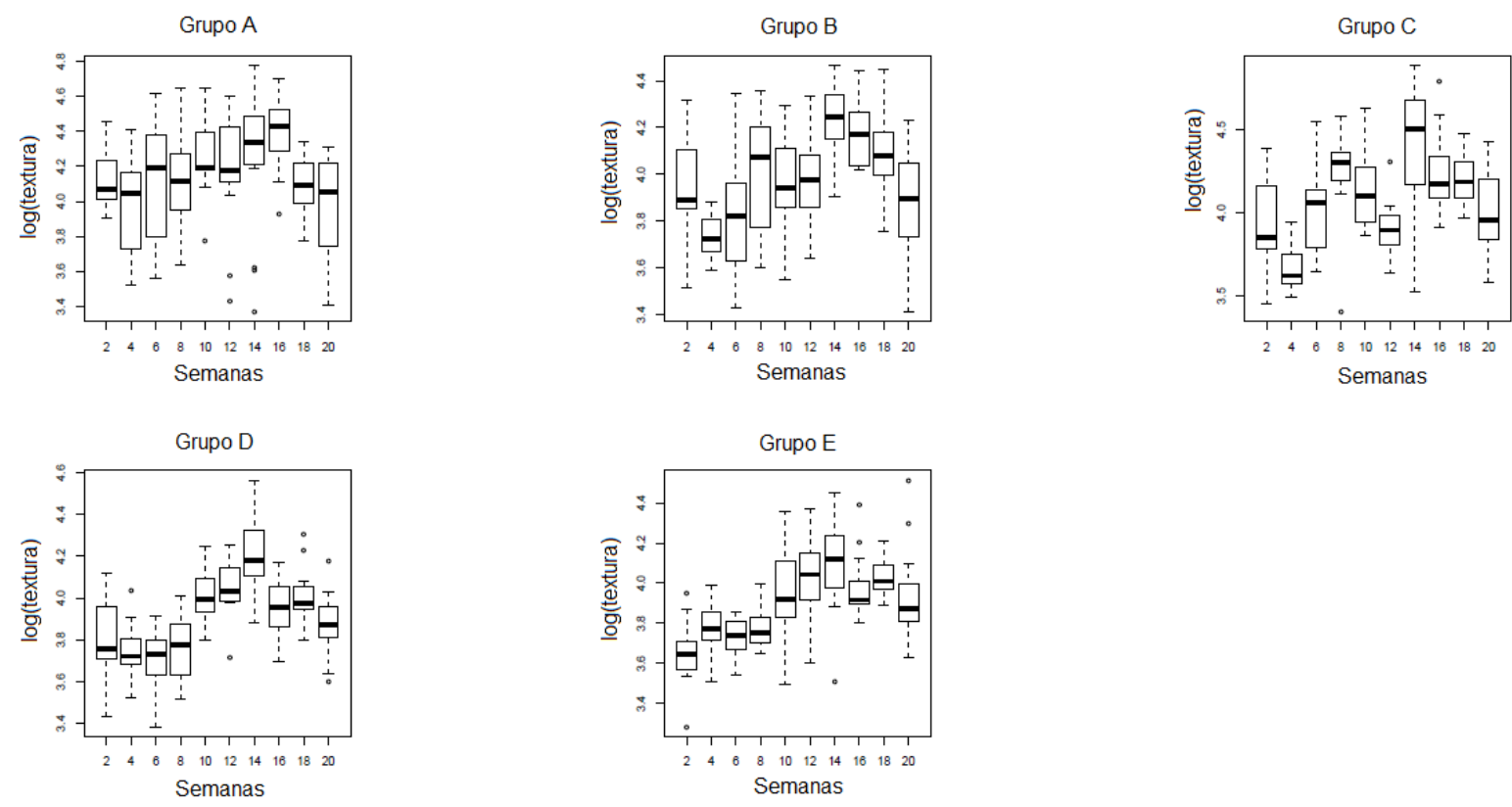

Figura 1.3: Gráficos boxplots do logaritmo da força de cisalhamento ao longo das semanas por grupo para os dados sobre snacks.

erro aleatório não observável de distribuição $\mathcal{N}\left(0, \sigma^{2}\right)$.

As estimativas dos parâmetros do modelo proposto e as estimativas dos critérios de seleção de modelos são apresentadas na Tabela 1.2. Note que todos os efeitos são significativos ao nível de 5\%. Já na Tabela 1.2 temos as medidas de desvio global do ajuste $(-2 \ell(\boldsymbol{\theta})$, com $\ell(\boldsymbol{\theta})$ sendo o logaritmo da função de verossimilhança), as derivações do critério de informação de Akaike [1974] e de Schwarz [1978].

Tabela 1.2: Estimativas dos parâmetros com os erros padrão aproximados referentes ao modelo normal linear ajustados aos dados sobre snacks.

\begin{tabular}{lrrr}
\hline & Estimativa & Erro Padrão & Valor $t$ \\
\hline Intercepto & 3,805 & 0,036 & 104,533 \\
Grupo B & $-0,166$ & 0,027 & $-6,081$ \\
Grupo C & $-0,076$ & 0,027 & $-2,798$ \\
Grupo D & $-0,240$ & 0,027 & $-8,799$ \\
Grupo E & $-0,257$ & 0,027 & $-9,430$ \\
Semana & 0,062 & 0,006 & 9,278 \\
Semana $^{2}$ & $-0,002$ & $0,296 \times 10^{-3}$ & $-7,405$ \\
$\sigma^{2}$ & 0,052 & 0,026 & $-55,91$ \\
\hline
\end{tabular}

O gráfico normal de probabilidades com envelope simulado para o resíduo studentizado (Figura 1.4) não fornece indícios de que o modelo não está bem ajustado, porém os gráficos de diagnóstico (Figura 1.5) indicam que existe uma série de pontos possivelmente influentes, além de indícios de não homogeneidade na variância dos erros. Dentre esses pontos, podemos destacar as observações 
Tabela 1.3: Desvio global e critérios de informação referentes ao modelo normal linear ajustado aos dados sobre snacks.

\begin{tabular}{lr}
\hline Critério de seleção & Valor \\
\hline$-2 \ell(\boldsymbol{\theta})$ & $-37,063$ \\
AIC & $-21,063$ \\
SIC & 15,898 \\
\hline
\end{tabular}

\#682 e \#688, que têm composição pertencente ao grupo A e possuem força de cisalhamento muito menor na $20^{a}$ semana quando comparada com a composição das demais observações com as mesmas características. Os pontos \#452 e \#384 também pertencem à composição A e suas forças de cisalhamento foram medidas, respectivamente, na $12^{a}$ e $14^{a}$ semanas, e observou-se uma força de cisalhamento menor que a esperada.

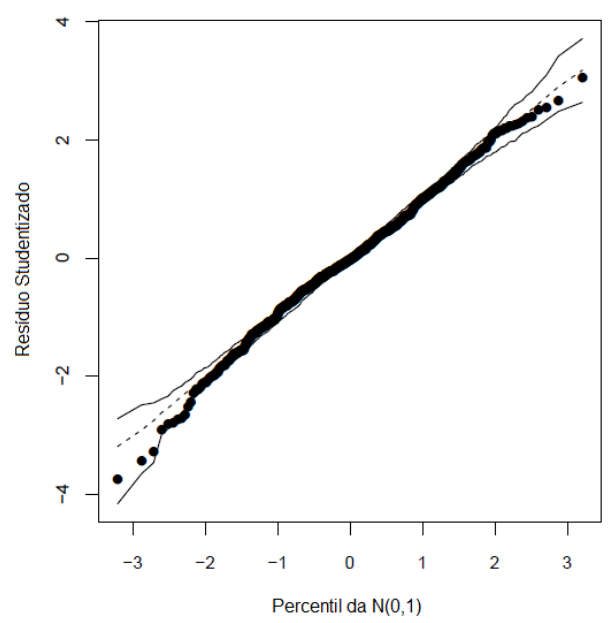

Figura 1.4: Gráfico normal de probabilidades com envelope gerado para o resíduo studentizado referente ao grupo do modelo normal linear aos dados sobre snacks.

Uma alternativa ao modelo proposto em (1.1) é tentarmos ajustar a variável semana de forma não paramétrica através de splines cúbicos, já que esse tipo de modelo não exige uma relação linear entre a variável resposta e a variável explicativa. Dessa forma, estudaremos os modelos que consideram a inclusão uma variável não paramétrica e voltaremos a esse exemplo motivacional no final deste trabalho assumindo um modelo em que a variável semana é considerada como uma covariável não paramétrica, e os resultados relacionados à estimação dos parâmetros, adequabilidade do ajuste e desempenho serão comparados.

\subsection{Objetivos do trabalho}

Nesta dissertação temos como objetivo apresentar os modelos aditivos normais simples e os modelos semiparamétricos sob erros normais, com o objetivo de resgatar toda a teoria referente a 

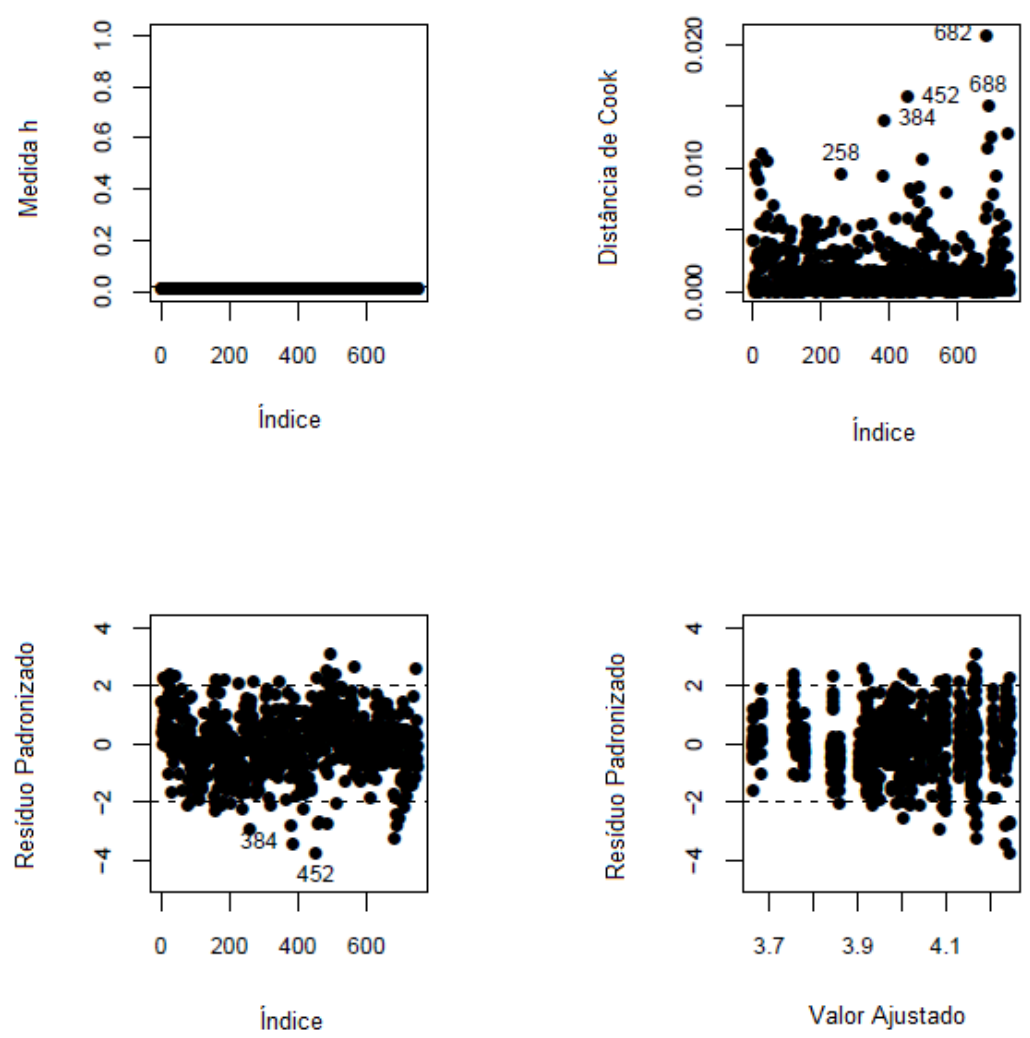

Figura 1.5: Gráficos de diagnóstico referentes ao modelo normal linear ajustado aos dados sobre snacks.

esses modelos na literatura. Também serão implementadas as matrizes projetoras dos MANSs para dados ajustados via gamlss com o objetivo de utilizá-las como ferramenta na análise de diagnóstico. Gráficos de diagnóstico serão adaptados para avaliação da adequabilidade dos modelos propostos.

\subsection{Organização do trabalho e suporte computacional}

Este texto está estruturado da seguinte forma: no Capítulo 2 são introduzidos os modelos aditivos normais simples, com o objetivo de apresentar alguns conceitos básicos para esse tipo de modelo, como a especificação das variáveis, ajuste via splines cúbicos e introdução ao parâmetro de suavização, além das definições de funções de verossimilhança penalizada e da teoria referente à estimação, graus de liberdade, análise de resíduos e diagnósticos para esse tipo de modelo.

No Capítulo 3 estendemos toda a teoria vista no Capítulo 2 para modelos com variáveis explicativas paramétricas e uma variável não paramétrica, denominados modelos semiparamétricos ou modelos lineares parciais com erros normais. Compõem esse capítulo a estimação dos parâmetros através da verossimilhança penalizada pelo método iterativo backfitting e pelo método direto, estudo dos graus de liberdade, estimação dos parâmetros de dispersão e suavização, análise de resíduos e técnicas de diagnóstico que permitem a análise de pontos de alavanca e influentes no componente paramétrico, no componente não paramétrico e na média. 
Já o Capítulo 4 ilustra a teoria apresentada em 2 aplicações: a primeira através de dados obtidos de Montgomery et al. [2001], em que um engenheiro deseja investigar a relação entre a velocidade do vento (mph) e o valor de saída elétrica de um moinho, que utilizaremos para ilustrar as inferências e diagnóstico nos modelos aditivos normais simples; e a segunda é uma aplicação em dados reais obtidas de Paula et al. [2004], revisitando o exemplo motivacional deste capítulo através do ajuste via modelo linear parcial sob erros normais em comparação ao modelo ajustado de forma paramétrica. Por fim, é apresentado no Capítulo 5 uma discussão sobre resultados, conclusões e possíveis pesquisas futuras deste trabalho.

Nos Apêndices A, B e C temos, respectivamente, a base de dados utilizada para a modelagem da saída elétrica do Capítulo 4, as derivadas de primeira e segunda ordens para a obtenção do vetor escore e a matriz de informação de Fisher para os modelos apresentados no Capítulo 2 e as derivadas de primeira e segunda ordens para a obtenção do vetor escore e a matriz de informação de Fisher para os modelos apresentados no Capítulo 3.

Finalmente, os códigos desenvolvidos ao longo desta dissertação para a estimação e diagnóstico dos modelos, assim como os gráficos foram produzidos utilizando o $\mathrm{R}$ (disponível gratuitamente em http://r-project.org/) e encontram-se no Apêndice D. Para maiores detalhes sobre o R, ver Ihaka e Gentleman [1996] e Cribari-Neto e Zarkos [1999]. 


\section{Capítulo 2}

\section{Modelos aditivos normais}

\subsection{Introdução}

Os modelos aditivos normais são uma extensão do modelo normal linear usual, em que são adotadas funções não paramétricas para estudar a relação entre a variável resposta e as variáveis explicativas. Esse tipo de modelo permite maior flexibilidade na modelagem da relação entre essas variáveis, pois não requer uma forma rígida de dependência entre elas.

Dentre os principais trabalhos relacionados aos modelos aditivos normais, temos Buja et al. [1989], Hastie e Tibshirani [1990] e Green e Silverman [1994], que estudaram detalhadamente esses modelos no contexto unidimensional, multidimensional e semiparamétrico. Hastie e Tibshirani [1990] também fizeram a generalização desse tipo de modelo para a família exponencial, criando os Modelos Aditivos Generalizados (MAG). Wood [2006] também estudou essa classe de modelos, porém concentrando-se em implementações no software R. Eubank [1988]), Craven e Wahba [1978] e Kim [1996] realizaram diversos estudos no que diz respeito às técnicas de diagnóstico possíveis para esse tipo de modelo. Já Rigby e Stasinopoulos [2005] desenvolveram os GAMLSS (Generalized Additive Models for Location, Scale and Shape), em que algumas suposições dos Modelos Lineares Generalizados (MLGs) e MAGs são relaxadas, além da possibilidade de estimação não só de parâmetros de posição e dispersão, mas também de assimetria e curtose. Além disso, os autores também desenvolveram o pacote gamlss no R, o qual será utilizado em uma das aplicações deste trabalho.

Como um dos objetivos deste trabalho é estudar métodos de diagnóstico em modelos semiparamétricos normais com uma função não paramétrica, este capítulo tem como objetivo introduzir toda a teoria referente ao modelo aditivo normal simples (MANS), e no próximo capítulo será feita a extensão para os modelos semiparamétricos.

\subsection{Especificação do modelo}

Seja $y_{i}(i=1, \ldots, n)$ o valor da variável resposta associada à $i$-ésima observação de uma amostra. O MANS assume a forma

$$
y_{i}=f\left(t_{i}\right)+\epsilon_{i}
$$


em que $t_{i}$ é o valor da variável explicativa da $i$-ésima observação, $f(\cdot)$ é uma função univariada arbitrária não especificada do efeito não paramétrico, e $\epsilon_{i}$ é o erro aleatório não observável com distribuição normal de média zero e variância $\sigma^{2}$ desconhecida. Os erros são assumidos mutuamente independentes. Nesse caso, qualquer estimativa de $f\left(t_{i}\right)$ será uma estimativa da esperança condicional $\mathrm{E}\left(Y_{i} \mid T=t_{i}\right)$.

Considerando a amostra de $n$ observações, podemos escrever o modelo matricialmente como

$$
\mathbf{y}=\mathbf{N f}+\epsilon
$$

sendo $\mathbf{y}=\left(y_{1}, \ldots, y_{n}\right)^{T}$ o vetor $(n \times 1)$ de respostas observadas independentes, $\mathbf{f}$ é o vetor de funções $(r \times 1)$ do efeito não paramétrico definido como

$$
\mathbf{f}=\left(\begin{array}{c}
f\left(t_{1}^{0}\right) \\
\vdots \\
f\left(t_{r}^{0}\right)
\end{array}\right)
$$

em que $t_{j}^{0}$ são os valores ordenados e distintos da variável explicativa, com $j=1, \ldots, r$ e $r=n$ quando não há empates nos valores dessa variável. $\mathbf{N}$ é a matriz de incidência $(n \times r)$, com elementos dados pela função indicadora $I\left(t_{i}=t_{j}^{0}\right), \operatorname{com} \mathbf{N}=\mathbf{I}_{n}$ quando não há empates. Finalmente, $\boldsymbol{\epsilon}=\left(\epsilon_{1}, . ., \epsilon_{n}\right)^{T}$ é o vetor de erros aleatórios $(n \times 1)$ normais de média zero e variância $\sigma^{2}$. Logo, temos as distribuições de $\mathbf{y}$ e $\boldsymbol{\epsilon}$ dadas por

$$
\left\{\begin{array}{l}
\mathbf{y} \sim \mathcal{N}_{n}\left(\mathbf{N f}, \sigma^{2} \mathbf{I}\right) \\
\boldsymbol{\epsilon} \sim \mathcal{N}_{n}\left(\mathbf{0}, \sigma^{2} \mathbf{I}\right) .
\end{array}\right.
$$

No modelo não paramétrico, a relação funcional entre a variável resposta e a variável explicativa está em um espaço de funções muito amplo, ou seja, assumimos apenas que $f(\cdot)$ está em um espaço de funções sob determinadas restrições e busca-se uma combinação linear de funções desse espaço que melhor estime a variável de interesse. Dentre as diversas maneiras de escolher essa função, daremos ênfase à estimação via splines cúbicos.

Nessas primeiras seções, consideraremos que os valores da variável explicativa são distintos $(r=$ $n$ ), e abordaremos o caso de empates nas observações na Seção 2.11.

\subsection{Método de suavização spline cúbico}

Nos MANSs, é conveniente representar a função $f(\cdot)$ em (2.1) como um modelo linear, pois dessa forma é possível a utilização dos métodos usuais de estimação. Essa representação pode ser feita escolhendo uma função base e definindo o espaço de funções em que $f(\cdot)$ é um elemento. Um exemplo de função base é formado pelas bases polinomiais, em que a função $f(\cdot)$ é representada por

$$
f(t)=\sum_{j=1}^{s} \beta_{j} b_{j}(t)
$$


sendo $s$ o grau do polinômio, $b_{1}(t), \ldots, b_{s}(t)$ as funções base conhecidas e $\beta_{1}, \ldots, \beta_{s}$ os parâmetros desconhecidos. No entanto, quando é necessário um polinômio de grau muito alto para descrever a função $f(\cdot)$, é comum a ocorrência de erros na estimação. Como alternativa, essa função pode ser representada por um spline, que é uma curva composta por seções polinomiais de modo que juntas compõem uma função contínua.

Os splines são uma ferramenta do cálculo numérico que ganhou reconhecida atenção na área da Estatística pelo poder adaptativo na aproximação de funções. Nela, o domínio $[a, b]=\left[t_{1}, t_{n}\right]$ de $t$

é dividido em intervalos menores $\left[t_{1}, t_{2}\right], \ldots,\left[t_{k-1}, t_{k}\right]$, e polinômios são ajustados em cada intervalo $\left[t_{l}, t_{l+1}\right], l=1, \ldots, k-1$. Os $k$ pontos de união entre esses polinômios são denominados nós (knots), e o número de nós, bem como suas localizações, devem ser especificadas cuidadosamente.

De todas as configurações de polinômios possíveis, destacam-se os splines cúbicos (cubic splines), que são polinômios de terceiro grau e que, consequentemente, possuem primeiras e segundas derivadas contínuas nos nós. Os polinômios cúbicos possuem a vantagem de serem mais eficientes computacionalmente e, de acordo com Reinsch [1967] e Silverman [1984], possuem as seguintes propriedades:

1. $f(\cdot)$ é um polinômio cúbico em cada intervalo $\left[t_{l}, t_{l+1}\right]$;

2. para cada ponto $t_{l}$, a curva e suas duas primeiras derivadas são contínuas, embora sua terceira derivada possa ser descontínua.

Dentre os splines cúbicos, existem splines variantes segundo determinadas restrições. Por exemplo, podemos destacar os splines cúbicos naturais ( $\mathrm{SCN})$, que além das propriedades do spline cúbico, possuem uma propriedade adicional que, nos intervalos $\left.]-\infty, t_{1}\right]$ e $\left[t_{n},+\infty[\right.$ a segunda derivada de $f(\cdot)$ é zero, ou seja, $f(\cdot)$ é uma função linear a partir dos nós extremos. Para mais detalhes referentes aos splines, consultar De Boor [1978] ou Reinsch [1967].

\subsection{Critério de penalização}

Como o modelo (2.1) é ajustado numericamente por interpolação, a qualidade do ajuste pode não ser um bom critério de avaliação da curva $f(\cdot)$, uma vez que o ajuste será excelente quando interpolar todas observações da amostra. Nesse caso, teremos uma qualidade de ajuste ótima, porém uma curva pouco suave, ou seja, com variabilidade tão alta quanto a de $y$. Por outro lado, a priorização da suavidade da $f(\cdot)$ pode nos fazer perder informação no ajuste e não explicar o comportamento real dos dados. Uma solução para esse problema é adicionar um critério de penalização na soma de quadrados dos erros.

Na Seção 2.4.1 é apresentada a função de penalização introduzida por Handscomb [1966]. Na Seção 2.4.2 são definidas as adaptações das funções de verossimilhança e do logaritmo da função de verossimilhança, que são denominadas como função de verossimilhança penalizada e logaritmo da função de verossimilhança penalizada. Na Seção 2.4.3 temos as funções escore e informação de Fisher para os parâmetros do modelo (2.1), úteis no processo de estimação. 


\subsubsection{Função de penalização}

Considere o modelo proposto em (2.1). Um critério de avaliação da qualidade do ajuste pode ser dado pela minimização da soma dos quadrados dos erros sob a curva $f(\cdot)$, representada por

$$
\sum_{i=1}^{n}\left[y_{i}-f\left(t_{i}\right)\right]^{2}=(\mathbf{y}-\mathbf{N f})^{T}(\mathbf{y}-\mathbf{N f}),
$$

em que $\mathbf{y}$ é o vetor $(n \times 1)$ de valores observados da variável resposta, $\mathbf{N}$ é a matriz de incidência $(n \times n)$ e $\mathbf{f}$ é o vetor $(n \times 1)$ de parâmetros a serem estimados.

Como já mencionado, somente a avaliação da soma de quadrados dos erros não é suficiente para a avaliação da qualidade do ajuste. Handscomb [1966] introduziu um critério de penalização baseado na derivada em um grau menor da $f(\cdot)$ e avaliando sua integral em todo o domínio $[a, b]=\left[t_{1}, t_{n}\right]$ de $t$, de forma que quanto maior a curvatura da função, maior o valor da derivada avaliada e, consequentemente, maior o valor da integral. No caso dos splines cúbicos, a penalização é dada por

$$
\int_{a}^{b}\left[f^{\prime \prime}(t)\right]^{2} d t
$$

Logo, dentre todas as funções com primeira e segunda derivadas contínuas, deve-se encontrar a função $f(\cdot)$ que minimize a soma de quadrados dos erros penalizada

$$
\sum_{i=1}^{n}\left[y_{i}-f\left(t_{i}\right)\right]^{2}+\lambda \int_{a}^{b}\left[f^{\prime \prime}(t)\right]^{2} d t, \quad \lambda>0,
$$

em que $\lambda$ é o parâmetro de suavização que depende do número de nós escolhidos e de suas respectivas posições. Para $\lambda \rightarrow 0$, a segunda parcela de (2.6) tem pouca influência e prioriza-se o ajuste ótimo da curva, enquanto que para $\lambda \rightarrow \infty$ prioriza-se a suavidade da curva $f(\cdot)$, sob a restrição $f^{\prime \prime}(t)=0$ para a curva ser linear. A solução de (2.6) é única e, segundo Heckman [1986], corresponde ao spline cúbico natural $(\mathrm{SCN})$ com até $(n-2)$ nós internos $\left(t_{2}<\ldots<t_{n-1}\right)$, supondo uma amostra sem empates em $t$. Por ora, consideraremos $\lambda$ fixo, e discutiremos sua estimação na Seção 2.8 .

Para fins algébricos e computacionais, podemos escolher a representação do SCN de forma mais conveniente. Green e Silverman [1994] mostram que o termo da penalidade pode ser escrito na forma quadrática

$$
\int_{a}^{b}\left[f^{\prime \prime}(t)\right]^{2} d t=\mathbf{f}^{T} \mathbf{K f}
$$

em que $\mathbf{f}$ é o vetor $(n \times 1)$ de parâmetros a serem estimados e $\mathbf{K}$ é uma matriz $(n \times n)$ positiva definida que depende apenas dos nós, com estrutura dada por $\mathbf{K}=\mathbf{Q} \mathbf{R}^{-1} \mathbf{Q}^{T}$, em que $\mathbf{Q}$ é uma matriz tridiagonal $n \times(n-2)$ e $\mathbf{R}$ é uma matriz simétrica tridiagonal $(n-2) \times(n-2)$. Essa forma quadrática, no entanto, deve satisfazer o teorema dado a seguir.

Teorema 1. Seja $\gamma_{i}=f^{\prime \prime}\left(t_{i}\right)$ e $\gamma=\left(\gamma_{i}, \ldots, \gamma_{n}\right)^{T}$. Os vetores $\mathbf{f}$ e $\gamma$ especificam um $S C N$ se e somente se a condição

$$
\mathbf{Q}^{T} \mathbf{f}=\mathbf{R} \gamma
$$


é satisfeita. Se (2.8) é satisfeita, então a função penalidade definida em (2.7) é satisfeita.

A prova desse teorema encontra-se em Green e Silverman [1994]. Para a definição dos elementos das matrizes $\mathbf{Q}$ e $\mathbf{R}$, seja $h_{i}=t_{i+1}-t_{i}$, ou seja, a distância entre dois nós consecutivos $i$ e $i+1$. Os elementos $q_{i j}$ da matriz $\mathbf{Q}$ são definidos por

$$
q_{(j-1) j}=h_{j-1}^{-1}, \quad q_{j j}=-h_{j-1}^{-1}-h_{j}^{-1}, \quad q_{(j+1) j}=h_{j}^{-1}
$$

e $q_{i j}=0$ para $|i-j|>1, j=2, \ldots, n-1$. Já os elementos $r_{i j}$ de $\mathbf{R}$ são definidos por

$$
\begin{aligned}
r_{i i}=\frac{1}{3}\left(h_{i-1}+h_{i}\right), & i=1, \ldots, n-2, \\
r_{i(i+1)}=r_{i+1, i}=\frac{1}{6} h_{i}, & i=1, \ldots, n-1,
\end{aligned}
$$

e $r_{i j}=0$ para $|i-j|>1$.

Logo, o problema da minimização de (2.6) consiste em minimizar

$$
(\mathbf{y}-\mathbf{N f})^{T}(\mathbf{y}-\mathbf{N f})+\lambda \mathbf{f}^{T} \mathbf{K f}
$$

com relação à $\mathbf{f}$.

\subsubsection{Logaritmo da função de verossimilhança penalizada}

Seja $\boldsymbol{\theta}=\left(\mathbf{f}^{T}, \sigma^{2}\right)^{T}$ o vetor de parâmetros a ser estimado no modelo (2.1) definido no espaço $\Theta$, com $\Theta_{\mathbf{f}} \in \mathbb{R}$ e $\Theta_{\sigma^{2}} \in \mathbb{R}^{+}$. A função de verossimilhança do MANS é o produto das funções densidade de probabilidade da distribuição normal de parâmetros $\mu_{i}=f\left(t_{i}\right)$ e $\sigma^{2}$, dada por

$$
L(\boldsymbol{\theta})=\prod_{i=1}^{n} \frac{1}{\sqrt{2 \pi \sigma^{2}}} \exp \left\{-\frac{1}{2 \sigma^{2}}\left(y_{i}-f\left(t_{i}\right)\right)^{2}\right\} .
$$

Seja $\ell(\boldsymbol{\theta})$ o logaritmo da função de verossimilhança $L(\boldsymbol{\theta})$, que pode ser escrito como

$$
\ell(\boldsymbol{\theta})=-\frac{n}{2} \log 2 \pi \sigma^{2}-\frac{1}{2 \sigma^{2}} \sum_{i=1}^{n}\left[y_{i}-f\left(t_{i}\right)\right]^{2} .
$$

Os estimadores de máxima verossimilhança de $\mathbf{f}$ e $\sigma^{2}$ são os valores $\widehat{\mathbf{f}}$ e $\widehat{\sigma}$ que maximizam a função de verossimilhança (2.10) e, consequentemente, maximizam o logaritmo da função de verossimilhança (2.11). No entanto, Good e Gaskins [1971] mostram que a maximização dessa função não fornece uma boa estimação da curva $f(\cdot)$ e sugerem, assim como Handscomb [1966] o acréscimo de uma função de penalização definida como $\frac{1}{2} \lambda^{*} \int\left[f^{\prime \prime}(t)\right]^{2} d t$, em que $\lambda^{*}$ é uma função do parâmetro de suavização $\lambda$ definido na Seção 2.4.1. Logo, o logaritmo da função de verossimilhança penalizada pode ser escrito como

$$
\ell_{p}(\boldsymbol{\theta}, \lambda)=-\frac{n}{2} \log 2 \pi \sigma^{2}-\frac{1}{2 \sigma^{2}} \sum_{i=1}^{n}\left[y_{i}-f\left(t_{i}\right)\right]^{2}-\frac{1}{2} \lambda^{*} \int\left[f^{\prime \prime}(t)\right]^{2} d t
$$


Para $\lambda^{*}=\lambda / \sigma^{2}$, a maximização de $\ell_{p}(\boldsymbol{\theta}, \lambda)$ é equivalente à minimização de (2.6). Para o estudo da estimação dos parâmetros do vetor $\boldsymbol{\theta}$ nas próximas seções, o parâmetro de suavização $\lambda$ será considerado fixo, e posteriormente estudado na Seção 2.8 .

\subsubsection{Função escore e informação de Fisher penalizada}

Matricialmente, o logaritmo da função de verossimilhanca penalizada (2.12) pode ser expresso como

$$
\ell_{p}(\boldsymbol{\theta}, \lambda)=-\frac{1}{2 \sigma^{2}}(\mathbf{y}-\mathbf{N f})^{T}(\mathbf{y}-\mathbf{N f})-\frac{1}{2} \lambda \mathbf{f}^{T} \mathbf{K f}
$$

Considere $\lambda$ fixo e seja $\mathbf{U}_{p}(\boldsymbol{\theta})$ o vetor $(n+1 \times 1)$ da função escore penalizada

$$
\mathbf{U}_{p}(\boldsymbol{\theta})=\left(\begin{array}{c}
\mathbf{U}_{p}^{\mathbf{f}}(\boldsymbol{\theta}) \\
\mathbf{U}_{p}^{\sigma^{2}}(\boldsymbol{\theta})
\end{array}\right),
$$

em que $\mathbf{U}_{p}^{\mathrm{f}}$ e $\mathrm{U}_{p}^{\sigma^{2}}$ são, respectivamente, o vetor $(n \times 1)$ das funções escore associadas ao efeito não paramétrico e a função escore associada à variância, definidas por

$$
\begin{aligned}
\mathbf{U}_{p}^{\mathbf{f}}(\boldsymbol{\theta}) & =\frac{\partial \ell_{p}(\boldsymbol{\theta})}{\partial \mathbf{f}} \quad \mathrm{e} \\
\mathrm{U}_{p}^{\sigma^{2}}(\boldsymbol{\theta}) & =\frac{\partial \ell_{p}(\boldsymbol{\theta})}{\partial \sigma^{2}} .
\end{aligned}
$$

Após algumas manipulações algébricas, não é difícil verificar que

$$
\begin{aligned}
\mathbf{U}_{p}^{\mathbf{f}}(\boldsymbol{\theta}) & =\sigma^{-2} \mathbf{N}^{T}(\mathbf{y}-\mathbf{N f})-\lambda \mathbf{K f} \\
\mathrm{U}_{p}^{\sigma^{2}}(\boldsymbol{\theta}) & =\frac{1}{2 \sigma^{2}}\left[\frac{(\mathbf{y}-\mathbf{N f})^{T}(\mathbf{y}-\mathbf{N f})}{\sigma^{2}}-n\right] .
\end{aligned}
$$

Sob condições de regularidade, podemos definir a matriz bloco diagonal de informação de Fisher penalizada $(n+1 \times n+1)$ como

$$
\mathcal{I}_{p}(\boldsymbol{\theta})=\left(\begin{array}{cc}
\mathcal{I}_{p}^{\mathbf{f}}(\boldsymbol{\theta}) & \mathbf{0} \\
\mathbf{0} & \mathcal{I}_{p}^{\sigma^{2}}(\boldsymbol{\theta})
\end{array}\right)
$$

em que $\mathcal{I}_{p}^{\mathbf{f}}$ e $\mathcal{I}_{p}^{\sigma^{2}}$ são, respectivamente, a matriz de informação de Fisher de ordem $(n \times n)$ dos parâmetros de $\mathbf{f}$ e a informação de Fisher de $\sigma^{2}$, calculadas a partir do valor negativo da esperança das segundas derivadas com respeito, respectivamente, ao efeito não paramétrico e à variância, ou seja

$$
\begin{aligned}
\mathcal{I}_{p}^{\mathbf{f}}(\boldsymbol{\theta}) & =-E\left(\frac{\partial^{2} \ell_{p}(\boldsymbol{\theta})}{\partial \mathbf{f} \partial \mathbf{f}^{T}}\right)=\sigma^{-2} \mathbf{N}^{T} \mathbf{N}+\lambda \mathbf{K} \quad \mathrm{e} \\
\mathcal{I}_{p}^{\sigma^{2}}(\boldsymbol{\theta}) & =-E\left(\frac{\partial^{2} \ell_{p}(\boldsymbol{\theta})}{\partial \sigma^{4}}\right)=\frac{n}{2 \sigma^{4}} .
\end{aligned}
$$


Detalhes sobre o cálculo das primeiras e segundas derivadas do logaritmo da função de verossimilhança penalizada em relação à f e $\sigma^{2}$ encontram-se no Apêndice B.

\subsection{Estimação dos parâmetros}

Nos casos em que as condições de regularidade estão satisfeitas, os estimadores de máxima verossimilhança penalizados de $\mathbf{f}$ e $\sigma^{2}$ podem ser obtidos como soluções das equações $\mathbf{U}_{p}^{\mathbf{f}}(\boldsymbol{\theta})=\mathbf{0}$ e $U_{p}^{\sigma^{2}}(\boldsymbol{\theta})=0$. Para a obtenção do estimador $\mathbf{f}$, não é necessário conhecermos $\sigma^{2}$, porém a estimação do parâmetro de dispersão depende do vetor f , e assim podemos obter uma solução de $\sigma^{2}$ como função de $\mathbf{f}$ através da verossimilhança penalizada perfilada.

Na Seção 2.5.1 obtemos o estimador $\widehat{\mathbf{f}}$ e definimos a matriz de suavização e a matriz hat, além de alguns resultados referentes a esses estimadores; na Seção 2.5.2 definimos os graus de liberdade efetivos (GLE) e, por fim, na Seção 2.5.3 obtemos o estimador de $\sigma^{2}$ em função de $\widehat{\mathbf{f}}$.

\subsubsection{Estimação da função não paramétrica}

Supondo que o logaritmo da função de verossimilhança penalizada $\ell_{p}(\boldsymbol{\theta}, \lambda)$, definido em (2.12), é uma função que satisfaz certas condições de regularidade, o valor de $\mathbf{f}$ que maximiza $\ell_{p}(\boldsymbol{\theta}, \lambda)$ é chamado de estimador de máxima verossimilhança penalizado (EMVP) de $\mathbf{f}$, denotado por $\widehat{\mathbf{f}}$, e satisfaz a desigualdade

$$
\ell_{p}(\widehat{\mathbf{f}}, \lambda) \geq \sup _{\mathbf{f} \in \Theta_{\mathbf{f}}} \ell_{p}(\mathbf{f}, \lambda)
$$

Nesse caso, como a função $\ell_{p}(\boldsymbol{\theta}, \lambda)$ depende de $f(\cdot)$ através da avaliação funcional $f\left(t_{i}\right)$, e $\mathbf{f}$ e $\mathbf{K}$ são avaliados em $\mathbf{t}=\left(t_{1}, \ldots, t_{n}\right)^{T}$ de dimensão finita, temos que, sob certas condições de regularidade, o estimador de máxima verossimilhança penalizado de $f(\cdot)$ do modelo (2.1) existe e é único, e corresponde a um SCN que é completamente determinada pelo vetor finito dimensional t , ou seja, admissível em um espaço finito.

Considere $\lambda$ fixo. Para o estimador de máxima verossimilhança $\widehat{\mathbf{f}}$,

$$
\mathbf{U}_{p}^{\mathbf{f}}(\boldsymbol{\theta})=\frac{\mathbf{N}^{T}(\mathbf{y}-\mathbf{N f})-\lambda \mathbf{K} \mathbf{f}}{\sigma^{2}}=\mathbf{0}
$$

e, após manipulações algébricas, chega-se ao resultado

$$
\widehat{\mathbf{f}}=\left(\mathbf{N}^{T} \mathbf{N}+\lambda \mathbf{K}\right)^{-1} \mathbf{N}^{T} \mathbf{y}=\mathbf{S}(\lambda) \mathbf{y}
$$

em que $\mathbf{S}(\lambda)$ é uma matriz $(n \times n)$ denominada matriz de suavização que depende do parâmetro de suavização $\lambda$. De fato, é possível verificar que $\widehat{\mathbf{f}}$ é o EMVP de f, já que a matriz de segundas derivadas do logaritmo da função de verossimilhança penalizada é negativa definida (vide matriz no Apêndice B). Consequentemente, por (2.18) o vetor de valores ajustados $\widehat{\mathbf{y}}=\left(\widehat{y}_{1}, \ldots, \widehat{y}_{n}\right)^{T}$ pode ser 
escrito na forma

$$
\widehat{\mathbf{y}}=\mathbf{N} \widehat{\mathbf{f}}=\mathbf{N}\left(\mathbf{N}^{T} \mathbf{N}+\lambda \mathbf{K}\right)^{-1} \mathbf{N}^{T} \mathbf{y}=\mathbf{H}(\lambda) \mathbf{y}
$$

em que $\widehat{\mathbf{y}}$ é a combinação linear das observações da variável resposta $\mathbf{y}$, com coeficientes dados pelos elementos da matriz $\mathbf{H}(\lambda)(n \times n)$ simétrica denominada matriz hat ou projetora. Assim como no modelo de regressão usual, a matriz $\mathbf{H}(\lambda)$ é importante na predição e na análise de diagnóstico através da detecção de pontos influentes, porém não possui a propriedade de idempotência $\left(\mathbf{H}(\lambda)^{2} \neq\right.$ $\mathbf{H}(\lambda)$ ), ou seja, nem sempre as propriedades da matriz hat do modelo linear usual são aplicáveis no MANS.

\subsubsection{Graus de liberdade}

Vamos supor o ajuste da função $f(\cdot)$ pelo modelo linear usual de uma amostra de $n$ observações, assumindo que $f(t)$ é da forma $\sum_{j=1}^{s} \beta_{j} b_{j}(t), \operatorname{com} s=2, b_{1}(t)=1$ e $b_{2}(t)=t$ no modelo linear simples. Nesse caso, a matriz hat do modelo linear usual, digamos $\mathcal{H}$, é uma matriz $(n \times n)$ tal que

$$
\widehat{\mathbf{y}}=\mathcal{H} \mathbf{y}
$$

com espaço de dimensão $s, s=\operatorname{tr}\{\mathcal{H}\}$ o número de parâmetros a serem estimados no modelo e os graus de liberdade associados à regressão. Dessa forma, os graus de liberdade associados aos resíduos ficam dados por $n-s=\operatorname{tr}\{\mathbf{I}-\mathcal{H}\}$.

Nos MANSs, a matriz $\mathbf{H}(\lambda)$ definida em (2.19) desempenha papel semelhante ao da matrix hat do modelo linear usual. Essa característica motivou o cálculo do número de graus de liberdade efetivos (GLE), que dá uma indicação do número efetivo de parâmetros considerados no modelo não paramétrico. Dessa forma, define-se os GLE associados à regressão como

$$
\mathrm{GLE}=\operatorname{tr}\{\mathbf{H}(\lambda)\}
$$

Como o cálculo do GLE depende do parâmetro de suavização $\lambda$, se supormos $\lambda \rightarrow 0$, estaremos priorizando o ajuste do modelo com a interpolação das $n$ observações, ou seja, GLE $\rightarrow n$. Porém, quando $\lambda \rightarrow \infty$, nosso modelo será equivalente ao modelo de regressão linear simples usual, ou seja, GLE $=2$. Nesse caso, temos que o domínio de GLE é o intervalo $[2 ; n]$.

Sendo $\mathbf{H}(\lambda)$ simétrica com autovalores $\zeta_{1}, \ldots, \zeta_{n}$, temos a relação imediata

$$
\operatorname{tr}\{\mathbf{H}(\lambda)\}=\sum_{i=1}^{n} \zeta_{i}
$$

Existem ainda pelo menos duas outras maneiras de definir os graus de liberdade de suavizadores lineares, porém o número de graus de liberdade definido como sendo o traço da matriz suavizadora é computacionalmente mais simples de ser calculado. Para detalhes sobre as demais definições de graus de liberdade ver, por exemplo, Buja et al. [1989]. 


\subsubsection{Estimação do parâmetro de dispersão}

Para a estimação do parâmetro $\sigma^{2}$, considere o EMVP $\widehat{\mathbf{f}}$ obtido em (2.18). Igualando a função escore parcial $U_{p}^{\sigma^{2}}$ a zero, obtém-se o estimador

$$
\widehat{\sigma}^{2}=\frac{(\mathbf{y}-\mathbf{N} \widehat{\mathbf{f}})^{T}(\mathbf{y}-\mathbf{N} \widehat{\mathbf{f}})}{n}
$$

No entanto, o estimador (2.23) é viesado e, nesse caso, o mais apropriado é utilizarmos o estimador amostral $s^{2}$, dado pela razão entre a soma dos quadrados dos resíduos e o GLE associado aos resíduos, ou seja,

$$
s^{2}=\frac{(\mathbf{y}-\mathbf{N} \widehat{\mathbf{f}})^{T}(\mathbf{y}-\mathbf{N} \widehat{\mathbf{f}})}{\operatorname{tr}\{\mathbf{I}-\mathbf{H}(\lambda)\}}
$$

Para maiores detalhes referente à $U_{p}^{\sigma^{2}}$, ver Apêndice B.

\subsection{Inferências para $\mathrm{f}$}

Nesta seção trataremos de estudar a distribuição do estimador $\widehat{\mathbf{f}}$, testes de hipóteses e erro padrão para os MANs. Na Seção 2.6.1, veremos que o estimador $\widehat{\mathbf{f}}$ é viesado para f, porém assintoticamente não viesado. Já na Seção 2.6.2 trataremos da obtenção da matriz de variância-covariância através da inversa da informação de Fisher para f, já que Wahba [1983] mostra sob o enfoque bayesiano que se definirmos uma distribuição a priori para $\mathbf{f}$ e utilizarmos a distribuição a posteriori $\mathbf{f} \mid \mathbf{y}$ para fazer inferência, a matriz de variância-covariância dessa posteriori coincide com a inversa da informação de Fisher para f. E por fim, trataremos da Seção 2.6.3 os possíveis testes para a hipótese $\mathbf{f}=\mathbf{0}$.

\subsubsection{Distribuição de $\widehat{\mathbf{f}}$}

Por definição, o estimador $\widehat{\mathbf{f}}$ é dito não viesado para $\mathbf{f}$ se, para todo $\widehat{\mathbf{f}} \in \Theta_{\mathbf{f}}, \mathrm{E}(\widehat{\mathbf{f}})=\mathbf{f}$. Considerando o estimador obtido em (2.18), temos

$$
\mathrm{E}(\widehat{\mathbf{f}})=\left(\mathbf{N}^{T} \mathbf{N}+\lambda \mathbf{K}\right)^{-1} \mathbf{N}^{T} \mathbf{N} \mathbf{f} \neq \mathbf{f} .
$$

No entanto, segundo Zhang et al. [1998], $\widehat{\mathbf{f}}$ é um estimador assintoticamente não viesado de f, já que à medida que aumentamos o tamanho da amostra para a estimação de $f(\cdot)$, priorizamos o ajuste sem levar em consideração a suavidade da curva, ou seja, $\lambda \downarrow 0$ e, portanto, $E(\widehat{\mathbf{f}}) \stackrel{\lambda \downarrow 0}{\longrightarrow} \mathbf{f}$. Além disso, quando $\mathbf{f}=\mathbf{0}$, temos que $\mathrm{E}(\widehat{\mathbf{f}})=\mathbf{0}$, e esse resultado pode ser bastante útil para realizarmos testes de hipóteses para esse tipo de modelo, sobretudo $\mathbf{f}=\mathbf{0}$.

Já a matriz de variância-covariância de $\widehat{\mathbf{f}}$ pode ser escrita como

$$
\operatorname{Var}(\widehat{\mathbf{f}})=\left(\mathbf{N}^{T} \mathbf{N}+\lambda \mathbf{K}\right)^{-1} \mathbf{N}^{T} \mathbf{N}\left(\mathbf{N}^{T} \mathbf{N}+\lambda \mathbf{K}\right)^{-1} \sigma^{2}=\mathbf{V}_{\mathbf{f}}
$$

Para amostras grandes, ou dado que y tem distribuição Normal, podemos concluir que a dis- 
tribuição de $\widehat{\mathbf{f}}$ é aproximadamente $\widehat{\mathbf{f}} \sim \mathcal{N}_{r}\left(\mathrm{E}(\widehat{\mathbf{f}}), \mathbf{V}_{\mathbf{f}}\right)$. É importante observar que a matriz $\mathbf{V}_{\mathbf{f}}$ pode não ter posto completo, e nesse caso $\widehat{\mathbf{f}} \sim \mathcal{N}_{r^{*}}\left(\mathrm{E}(\widehat{\mathbf{f}}), \mathbf{V}_{\mathbf{f}}^{-}\right)$, em que $r^{*}$ é o posto de $\mathbf{V}_{\mathbf{f}}$ e $\mathbf{V}_{\mathbf{f}}^{-}$é a inversa generalizada (ver, por exemplo, Wood [2006]). Assim, a utilização da inversa da matriz de informação de Fisher pode ser mais conveniente como discutiremos na próxima seção.

\subsubsection{Erro padrão}

Sabemos que nos modelos lineares usuais as matrizes de variância-covariância assintóticas das estimativas de máxima verossimilhança são obtidas pela inversa da matriz de informação observada ou esperada. Nos modelos não paramétricos, vários autores já discutiram esse problema no contexto de estimação via máxima verossimilhança penalizada. No caso do MANS, Wahba [1983] propõe estimar a matriz de variância-covariância sob enfoque bayesiano e mostra que a matriz de variânciacovariância corresponde à inversa da matriz de informação de Fisher calculada a partir da função de verossimilhança penalizada. Sendo assim, seja $g(\mathbf{f})$ uma distribução à priori (geralmente imprópria) de $\mathbf{f}$

$$
g(\mathbf{f}) \propto \exp \left\{-\frac{1}{2 \tau} \mathbf{f}^{T} \mathbf{K f}\right\}
$$

em que $\tau$ é o parâmetro que controla a dispersão da priori. Sabemos também que a função densidade de probabilidade de $\mathbf{y}$ dado $\mathbf{f}$ é proporcional a

$$
g(\mathbf{y} \mid \mathbf{f}) \propto \exp \left\{-\frac{1}{2 \sigma^{2}}(\mathbf{y}-\mathbf{N f})^{T}(\mathbf{y}-\mathbf{N f})\right\} .
$$

Por Bayes, temos então que a distribuição a posteriori $f(\mathbf{f} \mid \mathbf{y})$ é proporcional a

$$
\begin{aligned}
g(\mathbf{f} \mid \mathbf{y}) & \propto \exp \left\{-\frac{1}{2 \sigma^{2}}(\mathbf{y}-\mathbf{N f})^{T}(\mathbf{y}-\mathbf{N f})+\frac{1}{2 \tau} \mathbf{f}^{T} \mathbf{K} \mathbf{f}\right\} \\
& \propto \exp \left\{-\frac{1}{2}\left[-\frac{2 \mathbf{f}^{T} \mathbf{N}^{T} \mathbf{y}}{\sigma^{2}}+\mathbf{f}^{T}\left(\frac{\mathbf{N}^{T} \mathbf{N}}{\sigma^{2}}+\frac{\mathbf{K}}{\tau}\right)\right] \mathbf{f}\right\} .
\end{aligned}
$$

Para descobrirmos a distribuição de $g(\mathbf{f} \mid \mathbf{y})$, suponha $\boldsymbol{\alpha}$ uma variável aleatória de distribuição $\boldsymbol{\alpha} \sim \mathcal{N}\left(\left(\mathbf{N}^{T} \mathbf{N}+\lambda \mathbf{K}\right)^{-1} \mathbf{N}^{T} \mathbf{y},\left(\mathbf{N}^{T} \mathbf{N}+\lambda \mathbf{K}\right)^{-1} \sigma^{2}\right)$. A função densidade de probabilidade $g(\boldsymbol{\alpha})$ é proporcional a

$$
g(\boldsymbol{\alpha}) \propto \exp \left\{-\frac{1}{2}\left[-\frac{2 \boldsymbol{\alpha}^{T} \mathbf{N}^{T} \mathbf{y}}{\sigma^{2}}+\boldsymbol{\alpha}^{T}\left(\frac{\mathbf{N}^{T} \mathbf{N}}{\sigma^{2}}+\frac{\lambda \mathbf{K}}{\sigma^{2}}\right)\right] \boldsymbol{\alpha}\right\}
$$

Comparando (2.29) e (2.30), fica evidente que, para $\tau=\sigma^{2} / \lambda$, temos que

$$
\mathbf{f} \mid \mathbf{y} \sim \mathcal{N}_{r}\left(\left(\mathbf{N}^{T} \mathbf{N}+\lambda \mathbf{K}\right)^{-1} \mathbf{N}^{T} \mathbf{y},\left(\mathbf{N}^{T} \mathbf{N}+\lambda \mathbf{K}\right)^{-1} \sigma^{2}\right) \sim \mathcal{N}_{r}\left(\widehat{\mathbf{f}},\left(\mathbf{N}^{T} \mathbf{N}+\lambda \mathbf{K}\right)^{-1} \sigma^{2}\right)
$$

em que a variância de $\boldsymbol{\beta}$ coincide com a inversa da matriz de informação de Fisher em (2.16). Esse resultado permite uma base consistente para estimarmos as matrizes de variância-covariância assintóticas dos estimadores baseadas na inversa da matriz de informação de Fisher penalizada 
$\mathcal{I}_{p}^{-1}(\boldsymbol{\theta})$. Portanto, temos que

$$
\widehat{\operatorname{Var}(\widehat{\boldsymbol{\theta}})} \approx \mathcal{I}_{p}^{-1}(\widehat{\boldsymbol{\theta}})
$$

com

$$
\mathcal{I}_{p}^{-1}(\boldsymbol{\theta})=\left(\begin{array}{cc}
\mathcal{I}_{p}^{\mathbf{f}^{-1}}(\boldsymbol{\theta}) & \mathbf{0} \\
\mathbf{0} & \mathcal{I}_{p}^{{\sigma^{2}}^{-1}}(\boldsymbol{\theta})
\end{array}\right)
$$

em que

$$
\widehat{\operatorname{Var}(\mathbf{f})} \approx \mathcal{I}_{p}^{\mathbf{f}^{-1}}(\boldsymbol{\theta})=\left[\sigma^{-2} \mathbf{N}^{T} \mathbf{N}+\lambda \mathbf{K}\right]^{-1}
$$

Para um estudo mais detalhado do enfoque bayesiano como justificativa para usarmos a inversa da informação de Fisher para estimarmos os erros padrão, detalhes sobre estrutura da função à priori e extensões para a família exponencial, consultar Wahba [1983] e Wood [2006].

\subsubsection{Testes de hipóteses}

Se tratarmos o logaritmo da função de verossimilhança penalizada como uma verossimilhança usual, podemos utilizar os testes de hipóteses já conhecidos na literatura. Por exemplo, para variâncias conhecidas podemos aplicar o teste da razão de verossimilhanças ou teste de Wald através da inversa da matriz de informação de Fisher para fazer inferência para $n$ grande a respeito de $\mathbf{f}$ e supondo normalidade assintótica de $\widehat{\mathbf{f}}$.

Vamos considerar as hipóteses $H_{0}: \mathbf{f}=\mathbf{0}$ contra $H_{1}: \mathbf{f} \neq \mathbf{0}$. Os testes de razão de verossimilhanças e de Wald são, respectivamente, dados por

$$
\xi_{R V}=\widehat{\mathbf{f}}^{T} \mathbf{N}^{T}(2 \mathbf{y}-\mathbf{N} \widehat{\mathbf{f}})
$$

e

$$
\xi_{W}=\widehat{\mathbf{f}}^{T} \mathbf{V}_{\mathbf{f}}^{-1} \widehat{\mathbf{f}}
$$

que, sob a hipótese nula, $\xi_{R V}$ e $\xi_{W} \sim \chi_{r}^{2}$. No caso de $\mathbf{V}_{\mathbf{f}}$ não ser de posto completo, a inversa de $\mathbf{V}_{\mathbf{f}}$ pode ser substituída por uma inversa generalizada $\mathbf{V}_{\mathbf{f}}^{-}$.

Para variâncias desconhecidas, o teste utilizado é o teste $\mathrm{F}$, nesse caso dado por

$$
\mathrm{F}=\frac{\widehat{\mathbf{f}}^{T} \mathbf{V}_{\mathbf{f}}^{-1} \widehat{\mathbf{f}} / n}{s^{2} /(n-\mathrm{GLE})},
$$

que sob a hipótese nula segue distribuição $F_{n, \text { GLE }}$.

Segundo Wood [2006], quando o parâmetro de suavização é desconhecido as estatísticas do teste sob a hipótese nula são geralmente menores que deveriam ser, e a hipótese nula acaba não sendo rejeitada muito facilmente. Em termos práticos, se essas estatísticas estiverem em torno de um limite para a aceitação ou rejeição de uma hipótese, elas devem ser analisadas com cautela. Porém, essas 
estatísticas ainda são preferidas aos testes que não consideram o critério de penalização.

\subsection{Técnicas de diagnóstico}

No processo de ajuste de qualquer modelo de regressão, a análise de diagnóstico é uma etapa importante na verificação das suposições feitas para o modelo e da existência de observações com alguma influência desproporcional nos resultados do ajuste.

Nesta seção, estudaremos como a matriz hat pode ser utilizada na análise de diagnóstico, mesmo não tendo a propriedade de idempotência da matriz hat dos modelos normais usuais. Apresentaremos as técnicas de diagnóstico desenvolvidas, como pontos de alavanca, pontos aberrantes e distância de Cook, além da implementação gráfica dessas medidas no software R para modelos ajustados via pacote gamlss.

\subsubsection{Propriedades da matriz hat}

Seja $\mathcal{H}$ a matriz hat do modelo linear usual definida na Seção 2.5.2. Podemos ver, por exemplo, em Neter et al. [1996], que a matriz $\mathcal{H}$ de elementos $\mathcal{H}=\left\{h_{i j}\right\}$ tem as propriedades de simetria e idempotência, e tem papel importante na predição já que, por (2.20), cada elemento $h_{i j}$ da matriz $\mathcal{H}$ nos diz a influência de $y_{j}$ na predição de $\widehat{y}_{i}$ e, em particular, os elementos da diagonal principal $h_{i i}$ indicam qual a influência $y_{i}$ tem em sua própria predição.

Além disso, a matriz $\mathcal{H}$ também tem papel importante no diagnóstico dos resíduos. Seja $\mathbf{r}=$ $\left(r_{1}, \ldots, r_{n}\right)^{T}$ o vetor de resíduos ordinários de elementos $r_{i}=y_{i}-\widehat{y}_{i}$, que mede a discrepância entre o valor observado e o esperado da $i$-ésima observação. Logo,

$$
\mathbf{r}=\mathbf{y}-\mathcal{H} \mathbf{y}=(\mathbf{I}-\mathcal{H}) \mathbf{y}
$$

No caso do MANS com estimação via splines, também existe a relação linear entre o vetor de valores ajustados $\widehat{\mathbf{y}}$ e o vetor de valores observados $\mathbf{y}$ através da matriz $\mathbf{H}(\lambda)$ definida em (2.19), dada por

$$
\widehat{\mathbf{y}}=\mathbf{H}(\lambda) \mathbf{y}
$$

ou seja, cada elemento $h_{i j}(\lambda)$ da matriz $\mathbf{H}$ nos diz a influência de $y_{j}$ na predição de $\widehat{y}_{i}$.

Análogo à (2.32), definimos $\mathbf{r}=\left(r_{1}, \ldots, r_{n}\right)^{T}$ o vetor de resíduos ordinários do MANS de elemento $r_{i}=y_{i}-\widehat{y}_{i}$, tal que

$$
\mathbf{r}=\mathbf{y}-\mathbf{N} \widehat{\mathbf{f}}=\mathbf{y}-\mathbf{H}(\lambda) \mathbf{y}=(\mathbf{I}-\mathbf{H}(\lambda)) \mathbf{y}
$$

A matriz $\mathbf{H}(\lambda)$ é simétrica, porém não possui a propriedade de idempotência, ou seja, nem sempre as propriedades e resultados da matriz hat do modelo linear usual podem ser aplicadas no caso dos modelos não paramétricos. No entanto, Eubank [1984] propõe uma aproximação da matriz $\mathbf{H}(\lambda)$ para uma matriz $\mathbf{H}^{*}=\left\{h_{i j}^{*}\right\}$ idempotente e deriva várias propriedades básicas também presentes 
na matriz hat do modelo linear usual, tornando possível a utilização da matriz $\mathbf{H}(\lambda)$ na análise de diagnóstico no MANS.

Utilizando estudos de Wahba [1978], Eubank define uma matriz $\mathbf{T}(n \times m)$, com $m$ dado pela ordem do polinômio utilizado para o ajuste da variável não paramétrica, e cada elemento $\phi_{i j}(t)$, $i=1, \ldots, n$ e $j=1, \ldots, m$ dado por

$$
\phi_{i j}(t)=\frac{t_{i}^{j-1}}{(j-1) !} .
$$

No nosso caso, temos $m=2 \mathrm{e}$, portanto, temos que a matriz $\mathbf{T}$ é equivalente à matriz de planejamento $\mathbf{X}$ do modelo linear usual com intercepto. Sendo assim, podemos definir a matriz $\mathbf{H}^{*}$ como

$$
\mathbf{H}^{*}=\mathbf{T}\left(\mathbf{T}^{T} \mathbf{T}\right)^{-1} \mathbf{T}^{T}
$$

ou seja, $\mathbf{H}^{*}$ é equivalente à matriz simétrica e idempotente $\mathcal{H}$ do modelo linear usual e consequentemente, $\mathbf{I}-\mathbf{H}^{*}$ também é idempotente e pode ser escrita como

$$
\mathbf{I}-\mathbf{H}^{*}=\mathbf{U U}^{T}
$$

com $\mathbf{U}$ sendo uma matriz $n \times(n-2)$ que satisfaz $\mathbf{U}^{T} \mathbf{T}=\mathbf{0}$ e $\mathbf{U}^{T} \mathbf{U}=\mathbf{I}_{(n-2) \times(n-2)}$. Eubank define também uma "covariância de Kernel"

$$
\mathcal{Q}(s, t)=\int_{a}^{s}(s-u)(t-u) d u, \quad s \leq t,
$$

e a matriz $\mathcal{Q}_{(n \times n)}$ com entradas $\mathcal{Q}\left(t_{i}, t_{j}\right)=\mathcal{Q}\left(t_{j}, t_{i}\right)$, ou seja, $\mathcal{Q}$ é uma matriz de covariâncias simétrica. Consequentemente, o produto $\mathbf{U}^{T} \mathcal{Q} \mathbf{U}$ é uma matriz simétrica e, segundo Eubank, positiva definida, ou seja, todos os autovalores $d_{1}, d_{2}, \ldots, d_{n-2}$ da matriz $\mathbf{U}^{T} \mathcal{Q} \mathbf{U}$ são maiores do que zero. Se $\boldsymbol{\Gamma}$ é a matriz $(n-2 \times n-2)$ dos autovetores normalizados de $\mathbf{U}^{T} \mathcal{Q} \mathbf{U}$, temos que $\boldsymbol{\Gamma} \boldsymbol{\Gamma}^{T}=\boldsymbol{\Gamma}^{T} \boldsymbol{\Gamma}=\mathbf{I}$, e a matriz $\mathbf{H}(\lambda)$ pode ser expressa tal que

$$
\mathbf{I}-\mathbf{H}(\lambda)=\mathbf{U} \boldsymbol{\Gamma D}(\lambda) \boldsymbol{\Gamma}^{T} \mathbf{U}^{T}
$$

em que $\mathbf{D}(\lambda)=\operatorname{diag}\left(\left(1+d_{1} / n \lambda\right)^{-1}, \ldots,\left(1+d_{n-2} / n \lambda\right)^{-1}\right)$. Essas são as premissas para Eubank enunciar o seguinte teorema.

Teorema 2. A matriz $\mathbf{H}(\lambda)$ satisfaz

1. $0 \leq h_{i i}(\lambda) \leq 1$,

2. $-1 \leq h_{i j}(\lambda) \leq 1$, para $i \neq j$,

3. $h_{i i}(\lambda)=1$ se e somente se $h_{i j}(\lambda)=0$ para todo $i \neq j$ e

4. $\sum_{i=1}^{n} h_{i j}(\lambda)=1$. 
Essas propriedades estão fortemente relacionadas com $\mathbf{H}^{*}$, já que $h_{i i}(\lambda) \downarrow h_{i i}^{*}$ para $\lambda \rightarrow \infty$ se $h_{i i}^{*} \neq 1$. Além disso, $h_{i j}(\lambda) \rightarrow h_{i j}^{*}$ para $\lambda \rightarrow \infty$ e para $\lambda$ suficientemente grande com $h_{i j}^{*} \neq 0, h_{i j}(\lambda)$ e $h_{i j}^{*}$ possuem o mesmo sinal. Por outro lado, se $\lambda \rightarrow 0$ então $h_{i j}(\lambda) \rightarrow 0$ e dado que $h_{i i}^{*} \neq 1, h_{i i}(\lambda) \uparrow 1$.

A prova detalhada de todos os itens encontram-se em Eubank [1984]. Porém, seja $\mathbf{B}=\mathbf{U} \boldsymbol{\Gamma}$ e observe que $\mathbf{B B}^{T}=\mathbf{U} \boldsymbol{\Gamma} \boldsymbol{\Gamma}^{T} \mathbf{U}^{T}=\mathbf{U U}^{T}=\mathbf{I}-\mathbf{H}^{*}$, ou seja,

$$
1-h_{i i}^{*}=\sum_{j=1}^{n-2} b_{i j}^{2}=\sum_{j=1}^{n-2} u_{i j}^{2}
$$

De (2.35), temos que

$$
1-h_{i i}(\lambda)=\sum_{j=1}^{n-2} b_{i j}^{2}\left(1+d_{j} / n \lambda\right)^{-1}
$$

e como $d_{j}, n$ e $\lambda$ são maiores do que zero, $0<\left(1+d_{j} / n \lambda\right)^{-1} \leq 1$. Dessa expressão e de (2.36), segue que

$$
0 \leq h_{i i}(\lambda) \leq 1-h_{i i}^{*} \Rightarrow h_{i i}^{*} \leq h_{i i}(\lambda) \Rightarrow 0 \leq h_{i i}^{*} \leq h_{i i}(\lambda) \Rightarrow 0 \leq h_{i i}(\lambda) \leq 1 .
$$

De maneira análoga,

$$
\begin{gathered}
h_{i j}^{*}=-\sum_{k=1}^{n-2} b_{i k} b_{j k} \quad \mathrm{e} \\
h_{i j}(\lambda)=-\sum_{k=1}^{n-2} b_{i k} b_{j k}\left(1+d_{k} / n \lambda\right)^{-1}
\end{gathered}
$$

e aplicando a desigualdade de Cauchy-Schwarz, temos que

$$
\left|h_{i j}(\lambda)\right| \leq \max _{1 \leq l \leq n}\left(1-h_{l l}(\lambda)\right) \leq 1
$$

em que $1-h_{l l}(\lambda) \geq 0$ por $(2.38)$, e portanto $0 \leq h_{i j}(\lambda) \leq 1$.

Além disso, se $h_{i i}^{*} \neq 1, b_{i j} \neq 0$ para pelo menos um $j$, segue que

$$
\begin{gathered}
\lim _{\lambda \rightarrow \infty} h_{i i}(\lambda)=\sum_{j=1}^{n-2} b_{i j}^{2}=\sum_{j=1}^{n-2} u_{i j}^{2}=h_{i i}^{*}, \\
\lim _{\lambda \rightarrow \infty} h_{i j}(\lambda)=-\sum_{k=1}^{n-2} b_{i k} b_{j k}=-\sum_{j=1}^{n-2} u_{i k} u_{j k}=h_{i j}^{*}, \\
\lim _{\lambda \rightarrow 0} h_{i i}(\lambda)=\sum_{j=1}^{n-2} b_{i j}^{2}\left(1+d_{j} / n \lambda\right)^{-1}=0
\end{gathered}
$$




$$
\lim _{\lambda \rightarrow 0} h_{i j}(\lambda)=-\sum_{k=1}^{n-2} b_{i k} b_{j k}\left(1+d_{k} / n \lambda\right)^{-1}=0 .
$$

Intuitivamente, é possível notar em (2.36) que à medida que aumentamos o tamanho da amostra ou diminuímos o parâmetro de suavização, estamos assintoticamente aproximando $h_{i i}(\lambda)$ de $h_{i i}^{*}$, ou seja, estamos aproximando $\mathbf{H}(\lambda)$ para a matriz idempotente $\mathbf{H}^{*}$ com todas as propriedades do modelo linear usual, sendo assim possível a utilização dos elementos da matriz $\mathbf{H}(\lambda)$ para a análise de diagnóstico dos modelos com ajuste via splines cúbicos.

\subsubsection{Pontos de alavanca}

Um dos objetivos da análise de diagnóstico é a detecção de observações influentes na estimação dos parâmetros de um modelo. Hoaglin e Welsch [1978] realizaram o estudo da diagonal principal da matriz hat nos modelos lineares normais motivando a definição dos pontos de alavanca, cujo nome faz referência ao peso desproporcional que esses pontos exercem na estimativa dos parâmetros do modelo. Geralmente os pontos de alavanca possuem perfil diferente das demais observações no que diz respeito aos valores das variáveis explicativas e podem exercer forte influência na estimação dos parâmetros da regressão.

Com os resultados obtidos por Eubank [1984] descritos na seção anterior, também podemos detectar pontos de alavanca no caso do MANS. Seja $\widehat{y}_{i}$ o valor ajustado de $y_{i}$. Por (2.33), podemos escrever

$$
\widehat{y}_{i}=h_{i i}(\lambda) y_{i}+\sum_{i \neq j} h_{j i}(\lambda) y_{j}
$$

em que altos valores de $h_{i i}(\lambda)$ aumentam a influência de $y_{i}$ em sua própria predição. Supondo que todos os pontos $h_{i i}(\lambda)$ exerçam a mesma influência no ajuste, espera-se que os valores dos elementos da diagonal principal $h_{i i}(\lambda)$ estejam próximos de $\frac{\operatorname{tr}\{\mathbf{H}(\lambda)\}}{n}$, ou seja, uma forma de detectar pontos de alavanca é examinar, por exemplo, valores de $h_{i i}(\lambda) \geq \frac{2 \operatorname{tr}\{\mathbf{H}(\lambda)\}}{n}$.

\subsubsection{Resíduos}

Como definido anteriormente, os resíduos ordinários procuram medir a discrepância entre os valor observado e o valor ajustado do $i$-ésimo. No MANS, esses resíduos possuem expressão dada por

$$
\mathbf{r}=(\mathbf{I}-\mathbf{H}(\lambda)) \mathbf{y}
$$

Cada resíduo $r_{i}$ possui distribuição Normal com média zero e, como prova Eubank [1984], $\operatorname{Var}\left(r_{i}\right)=\sigma^{2}\left(1-h_{i i}(\lambda)\right)$, que é equivalente à variância dos resíduos do modelo linear usual mesmo sem a propriedade de idempotência da matriz $\mathbf{H}(\lambda)$. Como $(\mathbf{I}-\mathbf{H}(\lambda))$ não é uma matriz diagonal, temos que os resíduos são correlacionados e possuem covariâncias dadas por $\operatorname{Cov}\left(r_{i} \cdot r_{j}\right)=-\sigma^{2}\left(1-h_{i j}(\lambda)\right)$ para $i \neq j$. 
Assim, como no modelo linear usual, é aconselhável a padronização dos resíduos pelo respectivo desvio padrão para melhor comparação entre eles. Seja $\mathbf{r}^{*}$ o vetor de resíduos padronizados com elementos $r_{i}^{*}$, dados por

$$
r_{i}^{*}=\frac{r_{i}}{\sigma\left(1-h_{i i}(\lambda)\right)^{1 / 2}}
$$

sendo $\sigma$ a raiz quadrada do estimador de $\sigma^{2}$. Na prática, substituimos $\sigma^{2}$ por $s^{2}$ definido em (2.24).

Para maiores detalhes sobre o estudo da matriz $\mathbf{H}(\lambda)$ em MANS e derivações que permitem o uso das técnicas conhecidas de diagnóstico podem ser obtidos em Eubank [1984], que ainda sugere uma vasta bibliografia adicional. Para mais detalhes sobre técnicas de diagnóstico em modelos normais usuais e modelos lineares generalizados, recomenda-se Paula [2010].

\subsubsection{Influência}

A medida de influência mais conhecida para detectar pontos influentes no modelo normal linear é a distância de Cook [1977]. Nela, avaliamos a diferença entre a estimativa dos parâmetros com os dados completos e quando a $i$-ésima observação é desconsiderada. Eubank [1988] definiu uma distância de Cook análoga para o MANS, que tem expressão dada por

$$
D_{i}=\frac{\left(\widehat{\boldsymbol{\mu}}-\widehat{\boldsymbol{\mu}}_{(i)}\right)^{T} \mathbf{H}(\lambda)^{-1}\left(\boldsymbol{\mu}-\widehat{\boldsymbol{\mu}}_{(i)}\right)}{s^{2} \operatorname{tr}\{\mathbf{H}(\lambda)\}},
$$

em que $\widehat{\boldsymbol{\mu}}=\left(\widehat{\mu}_{1}, \ldots, \widehat{\mu}_{n}\right)^{T}$ é o vetor de estimativa das médias do modelo completo, com $\widehat{\boldsymbol{\mu}}=\mathbf{N} \widehat{\mathbf{f}}$, e $\widehat{\boldsymbol{\mu}}_{(i)}$ é o vetor de estimativas das médias do ajuste do spline desconsiderando a $i$-ésima observação.

A expressão (2.45) pode ser escrita de forma mais simplificada, levando em consideração as informações dos resíduos e os pontos de alavanca da $i$-ésima observação

$$
D_{i}=\frac{r_{i}^{2} h_{i i}(\lambda)}{s^{2} \operatorname{tr}\{\mathbf{H}(\lambda)\}\left(1-h_{i i}(\lambda)\right)^{2}} .
$$

Para um conjunto $K=\left\{i_{1}, \ldots, i_{K}\right\}$ de observações deletadas, temos uma expressão equivalente a (2.46) desenvolvida por Kim [1996] dada por

$$
D_{K}=\frac{\mathbf{r}_{K}^{T}\left(\mathbf{I}-\mathbf{H}(\lambda)_{(K)}\right)^{-1} \mathbf{H}_{(K)}\left(\mathbf{I}-\mathbf{H}_{(K)}\right)^{-1} \mathbf{r}_{K}}{s^{2} \operatorname{tr}\{\mathbf{H}(\lambda)\}} .
$$

Assim, como no modelo linear usual, valores altos de $D_{i}$ indicam alta influência da $i$-ésima observação no ajuste, ou seja, a exclusão desse ponto pode resultar em importantes alterações nas interpretações dos modelos.

\subsection{Estimação do parâmetro de suavização}

Nas seções anteriores, todas as derivações e inferências para a função $f(\cdot)$ consideravam o parâmetro de suavização $\lambda$ fixo, porém esse parâmetro também precisa ser estimado. Segundo 
Green e Silverman [1994], existem dois caminhos para a escolha do parâmetro de suavização. A primeira considera a livre escolha desse parâmetro, muitas vezes obtido após o teste de vários valores para o mesmo conjunto de dados e analisando as curvas obtidas. A segunda consiste em utilizarmos algum método automático em que o parâmetro de suavização é obtido através dos dados. Nessa dissertação, não discutiremos a livre escolha do parâmetro de suavização, e um exemplo ilustrativo desse procedimento pode ser encontrado em Lima [2001].

Atualmente existem diversos métodos para a escolha do parâmetro de suavização, e discutiremos dois deles: o método de validação cruzada (VC) e o método de validação cruzada generalizada (VCG). Mais detalhes referentes aos demais métodos de estimação do parâmetro de suavização podem ser encontrados, por exemplo, em Buja et al. [1989], Hastie e Tibshirani [1990], Rigby e Stasinopoulos [2005], entre outros.

\subsubsection{Validação cruzada}

O método de validação cruzada tem como objetivo estimar o erro de predição de um modelo ajustado aos dados. Esse método utiliza parte dos dados para a construção do modelo e o restante para a sua validação, ou seja, parte dos dados são considerados na avaliação da adequabilidade do ajuste. Segundo Green e Silverman [1994], quando a estimativa da função $f(\cdot)$ é um SCN, a expressão de cálculo da validação cruzada é dada por

$$
\mathrm{VC}(\lambda)=n^{-1} \sum_{i=1}^{n}\left(\frac{y_{i}-\widehat{y}_{i}}{1-h_{i i}(\lambda)}\right)^{2},
$$

em que $\widehat{y}=\left(\widehat{y}_{1}, \ldots, \widehat{y}_{n}\right)$ são as estimativas de $y$ dado um $\lambda$ fixo e $h_{i i}(\lambda)$ é o $i$-ésimo elemento da matriz $\mathbf{H}(\lambda)$ definida em (2.19). A ideia básica da validação cruzada é escolher $\lambda$ que minimize $\operatorname{VC}(\lambda)$. No entanto, como não temos a garantia que a função $\operatorname{VC}(\lambda)$ possui um único mínimo, é indicado realizar a inspeção dessa função. Além disso, qualquer método de minimização utilizado envolverá o cálculo de $\operatorname{VC}(\lambda)$ para um número de valores de $\lambda$, ou seja, é importante que se utilize um método eficiente para esse cálculo.

\subsubsection{Validação cruzada generalizada}

A validação cruzada generalizada (VCG) é uma forma modificada da validação cruzada bastante utilizada para a escolha do parâmetro de suavização. Diferente da equação (2.48), a ideia básica da validação cruzada generalizada é substituir o respectivo valor $h_{i i}(\lambda)$ de $\mathbf{H}(\lambda)$ por sua média $n^{-1} \operatorname{tr}\{\mathbf{H}(\lambda)\}$. Esse método o torna mais eficiente computacionalmente, já que é possível encontrar o traço de $\mathbf{H}(\lambda)$ sem necessariamente encontrar todos os valores de cada elemento $h_{i i}$. Dessa forma, podemos escrever a expressão da VCG como

$$
\operatorname{VCG}(\lambda)=n^{-1} \sum_{i=1}^{n}\left(\frac{y_{i}-\widehat{y}_{i}}{1-n^{-1} \operatorname{tr}\{\mathbf{H}(\lambda)\}}\right)^{2}
$$

Da mesma forma que na validação cruzada, a escolha do parâmetro de suavização para a vali- 
dação cruzada generalizada é feita pela minimização de $\operatorname{VCG}(\lambda)$.

Green e Silverman [1994] apresentam também uma expressão modificada do escore VCG $(\lambda)$, que leva em consideração os pontos de alavanca da matriz $\mathbf{H}(\lambda)$. Diferente da validação cruzada generalizada, em que todos os resíduos possuem o mesmo peso, a validação cruzada generalizada modificada pondera o resíduo adicionando ao numerador o termo $\left(1-h_{i i}\right)^{2}$, de forma que os resíduos gerados por pontos de alavanca tenham influência menor no cálculo do $\operatorname{VCG}(\lambda)$, ou seja,

$$
\operatorname{VCG}_{M}(\lambda)=n^{-1} \sum_{i=1}^{n}\left\{\left(\frac{1-h_{i i}(\lambda)}{1-n^{-1} \operatorname{tr}\{\mathbf{H}(\lambda)\}}\right)^{2}\left(y_{i}-\widehat{y}_{i}(\lambda)\right)^{2}\right\} .
$$

\subsection{Seleção de modelos}

Em analogia ao caso paramétrico, Rigby e Stasinopoulos [2005] sugerem um critério de seleção de modelos baseado no critério de informação de Akaike [1974], já que os parâmetros do modelo estudado neste capítulo são estimados via máxima verossimilhança. Para o caso do MANS, esses critérios foram adaptados utilizando o desvio global penalizado baseado no logaritmo da função de verossimilhança penalizada, criando os critérios de informação de Akaike penalizado ( $\left.\mathrm{AIC}_{p}\right)$ e o critério de informação de Schwarz penalizado $\left(\mathrm{SIC}_{p}\right)$. Da mesma forma que no modelo linear usual, a ideia desses critérios é priorizar modelos com menor número de parâmetros com o acréscimo de funções. Os critérios $\mathrm{AIC}_{p}$ e $\mathrm{SIC}_{p}$ são dados por

$$
\begin{aligned}
& \mathrm{AIC}_{p}=\mathrm{DG}_{p}+2 \operatorname{tr}\{\mathbf{H}(\lambda)\} \quad \mathrm{e} \\
& \mathrm{SIC}_{p}=\mathrm{DG}_{p}+\operatorname{tr}\{\mathbf{H}(\lambda)\} \log n,
\end{aligned}
$$

em que $\mathrm{DG}_{p}=-2 \ell_{p}(\boldsymbol{\theta}, \lambda)$, com $\ell_{p}(\boldsymbol{\theta}, \lambda)$ sendo o logaritmo da função de verossimilhança penalizada para o estimador de $\boldsymbol{\theta}, \operatorname{tr}\{\mathbf{H}(\lambda)\}$ denota os graus de liberdade do ajuste e $n$ é o total de observações na amostra.

Nos dois critérios sugeridos, maximizar a função de máxima verossimilhança penalizada é equivalente a minimizar o critério de seleção, ou seja, dentre os modelos considerados, devemos escolher o que possui o menor valor para esses critérios.

\subsection{Suavizadores ponderados}

Até agora, toda a teoria do MANS apresentada supõe a mesma variância para todas as observações. Porém, quando a suposição de igualdade de variâncias não é verificada, o ideal é adotarmos um método de suavização ponderado em que a $i$-ésima observação é associada à um peso $w_{i}$. Esses pesos são determinados de acordo com cada situação. Por exemplo, assumindo $\sigma_{i}^{2}$ a variância (conhecida) associada à $i$-ésima observação, o peso atribuído à $i$-ésima observação será $w_{i}=1 / \sigma_{i}^{2}$, com $\sigma_{i}^{2}$ conhecido. Outra situação é quando temos uma quantidade razoável de observações empatadas, em que o $w_{i}$ é determinado pela quantidade de repetições de $t_{i}^{0}, i=1, \ldots, r$. Nesta seção, adaptaremos 
os resultados das seções anteriores para o caso ponderado. Seja o modelo dado em (2.1). A soma dos quadrados dos erros ponderada sob a curva $f(\cdot)$ é dada por

$$
\sum_{i=1}^{n} w_{i}\left[y_{i}-f\left(t_{i}\right)\right]^{2}=(\mathbf{y}-\mathbf{N f})^{T} \mathbf{W}(\mathbf{y}-\mathbf{N f}),
$$

em que $\mathbf{W}$ é a matriz diagonal $(n \times n)$ de pesos $w_{i}$ conhecidos. Nesse caso, a minimização da soma dos quadrados dos erros ponderada penalizada (SQEPP) consiste em minimizar

$$
(\mathbf{y}-\mathbf{N f})^{T} \mathbf{W}(\mathbf{y}-\mathbf{N f})+\lambda \mathbf{f}^{T} \mathbf{K} \mathbf{f}
$$

ou maximizar

$$
\ell_{p}(\boldsymbol{\theta}, \lambda)=-\frac{n}{2} \log 2 \pi \sigma^{2}-\frac{1}{2 \sigma^{2}}(\mathbf{y}-\mathbf{N f})^{T} \mathbf{W}(\mathbf{y}-\mathbf{N f})-\frac{1}{2} \lambda \mathbf{f}^{T} \mathbf{K f},
$$

para $\lambda^{*}=\lambda / \sigma^{2}$. O estimador de máxima verossimilhança penalizado ponderado de $\mathbf{f}$ é

$$
\widehat{\mathbf{f}}=\left(\mathbf{N}^{T} \widehat{\mathbf{W}} \mathbf{N}+\lambda \mathbf{K}\right)^{-1} \mathbf{N}^{T} \widehat{\mathbf{W}} \mathbf{y}=\mathbf{S}(\lambda) \mathbf{y}
$$

e $\mathbf{H}(\lambda)$ é a matriz hat $(n \times n)$ simétrica dada por $\mathbf{H}(\lambda)=\mathbf{N S}=\mathbf{N}\left(\mathbf{N}^{T} \mathbf{W N}+\lambda \mathbf{K}\right)^{-1} \mathbf{N}^{T} \mathbf{W}$.

A estimação do parâmetro de suavização via validação cruzada generalizada também se modifica no caso ponderado. Análogo à (2.49), a escolha do parâmetro de suavização consiste em minimizar

$$
\operatorname{VCG}(\lambda)=n^{-1} \sum_{i=1}^{n} w_{i}\left(\frac{y_{i}-\widehat{y}_{i}(\lambda)}{1-n^{-1} \operatorname{tr}\{\mathbf{H}(\lambda)\}}\right)^{2}
$$

em que $\widehat{y}(\lambda)=\left(\widehat{y}_{1}(\lambda), \ldots, \widehat{y}_{n}(\lambda)\right)$ são as estimativas de $y$ via máxima verossimilhança penalizada ponderada dado um $\lambda$ fixo.

\subsection{Observações empatadas}

No início deste capítulo assumimos que os valores da variável explicativa eram distintos, porém é relativamente comum essa variável apresentar mais de uma observação $t_{i}$. Como definido em (2.2), seja $\mathbf{f}=\left(f\left(t_{1}\right)^{0}, \ldots, f\left(t_{r}^{0}\right)\right)^{T}$ o vetor de funções $(r \times 1)$ do efeito não paramétrico, em que cada elemento $t_{j}^{0}$ representa um valor ordenado e distinto da variável explicativa, $j=1, \ldots, r$. Nesse caso, podemos criar um novo conjunto de dados com $r \leq n$ observações, com variável resposta associada $\bar{y}_{j}$ à essa $j$-ésima observação dada pela média dos valores de $\mathbf{y}$ associados à esse grupo, ou seja,

$$
\bar{y}_{j}=m_{j}^{-1} \sum_{i=1}^{m_{j}} y_{i j}
$$


em que $m_{j}$ é a quantidade de réplicas da $j$-ésima observação. Nesse caso, também teremos que adotar um método de suavização ponderado para o novo conjunto de dados, em que a $j$-ésima observação é associada ao peso $w_{j}=m_{j}$.

\subsection{Considerações}

Este capítulo teve como objetivo principal revisitar toda a teoria referente aos modelos aditivos normais simples na literatura e entender principalmente os conceitos de splines e critérios de penalização, função de máxima verossimilhança penalizada e logaritmo da função de verossimilhança penalizada, estimação dos parâmetros de posição e dispersão, definição e estimação do parâmetro de suavização, e as possíveis medidas de diagnóstico para esse tipo de modelo. Como um dos objetivos deste trabalho é estudar métodos de diagnóstico em modelos semiparamétricos normais com uma função não paramétrica com estimação via splines cúbicos, todos os conceitos referentes ao MANS devem estar claros para melhor entendimento dos próximos capítulos. 


\section{Capítulo 3}

\section{Modelos semiparamétricos normais}

\subsection{Introdução}

Embora o modelo estudado no capítulo anterior seja suficiente para resolver uma variedade de problemas, na prática existem situações em que a resposta observada é simultaneamente dependente de diversas variáveis explicativas. A forma mais conhecida de se trabalhar com esse tipo de problema são os modelos lineares multidimensionais expressos por

$$
y_{i}=\mathbf{x}_{i}^{T} \boldsymbol{\beta}+\epsilon_{i},
$$

em que $y_{i}$ é a variável resposta referente à $i$-ésima observação, $\mathbf{x}_{i}$ é o vetor de valores das variáveis explicativas associadas, $\boldsymbol{\beta}$ é o vetor de parâmetros a ser estimado e $\epsilon_{i}$ é o $i$-ésimo erro aleatório não observável.

No entanto, quando não existe uma relação linear entre as variáveis explicativas e a variável resposta, o modelo (3.1) pode não ser uma boa solução. Nesses casos, os modelos não paramétricos podem ser uma alternativa, já que a suposição de linearidade necessária em (3.1) é relaxada, ou seja, não é necessária uma forma rígida de dependência entre essas variáveis. Considerando toda a teoria vista no capítulo anterior, uma analogia natural seria considerarmos o modelo

$$
y_{i}=f\left(\mathbf{x}_{i}\right)+\epsilon_{i}
$$

ou seja $f(\cdot)$ é, assim como vimos anteriormente, uma função suavizadora arbitrária dos efeitos não paramétricos. No entanto, os modelos de regressão não paramétricos multidimensionais apresentam alguns problemas conceituais e não são muito eficientes computacionalmente, além de serem de difícil interpretação.

Há também os modelos aditivos normais de expressão dada por

$$
y_{i}=\alpha+f_{1}\left(\mathbf{x}_{1 i}\right)+\ldots+f_{q}\left(\mathbf{x}_{q i}\right)+\epsilon_{i}
$$

em que $f_{1}(\cdot), \ldots, f_{q}(\cdot)$ são $q$ funções arbitrárias. Esses modelos são discutidos por Hastie e Tibshirani [1990], porém não serão tratados nesse trabalho. 
Já em Ibacache-Pulgar et al. [2013] são apresentados alguns procedimentos de diagnósticos para modelos aditivos simétricos, incluindo diagnósticos de influência local.

Neste capítulo trataremos apenas uma variável explicativa de forma não paramétrica, enquanto que o restante pode ser considerado analogamente como no modelo linear multidimensional usual. Esse tipo de modelo, conhecido como modelo semiparamétrico ou modelo linear parcial (MLP), tem aplicabilidade em diversas áreas e é bastante conhecido no contexto de dados longitudinais, já que estudos mostram que é comum observar um comportamento não linear da variável resposta em relação ao tempo.

Dentre os estudos referentes aos modelos lineares parciais, podemos destacar Heckman [1986], que mostra que os estimadores dos coeficientes paramétricos e não paramétrico são estimadores de Bayes quando assumimos uma distribuição a priori apropriada. Green e Silverman [1994] definem, e Green [1987] também estudam o comportamento assintótico dos estimadores de máxima verossimilhança penalizada. No contexto de métodos de diagnóstico, podemos destacar Kim et al. [2002], que apresentam medidas de influência baseadas nos resíduos, pontos de alavanca e distância de Cook dos componentes paramétricos e não paramétricos do modelo.

O objetivo deste capítulo é estudar detalhadamente os modelos lineares parciais, a estimação dos parâmetros referentes aos componentes paramétricos e não paramétricos e, principalmente, apresentar as técnicas de diagnóstico disponíveis para esse tipo de modelo.

\subsection{Especificação do modelo}

Como definido anteriormente, os MLPs são uma extensão do modelo linear usual e têm como principal característica a inclusão de um componente não paramétrico associado a alguma variável explicativa que não possui relação linear com a variável resposta.

Seja $y_{i}(i=1, \ldots, n)$ o valor da variável resposta contínua associado à $i$-ésima observação de uma amostra, e que para cada uma dessas observações há $(p-1)$ valores de variáveis explicativas e uma variável explicativa cujos valores $\left(t_{1}, \ldots t_{n}\right)$ são avaliados de forma não paramétrica. O MLP assume a forma

$$
y_{i}=\beta_{0}+\sum_{k=1}^{p-1} x_{i k} \beta_{k}+f\left(t_{i}\right)+\epsilon_{i}
$$

em que $\beta_{0}$ é o intercepto, $x_{i k}$ é o valor da $k$-ésima variável explicativa de comportamento paramétrico associada à $i$-ésima observação, $\beta_{k}$ é o $k$-ésimo parâmetro desconhecido, $f(\cdot)$ é uma função suavizadora duas vezes diferenciável que depende dos valores da variável explicativa de comportamento não paramétrico, e $\epsilon_{i}$ é o erro aleatório associado à $i$-ésima observação. Matricialmente esse modelo pode ser escrito como

$$
\mathbf{y}=\mathbf{X} \boldsymbol{\beta}+\mathbf{N f}+\epsilon
$$

sendo $\mathbf{y}=\left(y_{1}, \ldots, y_{n}\right)^{T}$ o vetor $(n \times 1)$ de respostas observadas independentes, $\mathbf{X}$ a matriz de 
planejamento $(n \times p)$ de linhas $\mathbf{x}_{i}^{T}, \boldsymbol{\beta}$ o vetor de parâmetros desconhecidos $(p \times 1)$ do componente paramétrico, $\mathbf{f}=\left(f\left(t_{1}^{0}\right), \ldots, f\left(t_{r}^{0}\right)\right)^{T}$ o vetor de funções $(r \times 1)$ do componente não paramétrico, com $t_{j}^{0}$ sendo o $j$-ésimo valor ordenado e distinto da variável explicativa $t, \mathbf{N}$ é a matriz de incidência $(n \times r)$ com elementos dados pela função indicadora $I\left(t_{i}=t_{k}^{0}\right)$, e $\boldsymbol{\epsilon}=\left(\epsilon_{1}, \ldots, \epsilon_{n}\right)^{T}$ o vetor de erros aleatórios $(n \times 1)$. Logo, temos as distribuições de $\mathbf{y}$ e $\boldsymbol{\epsilon}$ dadas por

$$
\left\{\begin{array}{l}
\mathbf{y} \sim \mathcal{N}_{n}\left(\mathbf{X} \boldsymbol{\beta}+\mathbf{N f}, \sigma^{2} \mathbf{I}\right) \quad \mathrm{e} \\
\boldsymbol{\epsilon} \sim \mathcal{N}_{n}\left(\mathbf{0}, \sigma^{2} \mathbf{I}\right) .
\end{array}\right.
$$

Diferentemente do capítulo anterior, neste capítulo consideraremos um modelo mais geral, em que os erros são ponderados. Uma justificativa para essa escolha é que, ao contrário do modelo (2.1), esse modelo possui $p$ variáveis explicativas e a suposição de igualdade de variâncias nem sempre é satisfeita. Neste trabalho consideraremos erros homocedásticos, porém o fator de ponderação usual para casos de heteroscedasticidade é dado pelo inverso da variância da $i$-ésima observação $\sigma_{i}^{2}$, ou seja, $w_{i}=1 / \sigma_{i}^{2}, \operatorname{com} \sigma_{i}^{2}$ conhecidos e $i=1, \ldots, n$.

\subsection{Critério de penalização}

Da mesma maneira que no MANS, o acréscimo de uma penalização na soma de quadrados dos erros é uma alternativa para avaliação da qualidade do ajuste do modelo, já que o ajuste tende a ser melhor quando a função $f(\cdot)$ interpolar todas as observações da amostra. Dessa forma, vamos considerar o método de suavização spline cúbico para a estimação do componente não paramétrico e a soma dos quadrados dos erros penalizada para a avaliação da qualidade do ajuste. Na Seção 3.3.1 definimos a função de penalização utilizada no contexto dos MLPs, e nas Seções 3.3.2 e 3.3.3 definimos, respectivamente, o logaritmo da função de verossimilhança penalizada, o vetor escore e a matriz de informação de Fisher.

\subsubsection{Função de penalização}

A soma dos quadrados dos erros penalizada para a avaliação da qualidade do ajuste tem expressão dada por

$$
\sum_{i=1}^{n} w_{i}\left[y_{i}-\mathbf{x}_{i}^{T} \boldsymbol{\beta}-f\left(t_{i}\right)\right]^{2}+\lambda^{*} \int_{a}^{b}\left[f^{\prime \prime}(t)\right]^{2} d t, \quad \lambda>0
$$

em que $\lambda$ é o parâmetro de suavização e, assim como no MANS, a priorização do ajuste ótimo ocorre quando $\lambda \rightarrow 0$, enquanto que a suavidade da curva é priorizada quando $\lambda \rightarrow \infty$. A solução de (3.6) é única e corresponde a um spline cúbico natural. Sua forma matricial é dada por

$$
(\mathbf{y}-\mathbf{X} \boldsymbol{\beta}-\mathbf{N f})^{T} \mathbf{W}(\mathbf{y}-\mathbf{X} \boldsymbol{\beta}-\mathbf{N f})-\lambda^{*} \mathbf{f}^{T} \mathbf{K f}
$$

em que $\mathbf{W}$ é a matriz de ponderação $(n \times n)$ e $\mathbf{K}$ é a matriz $(r \times r)$ positiva definida que depende apenas dos nós e que tem a mesma estrutura da matriz $\mathbf{K}$ dos MANSs. 


\subsubsection{Logaritmo da função de verossimilhança penalizada}

Seja $\boldsymbol{\theta}=\left(\boldsymbol{\beta}^{T}, \mathbf{f}^{T}, \sigma^{2}\right)^{T}$ o vetor de parâmetros a ser estimado no modelo (3.5), com espaços paramétricos $\Theta_{\mathbf{f}} \in \mathbb{R}, \Theta_{\beta} \in \mathbb{R}$ e $\Theta_{\sigma^{2}} \in \mathbb{R}^{+}$. A função de verossimilhança no MLP é o produto das funções densidade de probabilidade da distribuição normal com parâmetros $\mu_{i}=\mathbf{x}_{i}^{T} \boldsymbol{\beta}+f\left(t_{i}\right)$ e $\sigma^{2}$, dada por

$$
L(\boldsymbol{\theta})=\prod_{i=1}^{n} \frac{1}{\sigma \sqrt{2 \pi}} \exp \left\{-\frac{1}{2 \sigma^{2}}\left(y_{i}-\mathbf{x}_{i}^{T} \boldsymbol{\beta}-f\left(t_{i}\right)\right)^{2}\right\}
$$

Como dito na seção anterior, o acréscimo de uma função de penalização é necessário para obter uma boa estimação da curva $f(\cdot)$. Seja $\ell_{p}(\boldsymbol{\theta}, \lambda)$ o logaritmo da função de verossimilhança penalizada

$$
\ell_{p}(\boldsymbol{\theta}, \lambda)=-\frac{n}{2} \log 2 \pi \sigma^{2}-\frac{1}{2} \sum_{i=1}^{n} w_{i} \frac{\left(y_{i}-\mathbf{x}_{i}^{T} \boldsymbol{\beta}-f\left(t_{i}\right)\right)^{2}}{\sigma^{2}}+\frac{\lambda}{2} \int_{a}^{b} f^{\prime \prime}\left(t_{i}\right)^{2},
$$

que tem forma matricial dada por

$$
\ell_{p}(\boldsymbol{\theta}, \lambda)=-\frac{n}{2} \log 2 \pi \sigma^{2}-\frac{1}{2 \sigma^{2}}(\mathbf{y}-\mathbf{X} \boldsymbol{\beta}-\mathbf{N f})^{T} \mathbf{W}(\mathbf{y}-\mathbf{X} \boldsymbol{\beta}-\mathbf{N f})-\frac{1}{2} \lambda \mathbf{f}^{T} \mathbf{K f} .
$$

Os estimadores de máxima verossimilhança de $\mathbf{f}, \boldsymbol{\beta}$ e $\sigma^{2}$ são os vetores $\widehat{\mathbf{f}}, \widehat{\boldsymbol{\beta}}$ e $\widehat{\sigma}^{2}$ que maximizam o logaritmo da função de verossimilhança penalizada (3.10) e, da mesma forma que no MANS, a maximização de $\ell_{p}(\boldsymbol{\theta}, \lambda)$ para $\lambda=\lambda^{*} / \sigma^{2}$ é equivalente à minimização da soma de quadrados dos erros penalizada dada em (3.6).

\subsubsection{Função escore e informação de Fisher penalizada}

Por ora, vamos considerar $\lambda$ fixo e seja $\mathbf{U}_{p}(\boldsymbol{\theta})$ o vetor de funções escore penalizadas $\left(p^{\prime} \times 1\right)$, com $p^{\prime}=p+r+1$, dado por

$$
\mathbf{U}_{p}(\boldsymbol{\theta})=\left(\begin{array}{c}
\mathbf{U}_{p}^{\boldsymbol{\beta}}(\boldsymbol{\theta}) \\
\mathbf{U}_{p}^{\mathbf{f}}(\boldsymbol{\theta}) \\
\mathrm{U}_{p}^{\sigma^{2}}(\boldsymbol{\theta})
\end{array}\right)
$$

em que $\mathbf{U}_{p}^{\boldsymbol{\beta}}(\boldsymbol{\theta})$ é o vetor $(p \times 1)$ de funções escore referente à parte paramétrica, $\mathbf{U}_{p}^{\mathbf{f}}(\boldsymbol{\theta})$ é o vetor $(r \times 1)$ de funções escore referente à parte não paramétrica, e $\mathrm{U}_{p}^{\sigma^{2}}(\boldsymbol{\theta})$ é a função escore referente ao parâmetro de dispersão. Após algumas manipulações algébricas, verificamos que

$$
\begin{aligned}
\mathbf{U}_{p}^{\boldsymbol{\beta}}(\boldsymbol{\theta}) & =\sigma^{-2} \mathbf{X}^{T} \mathbf{W}(\mathbf{y}-\mathbf{X} \boldsymbol{\beta}-\mathbf{N f}) \\
\mathbf{U}_{p}^{\mathbf{f}}(\boldsymbol{\theta}) & =\sigma^{-2} \mathbf{N}^{T} \mathbf{W}(\mathbf{y}-\mathbf{X} \boldsymbol{\beta}-\mathbf{N f})-\lambda \mathbf{K f} \quad \mathrm{e} \\
\mathrm{U}_{p}^{\sigma^{2}}(\boldsymbol{\theta}) & =\frac{1}{2 \sigma^{2}}\left[\frac{(\mathbf{y}-\mathbf{X} \boldsymbol{\beta}-\mathbf{N f})^{T} \mathbf{W}(\mathbf{y}-\mathbf{X} \boldsymbol{\beta}-\mathbf{N f})}{\sigma^{2}}-n\right]
\end{aligned}
$$


Seja $\mathcal{I}_{p}(\boldsymbol{\theta})$ a matriz de informação de Fisher $\left(p^{\prime} \times p^{\prime}\right)$ definida como

$$
\mathcal{I}_{p}(\boldsymbol{\theta})=\left(\begin{array}{cc}
\mathcal{I}_{p}^{\boldsymbol{\beta f}}(\boldsymbol{\theta}) & \mathbf{0} \\
0 & \mathcal{I}_{p}^{\sigma^{2} \sigma^{2}(\boldsymbol{\theta})}
\end{array}\right)
$$

em que $\mathcal{I}_{p}^{\boldsymbol{\beta f}}(\boldsymbol{\theta})$ é dada por

$$
\mathcal{I}_{p}^{\boldsymbol{\beta f}}(\boldsymbol{\theta})=\left(\begin{array}{cc}
\mathbf{X}^{T} \mathbf{W X} & \mathbf{X}^{T} \mathbf{W} \mathbf{N} \\
\mathbf{N}^{T} \mathbf{W X} & \mathbf{N}^{T} \mathbf{W} \mathbf{N}+\lambda \sigma^{2} \mathbf{K}
\end{array}\right),
$$

e a matriz de informação de Fisher com relação ao parâmetro de dispersão fica dada por

$$
\mathcal{I}_{p}^{\sigma^{2} \sigma^{2}}(\boldsymbol{\theta})=\frac{n}{2 \sigma^{4}}
$$

Para detalhes referentes aos cálculos das funções escores e informações de Fisher para o MLP, consultar o Apêndice C.

\subsection{Estimação dos parâmetros}

A estimação dos parâmetros de posição dos MLPs normais pode ser realizada de duas maneiras: via algoritmo backfitting ou pelo método direto através da maximização da função de verossimilhança penalizada.

\subsubsection{Estimação dos parâmetros de posição}

Supondo que o logaritmo da função de verossimilhança penalizada $\ell_{p}(\boldsymbol{\theta}, \lambda)$ definido em (3.10) é uma função duas vezes diferenciável e que satisfaz certas condições de regularidade, o vetor paramétrico $\boldsymbol{\beta}$ e o vetor não paramétrico f que maximizam $\ell_{p}(\boldsymbol{\theta}, \lambda)$ são EMVPs $\widehat{\boldsymbol{\beta}}$ e $\widehat{\mathbf{f}}$ que satisfazem a desigualdade

$$
\ell_{p}(\widehat{\boldsymbol{\theta}}, \lambda) \geq \sup _{\boldsymbol{\theta} \in \Theta} \ell_{p}(\boldsymbol{\theta}, \lambda)
$$

Considere $\lambda$ e $\sigma^{2}$ fixos. As soluções das equações de estimação que conduzem aos EMVPs de $\boldsymbol{\beta}$ e f são dadas por

$$
\begin{gathered}
\mathbf{U}_{p}^{\boldsymbol{\beta}}(\boldsymbol{\theta})=\sigma^{-2} \mathbf{X W}(\mathbf{y}-\mathbf{X} \boldsymbol{\beta}-\mathbf{N f})=\mathbf{0} \\
\mathbf{U}_{p}^{\mathbf{f}}(\boldsymbol{\theta})=\sigma^{-2} \mathbf{N W}(\mathbf{y}-\mathbf{X} \boldsymbol{\beta}-\mathbf{N f})-\lambda \mathbf{K f}=\mathbf{0},
\end{gathered}
$$

que possuem solução única [Green e Silverman, 1994]. Depois de algumas manipulações algébricas, chegamos em

$$
\boldsymbol{\beta}=\left(\mathbf{X}^{T} \mathbf{W} \mathbf{X}\right)^{-1} \mathbf{X}^{T} \mathbf{W}(\mathbf{y}-\mathbf{N f}) \quad \mathrm{e}
$$




$$
\mathbf{f}=\left(\mathbf{N}^{T} \mathbf{W N}+\lambda \mathbf{K}\right)^{-1} \mathbf{N}^{T} \mathbf{W}(\mathbf{y}-\mathbf{X} \boldsymbol{\beta})
$$

As expressões (3.12) e (3.13) não possuem solução analítica explícita. Porém, intuitivamente podemos observar que, se o estimador $\widehat{\mathbf{f}}$ for conhecido e considerado constante em $(3.12),(\mathbf{y}-\mathbf{N f})$ é uma estimativa de $\boldsymbol{\beta}$ via máxima verossimilhança penalizada dessas diferenças. Da mesma forma, se $\widehat{\boldsymbol{\beta}}$ é conhecido, (3.13) nos diz à respeito do ajuste do spline cúbico via máxima verossimilhança penalizada das diferenças $(\mathbf{y}-\mathbf{X} \boldsymbol{\beta})$. Essa interpretação pode ser levada adiante, no sentido que podemos fazer repetidamente uma alternância entre (3.12) e (3.13) para estimar $\widehat{\boldsymbol{\beta}}$ e $\widehat{\mathbf{f}}$ via máxima verossimilhança penalizada até a convergência desejada. Esse algoritmo é conhecido como backfitting, e foi introduzido por Breiman e Friedman [1985]. Para maiores detalhes referentes ao algoritmo backfitting, consultar Green et al. [1985] ou Buja et al. [1989].

Resumidamente, podemos realizar a estimação da seguinte forma: estimamos a parte paramétrica do modelo via máxima verossimilhança usual e obtemos o valor inicial $\boldsymbol{\beta}^{(0)}$. O resíduo associado ao ajuste paramétrico é usado como resposta na estimação via máxima verossimilhança penalizada de $\mathbf{f}^{(0)}$. Assim, a iteração do algoritmo é realizada até a convergência. A Tabela 3.1 nos dá uma ideia de como o algoritmo funciona em sua $(u+1)$-ésima etapa:

Tabela 3.1: $(u+1)$-ésima etapa do algoritmo backfitting sob o MLP.

(i) Estimação de $\boldsymbol{\beta}^{(0)}$ via máxima verossimilhança e obtenção de $\mathbf{f}^{(0)}$;

(ii) para $u=0,1,2, \ldots$, calcular:

(ii') $\quad \mathbf{f}^{(u+1)}=\left(\mathbf{N}^{T} \mathbf{W}^{(u)} \mathbf{N}+\lambda \mathbf{K}\right)^{-1} \mathbf{N}^{T} \mathbf{W}^{(u)}\left(\mathbf{y}-\mathbf{X} \boldsymbol{\beta}^{(u)}\right) ;$

(ii”) $\quad \boldsymbol{\beta}^{(u+1)}=\left(\mathbf{X}^{T} \mathbf{W}^{(u)} \mathbf{X}\right)^{-1} \mathbf{X}^{T} \mathbf{W}^{(u)}\left(\mathbf{y}-\mathbf{N} \mathbf{f}^{(u)}\right)$

(iii) Repetir (ii) até a convergência.

Green e Silverman [1994] provam que, sob determinadas condições relacionadas às propriedades das matrizes $\mathbf{N}, \mathbf{W}$ e $\mathbf{K}$, o algoritmo backfitting sempre converge e pode terminar em pouco mais de 5 iterações. Além disso, eles também propõem um método de estimação sem iteração, que além de possuir uma forma fechada para o estimador de $\boldsymbol{\beta}$ e $\mathbf{f}$, é bastante útil no que diz respeito às análises de pontos influentes para os MLPs. Essa forma fechada é obtida substituindo (3.13) em (3.12), e após algumas manipulações algébricas obtém-se

$$
\widehat{\boldsymbol{\beta}}=\left(\mathbf{X}^{T} \mathbf{W}_{x} \mathbf{X}\right)^{-1} \mathbf{X}^{T} \mathbf{W}_{x} \mathbf{y}
$$

$\operatorname{com} \mathbf{W}_{x}=\mathbf{W}-\mathbf{W N}\left(\mathbf{N}^{T} \mathbf{W N}+\lambda \mathbf{K}\right)^{-1} \mathbf{N}^{T} \mathbf{W}$, e consequentemente temos

$$
\widehat{\mathbf{f}}=\left(\mathbf{N}^{T} \mathbf{W}_{f} \mathbf{N}+\lambda \mathbf{K}\right)^{-1} \mathbf{N}^{T} \mathbf{W}_{f} \mathbf{y}
$$


com $\mathbf{W}_{f}=\mathbf{W}-\mathbf{W X}\left(\mathbf{X}^{T} \mathbf{W X}\right)^{-1} \mathbf{X}^{T} \mathbf{W}$. Consequentemente, utilizando as expressões (3.14) e (3.15) e após várias manipulações algébricas, o vetor de valores ajustados $\widehat{\mathbf{y}}$ pode ser escrito na forma

$$
\widehat{\mathbf{y}}=\mathbf{X} \widehat{\boldsymbol{\beta}}+\mathbf{N} \widehat{\mathbf{f}}=\overline{\mathbf{H}}(\lambda) \mathbf{y}
$$

com $\overline{\mathbf{H}}(\lambda)$ definida como matriz hat do MLP, com expressão dada por

$$
\overline{\mathbf{H}}(\lambda)=\left(\begin{array}{cc}
\mathbf{X} & \mathbf{N}
\end{array}\right)\left(\begin{array}{cc}
\mathbf{X}^{T} \mathbf{W X} & \mathbf{X}^{T} \mathbf{W} \mathbf{N} \\
\mathbf{N}^{T} \mathbf{W X} & \mathbf{N}^{T} \mathbf{W} \mathbf{N}+\lambda \mathbf{K}
\end{array}\right)^{-1}\left(\begin{array}{c}
\mathbf{X}^{T} \\
\mathbf{N}^{T}
\end{array}\right) \mathbf{W}
$$

que, assim como no capítulo anterior, essa matriz será útil para a análise de diagnóstico, especialmente para a análise de pontos de alavanca, influentes e análise de resíduos.

\subsubsection{Graus de liberdade}

Assim como no capítulo anterior, existe a necessidade do cálculo dos graus de liberdade efetivos (GLE), que dá uma indicação do número efetivo de parâmetros considerados no ajuste do MLP. Dessa forma, define-se os GLE associados à regressão como

$$
\mathrm{GLE}=\operatorname{tr}\{\overline{\mathbf{H}}(\lambda)\}
$$

Como o cálculo do GLE depende do parâmetro de suavização $\lambda$ no componente não paramétrico, se supormos $\lambda \rightarrow 0$, estaremos priorizando o ajuste do modelo com a interpolação das $n$ observações, ou seja, GLE $\rightarrow n$. Porém, quando $\lambda \rightarrow \infty$, nosso modelo será equivalente ao modelo de regressão linear simples usual, ou seja, GLE $=p+1$. Nesse caso, temos que o domínio de GLE é o intervalo $[p+1 ; n]$.

\subsubsection{Estimação do parâmetro de dispersão}

Para a estimação do parâmetro $\sigma^{2}$, considere os EMVPs $\widehat{\boldsymbol{\beta}}$ e $\widehat{\mathbf{f}}$ obtidos na Seção 3.4.1. Igualando a função escore parcial $U_{p}^{\sigma^{2}}$ a zero , obtém-se o estimador

$$
\widehat{\sigma}^{2}=\frac{(\mathbf{y}-\mathbf{X} \widehat{\boldsymbol{\beta}}-\mathbf{N} \widehat{\mathbf{f}})^{T}(\mathbf{y}-\mathbf{X} \widehat{\boldsymbol{\beta}}-\mathbf{N} \widehat{\mathbf{f}})}{n} .
$$

No entanto, o estimador (3.19) é viesado e, nesse caso, o mais apropriado é utilizarmos o estimador amostral $s^{2}$, dado pela razão entre a soma dos quadrados dos resíduos e o GLE associado aos resíduos, ou seja,

$$
s^{2}=\frac{(\mathbf{y}-\mathbf{X} \widehat{\boldsymbol{\beta}}-\mathbf{N} \widehat{\mathbf{f}})^{T}(\mathbf{y}-\mathbf{X} \widehat{\boldsymbol{\beta}}-\mathbf{N} \widehat{\mathbf{f}})}{\operatorname{tr}\{\mathbf{I}-\overline{\mathbf{H}}(\lambda)\}} .
$$

Para detalhes referentes à $U_{p}^{\sigma^{2}}$, ver Apêndice C. 


\subsubsection{Estimação do parâmetro de suavização}

Na Seção 2.8 discutimos o uso da estimação via validação cruzada generalizada para a estimação do parâmetro de suavização nos MANS. No caso dos MLPs, usaremos a mesma técnica de estimação com as modificações apropriadas, de forma que a expressão a ser minimizada fique dada por

$$
\operatorname{VCG}(\lambda)=\sum_{i=1}^{n} w_{i}\left(\frac{y_{i}-\widehat{y}_{i}}{1-n^{-1} \operatorname{tr}\{\overline{\mathbf{H}}(\lambda)\}}\right)^{2}
$$

em que $\widehat{\mathbf{y}}=\left(\widehat{y}_{1}, \ldots, \widehat{y}_{n}\right)^{T}$ é o vetor das estimativas de $y_{i}$ e $n^{-1} \operatorname{tr}\{\overline{\mathbf{H}}(\lambda)\}$ é a média do traço da matriz hat obtida em (3.17). A escolha do parâmetro de suavização é realizada pela minimização de $\operatorname{VCG}(\lambda)$.

\subsection{Inferências para $\beta$ e f}

Antes de estudarmos a inferência no MLP, é importante lembrar que o MLP não possui toda a teoria assintótica conhecida das classes de modelos lineares usuais. Nesse tipo de modelo, várias premissas são relaxadas, como a normalidade, a parametrização linear, e segundo Green [1987], um estatístico matemático poderá ter sérios problemas para entender toda a teoria disponível para esse tipo de modelo. Ainda segundo Green, boa parte da teoria assintótica para o MLP é especulação.

Nas próximas seções, obteremos a distribuição dos estimadores $\widehat{\boldsymbol{\beta}}$ e $\widehat{\mathbf{f}}$ e seus respectivos erros padrão aproximados.

\subsubsection{Distribuição de $\widehat{\boldsymbol{\beta}}$ e $\widehat{\mathrm{f}}$}

Por definição, o estimador $\widehat{\boldsymbol{\theta}}$ é dito não viesado para $\boldsymbol{\theta}$ se $\mathrm{E}(\widehat{\boldsymbol{\theta}})=\boldsymbol{\theta}$. Considerando os estimadores obtidos em (3.14) e (3.15), temos que a esperança dos estimadores $\widehat{\mathbf{f}}$ e $\widehat{\boldsymbol{\beta}}$ são dados, respectivamente, por

$$
\begin{aligned}
\mathrm{E}(\widehat{\mathbf{f}}) & =\mathrm{E}\left[\left(\mathbf{N}^{T} \mathbf{W}_{f} \mathbf{N}+\lambda \mathbf{K}\right)^{-1} \mathbf{N}^{T} \mathbf{W}_{f} \mathbf{y}\right] \\
& =\left(\mathbf{N}^{T} \mathbf{W}_{f} \mathbf{N}+\lambda \mathbf{K}\right)^{-1} \mathbf{N}^{T} \mathbf{W}_{f}(\mathbf{X} \boldsymbol{\beta}+\mathbf{N f}),
\end{aligned}
$$

e

$$
\begin{aligned}
\mathrm{E}(\widehat{\boldsymbol{\beta}}) & =\mathrm{E}\left[\left(\mathbf{X}^{T} \mathbf{W}_{x} \mathbf{X}\right)^{-1} \mathbf{X}^{T} \mathbf{W}_{x}(\mathbf{X} \boldsymbol{\beta}+\mathbf{N f})\right] \\
& =\boldsymbol{\beta}+\left(\mathbf{X}^{T} \mathbf{W}_{x} \mathbf{X}\right)^{-1} \mathbf{X}^{T} \mathbf{W}_{x} \mathbf{N f}
\end{aligned}
$$

Note que ambos estimadores são viesados. Porém, podemos escolher $\lambda$ adequadamente de forma a diminuir o viés dos estimadores. Dado que a expressão (3.22) e a segunda parcela de (3.23) dependem de $\lambda$, percebe-se que à medida que diminuímos o valor desse parâmetro, menor o viés atribuído aos estimadores e, para $\lambda \downarrow 0, \mathrm{E}(\widehat{\mathbf{f}}) \downarrow \mathbf{f}$ e $\mathrm{E}(\widehat{\boldsymbol{\beta}}) \downarrow \boldsymbol{\beta}$. 
Já as matrizes de variância-covariância de $\widehat{\mathbf{f}}$ e $\widehat{\boldsymbol{\beta}}$ são dadas, respectivamente, por

$$
\begin{aligned}
\operatorname{Var}(\widehat{\mathbf{f}}) & =\operatorname{Var}\left[\left(\mathbf{N}^{T} \mathbf{W}_{f} \mathbf{N}+\lambda \mathbf{K}\right)^{-1} \mathbf{N}^{T} \mathbf{W}_{f} \mathbf{y}\right] \\
& =\left[\left(\mathbf{N}^{T} \mathbf{W}_{f} \mathbf{N}+\lambda \mathbf{K}\right)^{-1} \mathbf{N}^{T} \mathbf{W}_{f}\right]^{T}\left(\mathbf{N}^{T} \mathbf{W}_{f} \mathbf{N}+\lambda \mathbf{K}\right)^{-1} \mathbf{N}^{T} \mathbf{W}_{f} \sigma^{2}
\end{aligned}
$$

e

$$
\begin{aligned}
\operatorname{Var}(\widehat{\boldsymbol{\beta}}) & =\operatorname{Var}\left[\left(\mathbf{X}^{T} \mathbf{W}_{x} \mathbf{X}\right)^{-1} \mathbf{X}^{T} \mathbf{W}_{x} \mathbf{y}\right] \\
& =\left[\left(\mathbf{X}^{T} \mathbf{W}_{x} \mathbf{X}\right)^{-1} \mathbf{X}^{T} \mathbf{W}_{x}\right]^{T}\left(\mathbf{X}^{T} \mathbf{W}_{x} \mathbf{X}\right)^{-1} \mathbf{X}^{T} \mathbf{W}_{x} \sigma^{2}
\end{aligned}
$$

\subsubsection{Erro padrão}

Sob os mesmos argumentos da Seção 2.6.2, podemos obter as matrizes de variância-covariância assintóticas das estimativas de máxima verossimilhança penalizadas pela inversa da informação de Fisher penalizada, ou seja,

$$
\widehat{\operatorname{Var}(\widehat{\boldsymbol{\theta}})} \approx \mathcal{I}_{p}^{-1}(\widehat{\boldsymbol{\theta}})
$$

em que

$$
\mathcal{I}_{p}^{-1}(\boldsymbol{\theta})=\left(\begin{array}{cc}
\mathcal{I}_{p}^{\beta \mathbf{f}^{-1}}(\boldsymbol{\theta}) & \mathbf{0} \\
\mathbf{0} & \mathcal{I}_{p}^{\sigma^{2} \sigma^{2}(\boldsymbol{\theta})}
\end{array}\right)
$$

e

$$
\mathcal{I}_{p}^{\beta \mathbf{f}^{-1}}(\boldsymbol{\theta})=\left(\begin{array}{cc}
\left(\mathbf{X}^{T} \mathbf{W}_{x} \mathbf{X}\right)^{-1} & -\mathbf{C} \\
-\mathbf{C}^{T} & \left(\mathbf{N}^{T} \mathbf{W}_{f} \mathbf{N}+\lambda \sigma^{2} \mathbf{K}\right)^{-1}
\end{array}\right),
$$

com $\mathbf{C}=\left(\mathbf{X}^{T} \mathbf{W X}\right)^{-1} \mathbf{X}^{T} \mathbf{W N}\left(\mathbf{N}^{T} \mathbf{W}_{f} \mathbf{N}+\lambda \sigma^{2} \mathbf{K}\right)^{-1}$. Logo, as matrizes de variância-covariância assintótica de $\widehat{\boldsymbol{\beta}}$ e $\widehat{\mathbf{f}}$ são dadas por

$$
\begin{aligned}
\widehat{\operatorname{Var}(\widehat{\boldsymbol{\beta}})} & \approx \mathcal{I}_{p}^{\boldsymbol{\beta}^{-1}}(\boldsymbol{\theta})=\left(\mathbf{X}^{T} \mathbf{W}_{x} \mathbf{X}\right)^{-1} \quad \mathrm{e} \\
\widehat{\operatorname{Var}(\widehat{\mathbf{f}})} & \approx \mathcal{I}_{p}^{\mathrm{ff}^{-1}}(\boldsymbol{\theta})=\left(\mathbf{N}^{T} \mathbf{W}_{f} \mathbf{N}+\lambda \sigma^{2} \mathbf{K}\right)^{-1}
\end{aligned}
$$

\subsection{Técnicas de diagnóstico}

A análise de diagnóstico é uma etapa importante na verificação das suposições iniciais do modelo e na verificação de observações com influência desproporcional no ajuste. Nesta seção trataremos de estudar as técnicas de diagnóstico existentes para os MLPs.

Assim como nos MANS, as técnicas de diagnóstico conhecidas na literatura (como análise de resíduos, pontos de alavanca, e distância de Cook) podem ser estendidas para o caso semiparamétrico, já que Eubank [1984] mostra que a matriz hat desses modelos, mesmo sem a propriedade de idempotência, converge para a matriz hat do modelo linear usual à medida que aumenta-se o valor do parâmetro de suavização. Além disso, Kim et al. [2002] verificaram que é possível analisar os resíduos, os pontos de alavanca e os pontos influentes isoladamente sob os componentes paramétri- 
cos e não paramétricos do modelo, já que constataram que uma observação pode ser influente na parte paramétrica e não ter influência na parte não paramétrica, e vice versa. Mais do que isso, ainda constataram que uma observação pode não ser influente na média, e ainda sim ser influente em parte dos parâmetros.

Dado esse cenário, neste trabalho estudaremos a análise de diagnóstico sob 3 enfoques: influência na parte paramétrica, influência na parte não paramétrica e influência na média.

\subsubsection{Decomposição da matriz $\overline{\mathbf{H}}(\lambda)$}

Como vimos na Seção 3.4.1, a matriz hat do MLP já tem expressão definida, porém ainda não foram definidas as matrizes hat referentes aos componentes paramétrico e não paramétrico. Nesta seção, trataremos de obter essas matrizes, baseadas na formulação de Kim et al. [2002]. Seja f definida em (3.13). Logo, o componente não paramétrico do MLP pode ser escrito como

$$
\mathbf{N f}=\mathbf{N}\left(\mathbf{N}^{T} \mathbf{W} \mathbf{N}+\lambda \mathbf{K}\right)^{-1} \mathbf{N}^{T} \mathbf{W}(\mathbf{y}-\mathbf{X} \boldsymbol{\beta})=\mathbf{H}(\lambda)(\mathbf{y}-\mathbf{X} \boldsymbol{\beta}),
$$

em que $\mathbf{H}(\lambda)$ é exatamente a matriz hat do MANS definida no Capítulo 2. Usando (3.27) em (3.12), obtemos o sistema $p \times p$ de equações lineares

$$
\mathbf{X}^{T} \mathbf{W}(\mathbf{I}-\mathbf{H}(\lambda)) \mathbf{X} \boldsymbol{\beta}=\mathbf{X}^{T} \mathbf{W}(\mathbf{I}-\mathbf{H}(\lambda)) \mathbf{y}
$$

Seguindo a notação de Kim et al. [2002], defina $\widetilde{\mathbf{X}}=(\mathbf{I}-\mathbf{H}(\lambda)) \mathbf{X}$ e $\widetilde{\mathbf{y}}=(\mathbf{I}-\mathbf{H}(\lambda)) \mathbf{y}$. Substituindo em (3.28) e fazendo algumas manipulações algébricas, temos que o estimador de $\boldsymbol{\beta}$ dado por $\widehat{\boldsymbol{\beta}}=$ $\left(\widetilde{\mathbf{X}}^{T} \mathbf{W} \widetilde{\mathbf{X}}\right)^{-1} \widetilde{\mathbf{X}}^{T} \mathbf{W} \widetilde{\mathbf{y}}$ e o componente paramétrico $\mathbf{X} \widehat{\boldsymbol{\beta}}$ expresso como

$$
\begin{aligned}
\mathbf{X} \widehat{\boldsymbol{\beta}} & =\mathbf{X}\left(\widetilde{\mathbf{X}}^{T} \mathbf{W} \widetilde{\mathbf{X}}\right)^{-1} \widetilde{\mathbf{X}}^{T} \mathbf{W} \widetilde{\mathbf{y}} \\
& =(\mathbf{I}-\mathbf{H}(\lambda))^{-1} \widetilde{\mathbf{X}}\left(\widetilde{\mathbf{X}}^{T} \mathbf{W} \widetilde{\mathbf{X}}\right)^{-1} \widetilde{\mathbf{X}}^{T} \mathbf{W}(\mathbf{I}-\mathbf{H}(\lambda)) \mathbf{y} \\
& =\widetilde{\mathbf{H}} \mathbf{y}
\end{aligned}
$$

Logo, o vetor de valores ajustados de $\mathbf{y}$ é dado por

$$
\begin{aligned}
\widehat{\mathbf{y}} & =\mathbf{X} \widehat{\boldsymbol{\beta}}+\mathbf{N} \widehat{\mathbf{f}} \\
& =\widetilde{\mathbf{H}} \mathbf{y}+\mathbf{H}(\lambda)(\mathbf{y}-\mathbf{X} \widehat{\boldsymbol{\beta}}) \\
& =[\widetilde{\mathbf{H}}+\mathbf{H}(\lambda)(\mathbf{I}-\widetilde{\mathbf{H}})] \mathbf{y} \\
& =\widetilde{\mathbf{H}}(\lambda) \mathbf{y} .
\end{aligned}
$$

Note que $\overline{\mathbf{H}}(\lambda)=\widetilde{\mathbf{H}}+\mathbf{H}^{*}(\lambda)$, em que $\widetilde{\mathbf{H}}$ representa a matriz hat referente ao componente paramétrico e $\mathbf{H}^{*}(\lambda)=\mathbf{H}(\lambda)(\mathbf{I}-\widetilde{\mathbf{H}})$ representa a matriz hat do componente não paramétrico do MLP. 


\subsubsection{Alavanca}

Da mesma forma que no MANS, podemos fazer a detecção de observações influentes na estimação dos parâmetros dos MLPs. Os pontos de alavanca podem exercer forte influência na variabilidade do estimador, e segundo Kim et al. [2002], podemos fazer a análise de pontos de alavanca observando a diagonal principal da matriz hat associada ao componente não paramétrico, ao componente paramétrico e à média.

Seja $\widetilde{h}_{i i}$ os elementos da diagonal principal da matriz hat associada ao componente paramétrico, $h_{i i}$ os elementos da diagonal principal da matriz hat associada ao componente não paramétrico e $\bar{h}_{i i}^{*}$ os elementos da diagonal principal da matriz hat associada à média. A detecção de altos valores de $\widetilde{h}_{i i}, h_{i i}^{*}$ ou $\bar{h}_{i i}$ indicam que a $i$-ésima observação pode ser um ponto de alavanca, ou seja, podem exercer influência desproporcional em sua própria predição.

\subsubsection{Resíduos}

A análise de resíduos é um dos procedimentos mais utilizados para avaliar se há afastamentos importantes das suposições feitas para o modelo e detectar pontos aberrantes. Os resíduos ordinários procuram medir a discrepância entre o valor observado e o ajustado de cada indivíduo. Por (3.16), sabemos que o vetor de valores esperados de $\mathbf{y}$ é dado por

$$
\widehat{\mathbf{y}}=\mathbf{X} \widehat{\boldsymbol{\beta}}+\mathbf{N} \widehat{\mathbf{f}}=\overline{\mathbf{H}}(\lambda) \mathbf{y}
$$

e consequentemente o vetor de resíduos ordinários pode ser expresso como

$$
\mathbf{r}=\mathbf{y}-\widehat{\mathbf{y}}=(\mathbf{I}-\overline{\mathbf{H}}(\lambda)) \mathbf{y}
$$

O vetor de resíduos $\mathbf{r}$ possui distribuição Normal com vetor de médias zero e matriz de variânciacovariância dada por

$$
\operatorname{Var}(\mathbf{r})=(\mathbf{I}-\overline{\mathbf{H}}(\lambda))^{T}(\mathbf{I}-\overline{\mathbf{H}}(\lambda)) \sigma^{2}
$$

Para melhor comparação entre os resíduos, consideraremos uma versão padronizada definida como

$$
\mathbf{r}^{*}=\frac{\mathbf{r}}{\sqrt{\widehat{\operatorname{Var}}(\mathbf{r})}}
$$

em que $\widehat{\operatorname{Var}}(\mathbf{r})$ é a diagonal principal da estimativa da matriz $\operatorname{Cov}(\mathbf{r})$ definida em (3.30).

\subsubsection{Influência}

Como medida para detecção de pontos influentes, usaremos a distância de Cook [1977] adaptada para o caso dos MLPs. Assim, como Kim et al. [2002], as análises de pontos de influência serão feitas sob 3 aspectos: análise de pontos de influência no componente paramétrico, no componente não paramétrico e na média. Valores altos dessas distâncias podem indicar alta influência da observação 
no ajuste do modelo e sua eliminação pode resultar em alterações nas interpretações dos modelos.

- Influência no componente paramétrico

A medida de influência da $i$-ésima observação em $\widehat{\boldsymbol{\beta}}$ é definida pela distância de Cook simplificada

$$
\widetilde{D}_{i}=\frac{\widetilde{r}_{i}^{2} \widetilde{h}_{i i}}{s^{2}\left(1-\widetilde{h}_{i i}\right)^{2} \operatorname{tr}\{\widetilde{\mathbf{H}}\}},
$$

em que $\widetilde{r}_{i}$ é o $i$-ésimo componente do vetor de resíduos $\widetilde{\mathbf{r}}, \operatorname{com} \widetilde{\mathbf{r}}=(\mathbf{I}-\widetilde{\mathbf{H}}) \widetilde{\mathbf{y}}, \widetilde{h}_{i i}$ é o $i$-ésimo componente da diagonal de $\widetilde{\mathbf{H}}$ e $s^{2}$ é o estimador não viesado de $\sigma^{2}$. Note que $\operatorname{tr}\{\widetilde{\mathbf{H}}\}=p$ e podemos reescrever (3.32) como

$$
\widetilde{D}_{i}=\frac{\widetilde{r}_{i}^{2} \widetilde{h}_{i i}}{s^{2}\left(1-\widetilde{h}_{i i}\right)^{2} p} .
$$

- Influência no componente não paramétrico

A medida de influência da $i$-ésima observação em $\widehat{\mathbf{f}}$ é definida pela distância de Cook simplificada

$$
D_{i}^{*}=\frac{\left(r_{i}^{*} h_{i i}^{*}\right)^{2}}{s^{2}\left(1-h_{i i}^{*}\right)^{2} \operatorname{tr}\left\{\mathbf{H}^{*}(\lambda)\right\}}
$$

em que $r_{i}^{*}$ é o $i$-ésimo componente do vetor de resíduos $\mathbf{r}^{*}, \operatorname{com} \mathbf{r}^{*}=\left(\mathbf{I}-\mathbf{H}^{*}(\lambda)\right) \mathbf{y}, h_{i i}^{*}$ é o $i$-ésimo componente da diagonal de $\mathbf{H}^{*}(\lambda)$ e $s^{2}$ é o estimador não viesado de $\sigma^{2}$.

- Influência na média

A medida de influência da $i$-ésima observação no vetor de valores ajustados pode ser expressa, similarmente, de forma simplificada dada por

$$
\bar{D}_{i}=\frac{\bar{r}_{i}^{2} \bar{h}_{i i}}{s^{2}\left(1-\bar{h}_{i i}\right)^{2} \operatorname{tr}\{\overline{\mathbf{H}}(\lambda)\}},
$$

que é equivalente à distância de Cook do modelo linear clássico.

\subsection{Seleção de modelos}

Em analogia ao MANS, foram adaptados os critérios de Akaike [1974] utilizando o desvio global penalizado baseado no logaritmo da função de verossimilhança penalizada, criando os critérios de informação de Akaike penalizado $\left(\mathrm{AIC}_{p}\right)$ e o critério de informação de Schwarz penalizado $\left(\mathrm{SIC}_{p}\right)$. A ideia desses critérios é priorizar modelos com menor número de parâmetros com o acréscimo de funções ao desvio global penalizado. Os critérios $\mathrm{AIC}_{p}$ e $\mathrm{SIC}_{p}$ ficam dados por

$$
\mathrm{AIC}_{p}=\mathrm{DG}_{p}+2 \operatorname{tr}\{\overline{\mathbf{H}}(\lambda)\} \quad \mathrm{e}
$$




$$
\mathrm{SIC}_{p}=\mathrm{DG}_{p}+\operatorname{tr}\{\overline{\mathbf{H}}(\lambda)\} \log n
$$

em que $\mathrm{DG}_{p}=-2 \ell_{p}(\boldsymbol{\theta}, \lambda)$, com $\ell_{p}(\boldsymbol{\theta}, \lambda)$ sendo o logaritmo da função de verossimilhança penalizada para o estimador de $\boldsymbol{\theta}, \operatorname{tr}\{\overline{\mathbf{H}}(\lambda)\}$ correspondem aos graus de liberdade do ajuste e $n$ é o total de observações na amostra. Note que $\operatorname{tr}\{\overline{\mathbf{H}}\}=\operatorname{tr}\{\widetilde{\mathbf{H}}\}+\operatorname{tr}\left\{\mathbf{H}^{*}\right\}=p+\operatorname{tr}\left\{\mathbf{H}^{*}\right\}$, ou seja, podemos reescrever os critérios (3.36) e (3.37) como

$$
\begin{gathered}
\mathrm{AIC}_{p}=\mathrm{DG}_{p}+2 p+2 \operatorname{tr}\left\{\mathbf{H}^{*}\right\} \\
\mathrm{SIC}_{p}=\mathrm{DG}_{p}+p \log n+\operatorname{tr}\left\{\mathbf{H}^{*}\right\} \log n .
\end{gathered}
$$

Nos dois critérios sugeridos, maximizar a função de verossimilhança penalizada é equivalente a minimizar o critério de seleção, ou seja, dentre os modelos considerados, devemos escolher aquele que possui o menor valor para esses critérios.

\subsection{Considerações}

Este capítulo teve como principal objetivo estudar toda a teoria referente aos modelos lineares parciais normais na literatura, como a estimação dos componentes paramétricos e não paramétrico pela maximização da verossimilhança penalizada através do método direto e via algoritmo backfitting, estimação do parâmetro de suavização e as medidas de diagnóstico utilizadas para verificação da adequabilidade do ajuste, como as medidas da diagonal principal das matrizes hat dos componentes paramétricos, não paramétrico e para a média para detecção de possíveis pontos de alavanca e pontos influentes. O estudo dos resíduos ordinários e resíduos ordinários padronizados também foram estudados com o objetivo de avaliar as suposições para o modelo via gráfico normal de probabilidades com envelope gerado e a avaliação de indícios de heteroscedasticidade quando gerado o gráfico desses resíduos contra os valores ajustados pelo modelo. 


\section{Capítulo 4}

\section{Aplicações}

Neste capítulo apresentamos duas aplicações referentes aos modelos apresentados anteriormente, sendo um dos conjuntos de dados obtido do livro de Montgomery et al. [2001] com ajuste de uma função não paramétrica via gamlss; a outra aplicação é uma revisão do ajuste realizado aos dados sobre snacks da Seção 1.1 supondo um MLP com a variável semanas considerada como não paramétrica e ajuste realizado via gam.

\subsection{MANS: Saída elétrica}

Nesta seção apresentamos uma aplicação dos tópicos discutidos no Capítulo 2, com o objetivo de ilustrar, principalmente, a estimação dos parâmetros e as técnicas de diagnóstico no MANS. A aplicação considera um conjunto de dados descrito em Montgomery et al. [2001] e encontra-se no Apêndice A.

Um engenheiro deseja investigar a relação entre a velocidade do vento e o valor de saída elétrica de um moinho. A Figura 4.1 mostra o diagrama de dispersão em que podemos perceber que a depêndencia entre essas variáveis pode ser representada de uma forma não linear.

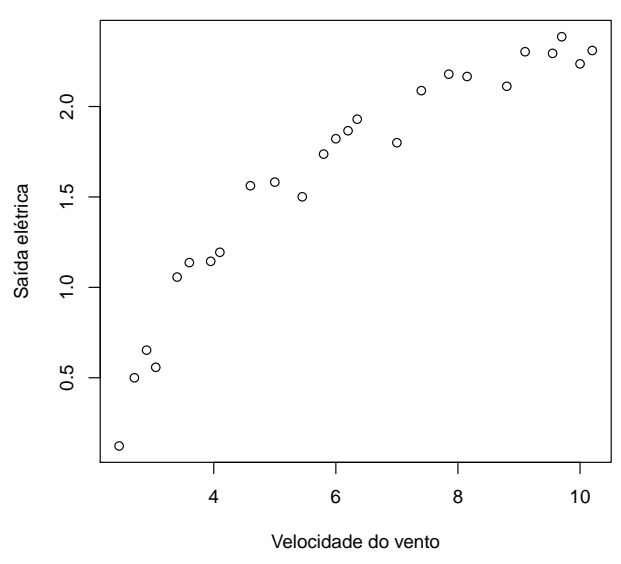

Figura 4.1: Diagrama de dispersão entre a saída elétrica e a velocidade do vento. 


\subsubsection{O modelo}

Assumindo que a saída elétrica possui distribuição normal e a variável saída elétrica possui comportamento não linear, vamos propor um modelo em que a velocidade do vento é tratada de forma não paramétrica ajustado via spline cúbico, escrito como

$$
y_{i}=f\left(t_{i}\right)+\epsilon_{i}
$$

em que $y_{i}$ é o valor da saída elétrica da $i$-ésima observação, $i=1, \ldots, 25, t_{i}$ é o valor da $i$-ésima velocidade do vento, $f(\cdot)$ é uma função não paramétrica arbitrária que depende da velocidade do vento e $\epsilon_{i}$ é um erro aleatório. Matricialmente, o modelo pode ser escrito como

$$
\mathbf{y}=\mathbf{N f}+\boldsymbol{\epsilon}
$$

sendo $\mathbf{y}$ o vetor aleatório $(25 \times 1)$ com as 25 saídas elétricas observadas, $\mathbf{N}$ é a matriz de incidência $(25 \times 25)$ que corresponde à matriz identidade, $\mathbf{f}$ é o vetor $(25 \times 1)$ cujos componentes correspondem à avaliação funcional $f(\cdot)$ nos valores da variável explicativa referente à velocidade do vento, e $\boldsymbol{\epsilon}=\left(\epsilon_{1}, . ., \epsilon_{25}\right)^{T}$ é o vetor de erros aleatórios.

\subsubsection{Ajuste do MANS}

Os modelos foram ajustados via gamlss no R, utilizando a função de verossimilhança penalizada sob erros normais, e utilizando o critério de informação de Schwarz escolhemos os graus de liberdade que minimizassem esse critério. A Tabela 4.1 mostra o comportamento dos valores estimados da verossimilhança penalizada e do critério de informação de Schwarz à medida que aumentamos os graus de liberdade.

Tabela 4.1: Valores estimados do logaritmo da função de verossimilhança penalizada e do critério de informação de Schwarz para diferentes graus de liberdade para os dados de saída elétrica.

\begin{tabular}{lrr}
\hline GLE & $-2 \ell_{p}$ & SIC \\
\hline 2 & $-3,32$ & 3,11 \\
3 & $-18,72$ & $-9,07$ \\
4 & $-33,04$ & $-20,17$ \\
5 & $-40,89$ & $-24,80$ \\
$\mathbf{6}$ & $\mathbf{- 4 5 , 6 1}$ & $\mathbf{- 2 6 , 2 9}$ \\
7 & $-48,68$ & $-26,15$ \\
8 & $-51,01$ & $-25,26$ \\
9 & $-53,10$ & $-24,13$ \\
10 & $-55,17$ & $-22,98$ \\
11 & $-57,32$ & $-21,91$ \\
12 & $-59,57$ & $-20,94$ \\
\hline
\end{tabular}

Note que a utilização dos valores estimados da função de verossimilhança penalizada não parece ser um bom critério para a escolha dos graus de liberdade associados ao ajuste, já que o desvio 
global penalizado diminui à medida que aumentamos os graus de liberdade. O modelo escolhido talvez seja um modelo com pouca variância e maior viés. A Figura 4.2 ilustra esse comportamento, em que modelos ajustados para graus de liberdades mais elevados possuem curvas pouco suaves. Pela Tabela 4.1 e a Figura 4.3, sugerimos a escolha de 6 graus de liberdade, que possui desvio global penalizado -45, 61 e critério de informação de Schwarz -26, 29. O parâmetro de suavização obtido para o modelo escolhido é $\widehat{\lambda}=0,5947$.
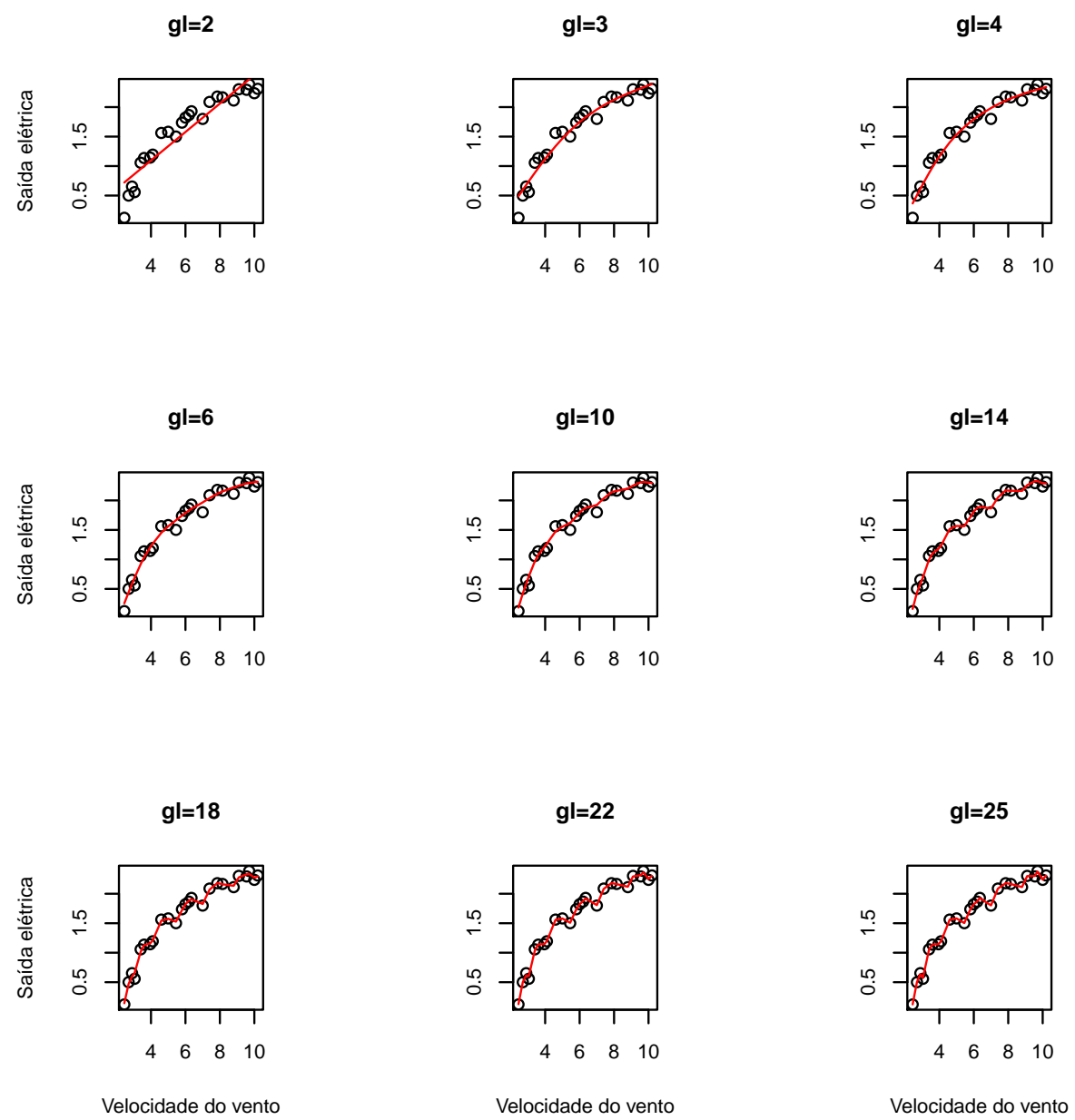

Figura 4.2: Gráficos dos modelos ajustados para diferentes graus de liberdade aos dados de saída elétrica.

As estimativas da função não paramétrica, do parâmetro de dispersão, e os respectivos erros padrão encontram-se na Tabela 4.2. Os erros padrão foram calculados através da inversa da matriz de informação de Fisher penalizada, e podemos perceber que o erro padrão associado a $\widehat{f\left(t_{1}\right)}$ é ligeiramente maior do que os demais.

\subsubsection{Diagnóstico e ajustes adicionais}

Para o modelo ajustado construímos o gráfico normal de probabilidades para o resíduo ordinário padronizado com envelope gerado, bem como alguns gráficos adicionais de diagnóstico, incluindo os gráficos de pontos de alavanca, pontos influentes e dois gráficos de análise dos resíduos. Conforme 


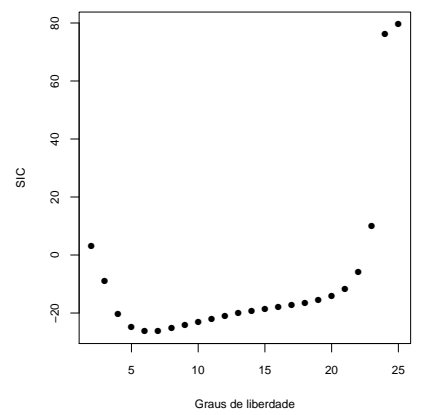

Figura 4.3: Gráficos dos valores obtidos para o critério de informação de Schwarz à medida que aumentamos os graus de liberdade do ajuste para os dados de saída elétrica.

Tabela 4.2: Estimativas e erros padrão da função não paramétrica e do parâmetro de dispersão para o modelo ajustado aos dados de saída elétrica.

\begin{tabular}{lrrr}
\hline$\#$ & $f(\cdot)$ & Estimativa & Erro padrão \\
\hline 1 & $f\left(t_{1}\right)$ & 0,254 & 0,062 \\
2 & $f\left(t_{2}\right)$ & 0,441 & 0,028 \\
3 & $f\left(t_{3}\right)$ & 0,586 & 0,025 \\
4 & $f\left(t_{4}\right)$ & 0,691 & 0,027 \\
5 & $f\left(t_{5}\right)$ & 0,918 & 0,029 \\
6 & $f\left(t_{6}\right)$ & 1,032 & 0,029 \\
7 & $f\left(t_{7}\right)$ & 1,203 & 0,029 \\
8 & $f\left(t_{8}\right)$ & 1,268 & 0,032 \\
9 & $f\left(t_{9}\right)$ & 1,451 & 0,040 \\
10 & $f\left(t_{10}\right)$ & 1,561 & 0,040 \\
11 & $f\left(t_{11}\right)$ & 1,664 & 0,037 \\
12 & $f\left(t_{12}\right)$ & 1,743 & 0,028 \\
13 & $f\left(t_{13}\right)$ & 1,788 & 0,025 \\
14 & $f\left(t_{14}\right)$ & 1,829 & 0,026 \\
15 & $f\left(t_{15}\right)$ & 1,857 & 0,032 \\
16 & $f\left(t_{16}\right)$ & 1,966 & 0,045 \\
17 & $f\left(t_{17}\right)$ & 2,039 & 0,040 \\
18 & $f\left(t_{18}\right)$ & 2,113 & 0,037 \\
19 & $f\left(t_{19}\right)$ & 2,153 & 0,042 \\
20 & $f\left(t_{20}\right)$ & 2,221 & 0,042 \\
21 & $f\left(t_{21}\right)$ & 2,251 & 0,037 \\
22 & $f\left(t_{22}\right)$ & 2,287 & 0,031 \\
23 & $f\left(t_{23}\right)$ & 2,296 & 0,029 \\
24 & $f\left(t_{24}\right)$ & 2,309 & 0,031 \\
25 & $f\left(t_{25}\right)$ & 2,317 & 0,059 \\
& $\log (\sigma)$ & $-2,434$ & 0,184 \\
\hline & & &
\end{tabular}

visto na Figura 4.4, parece não haver afastamentos importantes da suposição de normalidade para os erros uma vez que grande parte dos resíduos estão dentro da banda de confiança no gráfico normal 
de probabilidades com envelope gerado. Porém, a tendência não aleatória dos resíduos dentro da banda pode sugerir outro tipo de função não paramétrica. Já na Figura 4.5, é possível observar que a observação \#1 é destacada como um possível ponto influente, provavelmente porque o valor da saída elétrica dessa observação é considerado baixo dada a velocidade do vento. Dessa forma, foi realizado um novo ajuste desconsiderando essa observação, e as estimativas dos parâmetros para esse modelo encontram-se na Tabela 4.3.

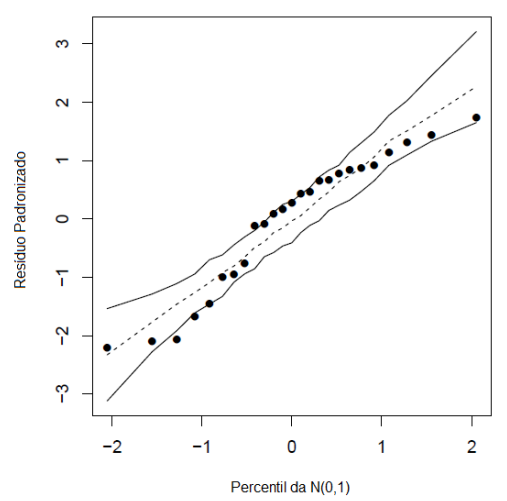

Figura 4.4: Gráfico normal de probabilidades com envelope gerado para o resíduo ordinário padronizado referente ao ajuste do MANS para os dados de saída elétrica.
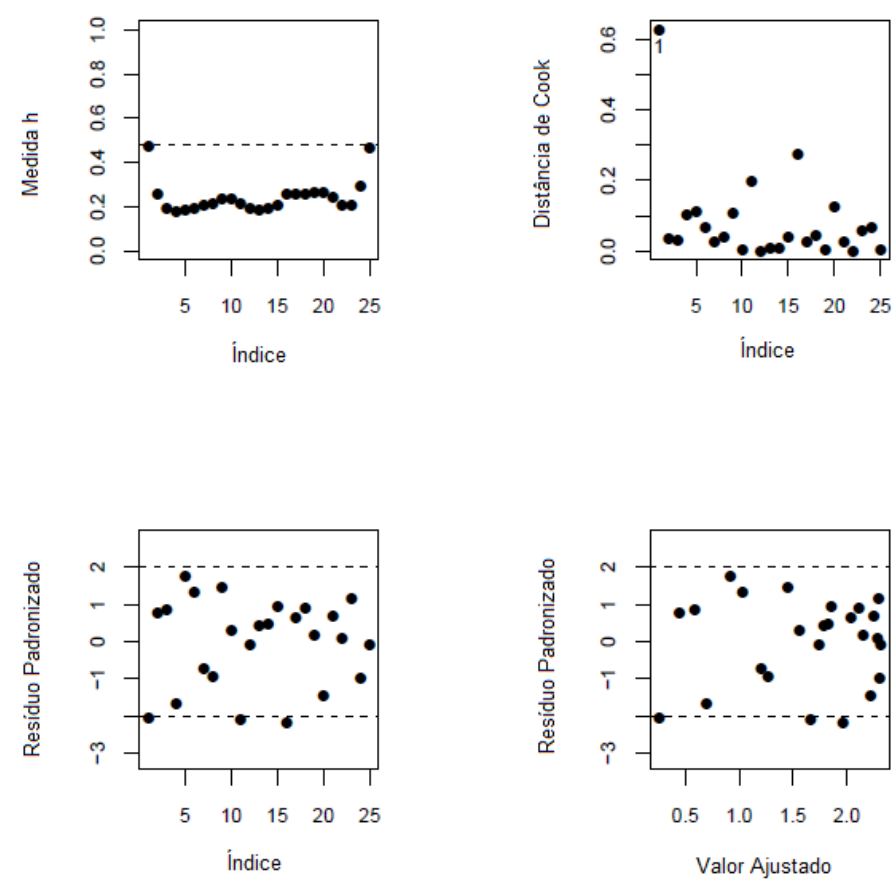

Figura 4.5: Gráficos de diagnóstico referentes ao MANS ajustado aos dados de saída elétrica.

Comparando as Tabelas 4.2 e 4.3, nota-se que não há indícios de diferenças significativas entre as estimativas de $f(\cdot)$ e nem a diminuição dos erros padrão associados. Além disso, o gráfico normal 
Tabela 4.3: Estimativas e erros padrão da função não paramétrica e do parâmetro de dispersão para o modelo ajustado aos dados de saída elétrica sem a observação \#1.

\begin{tabular}{lrrr}
\hline$\#$ & $f(\cdot)$ & Estimativa & Erro padrão \\
\hline 2 & $f\left(t_{2}\right)$ & 0,515 & 0,055 \\
3 & $f\left(t_{3}\right)$ & 0,634 & 0,027 \\
4 & $f\left(t_{4}\right)$ & 0,724 & 0,026 \\
5 & $f\left(t_{5}\right)$ & 0,927 & 0,029 \\
6 & $f\left(t_{6}\right)$ & 1,033 & 0,029 \\
7 & $f\left(t_{7}\right)$ & 1,196 & 0,028 \\
8 & $f\left(t_{8}\right)$ & 1,260 & 0,031 \\
9 & $f\left(t_{9}\right)$ & 1,444 & 0,040 \\
10 & $f\left(t_{10}\right)$ & 1,556 & 0,039 \\
11 & $f\left(t_{11}\right)$ & 1,660 & 0,036 \\
12 & $f\left(t_{12}\right)$ & 1,742 & 0,027 \\
13 & $f\left(t_{13}\right)$ & 1,787 & 0,024 \\
14 & $f\left(t_{14}\right)$ & 1,829 & 0,025 \\
15 & $f\left(t_{15}\right)$ & 1,857 & 0,031 \\
16 & $f\left(t_{16}\right)$ & 1,966 & 0,044 \\
17 & $f\left(t_{17}\right)$ & 2,037 & 0,039 \\
18 & $f\left(t_{18}\right)$ & 2,114 & 0,037 \\
19 & $f\left(t_{19}\right)$ & 2,154 & 0,041 \\
20 & $f\left(t_{20}\right)$ & 2,221 & 0,041 \\
21 & $f\left(t_{21}\right)$ & 2,252 & 0,036 \\
22 & $f\left(t_{22}\right)$ & 2,288 & 0,030 \\
23 & $f\left(t_{23}\right)$ & 2,296 & 0,028 \\
24 & $f\left(t_{24}\right)$ & 2,309 & 0,030 \\
25 & $f\left(t_{25}\right)$ & 2,315 & 0,057 \\
& $\log (\sigma)$ & $-2,466$ & 0,183 \\
\hline & & &
\end{tabular}

Tabela 4.4: Comparação do desvio global e critérios de informação para o modelo completo e o modelo sem a observação \#1 para os dados de saída elétrica.

\begin{tabular}{lrr}
\hline & Modelo completo & Modelo sem obs. \#1 \\
\hline$-2 \ell_{p}(\boldsymbol{\theta})$ & $-45,61$ & $-47,09$ \\
$\mathrm{AIC}_{p}$ & $-33,60$ & $-35,09$ \\
$\mathrm{SIC}_{p}$ & $-26,29$ & $-27,77$ \\
\hline
\end{tabular}

de probabilidades com envelope gerado (Figura 4.6) e os gráficos de diagnóstico (Figura 4.7) não apresentam mudanças importantes quando comparados com os gráficos do modelo considerando todas as observações. Portanto, o ajuste considerando todas as observações parece ser o mais adequado. Na Figura 4.8 temos o gráfico do ajuste final do MANS considerando todas as observações, que parece adequado. 


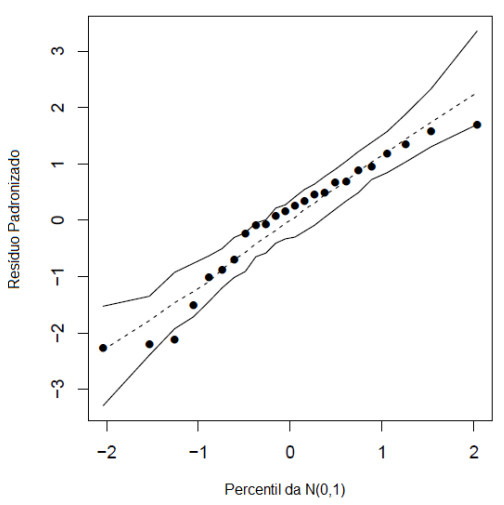

Figura 4.6: Gráfico normal de probabilidades com envelope gerado para o resíduo ordinário padronizado referente ao ajuste do MANS para os dados de saída elétrica sem a observação \#1.
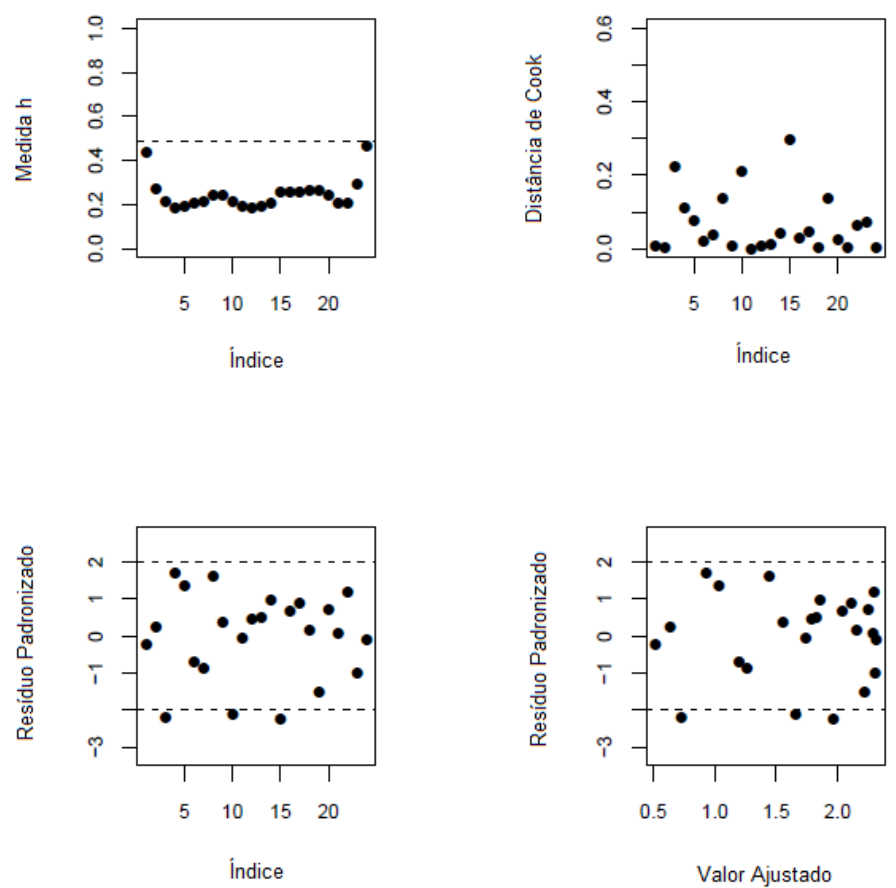

Figura 4.7: Gráficos de diagnóstico referentes ao MANS ajustado aos dados de saída elétrica sem a observação \#1.

\subsection{MLP: Snacks}

Voltando ao exemplo motivacional apresentado na Seção 1.1, ajustamos um modelo linear parcial em que a variável semanas é introduzida no modelo como um componente não paramétrico. Seja MP o modelo ajustado de forma paramétrica na Seção 1.1, e MLP o modelo linear parcial com erros normais proposto no Capítulo 3 . 


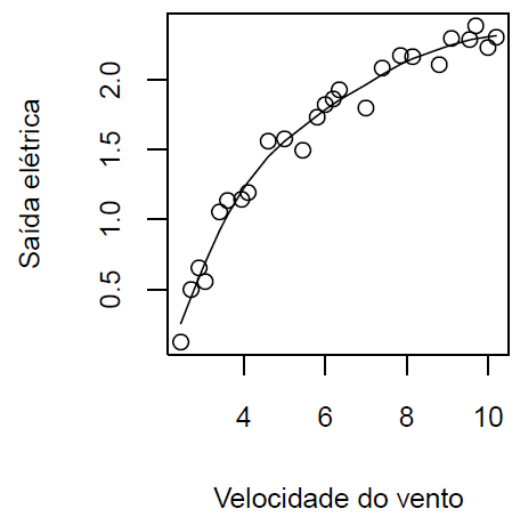

Figura 4.8: MANS ajustado aos dados de saída elétrica.

\subsubsection{O modelo}

Seja $y_{i j k}$ o valor do logaritmo da força de cisalhamento associada à $k$-ésima unidade experimental do $i$-ésimo tipo de snack na $j$-ésima semana, em que $i=1(A), 2(B), 3(C), 4(D), 5(E), j=2,4, \ldots, 20$ e $k=1, \ldots, 15$. Vamos propor o modelo linear parcial

$$
y_{i j k}=\beta_{0}+\beta_{i}+f\left(t_{i}\right)+\epsilon_{i j k}
$$

em que $\beta_{1}=0$, de modo que $\beta_{0}, \beta_{0}+\beta_{2}, \beta_{0}+\beta_{3}, \beta_{0}+\beta_{4}$ e $\beta_{0}+\beta_{5}$ denotam respectivamente os efeitos dos snacks A, B, C, D e E, controlados pela semana através da função $f\left(t_{j}\right)$ com $t_{1}=2, t_{2}=$ $4, \ldots, t_{10}=20$. Assumimos que $\epsilon_{i j k}$ são erros mutuamente independentes tais que $\epsilon_{i j k} \sim N\left(0, \sigma^{2}\right)$.

Matricialmente podemos escrever o modelo dado em (4.1) na seguinte forma:

$$
\mathrm{y}=\mathrm{X} \boldsymbol{\beta}+\mathbf{N f}+\boldsymbol{\epsilon}
$$

em que $\mathbf{X}$ é uma matriz $(750 \times 5)$ tal que $\mathbf{X}=\left[\mathbf{X}_{1}^{T}, \ldots, \mathbf{X}_{10}^{T}\right]^{T}$ com $\mathbf{X}_{j}$ de dimensão $(75 \times 5)$ e $\mathbf{X}_{1}=\mathbf{X}_{2}=\ldots=\mathbf{X}_{10}$ dada por $\mathbf{X}_{j}=\left(\mathbf{A}_{1}^{T}, \mathbf{A}_{2}^{T}, \mathbf{A}_{3}^{T}, \mathbf{A}_{4}^{T}, \mathbf{A}_{5}^{T}\right)^{T}$ sendo $\mathbf{A}_{1}$ sendo uma matriz $(15 \times 5)$ de zeros com 1's na primeira coluna e $\mathbf{A}_{j}$ uma matriz $(15 \times 5)$ de zeros com 1 's na primeira e na $j$-ésima colunas, $j=2,3,4,5$. Além disso, $\mathbf{N}$ é a matriz de incidência $(750 \times 10)$ dada por $\mathbf{N}=\left[\mathbf{N}_{1}^{T}, \ldots, \mathbf{N}_{10}^{T}\right]^{T}$ em que $\mathbf{N}_{j}$ é uma matriz de dimensão $(75 \times 10)$ de zeros com 1 's na $j$-ésima coluna, e $\epsilon$ é um vetor de erros de dimensão $(750 \times 1)$. Portanto, os parâmetros a serem estimados são dados por $\boldsymbol{\beta}=\left(\beta_{0}, \beta_{2}, \beta_{3}, \beta_{4}, \beta_{5}\right)^{T}$ e $\mathbf{f}=\left(f_{2}, f_{4}, \ldots, f_{20}\right)^{T}$.

\subsubsection{Ajuste do MLP}

A estimação do parâmetro de suavização $\hat{\lambda}$ foi realizada pela validação cruzada generalizada sob o modelo proposto, e o resultado é $\hat{\lambda}=0,236$. Os erros padrão das estimativas dos componentes paramétricos e não paramétricos foram obtidos através da inversa da informação de Fisher penalizada, e os resultados dos dois ajustes são apresentados na Tabela 4.5.

Nota-se na Tabela 4.5 que a estimativa do intercepto do modelo MLP é maior que o do modelo 
Tabela 4.5: Estimativas de máxima verossimilhança penalizada dos efeitos dos tipos de snacks com os respectivos erros padrão aproximados para os modelos MP e MLP ajustados as dados sobre snacks.

\begin{tabular}{lrrrr}
\hline & Modelo MP & & Modelo MLP & \\
Efeito & Estimativa & Erro Padrão & Estimativa & Erro Padrão \\
\hline Intercepto & 3,805 & 0,036 & 4,150 & 0,018 \\
Grupo A & 0,000 & - & 0,000 & - \\
Grupo B & $-0,166$ & 0,027 & $-0,166$ & 0,025 \\
Grupo C & $-0,076$ & 0,027 & $-0,076$ & 0,025 \\
Grupo D & $-0,240$ & 0,027 & $-0,240$ & 0,025 \\
Grupo E & $-0,257$ & 0,027 & $-0,257$ & 0,025 \\
\hline
\end{tabular}

MP. Com relação as estimativas referentes aos grupos, nota-se que o tipo A é o snack com maior textura média (na escala logaritmica), seguido do tipo $\mathrm{C}$ e $\mathrm{B}$, respectivamente. Os tipos D e E parecem não diferir significativamente e possuem em média menor textura. Essas tendências valem para ambos os modelos, mostrando que o controle da semana através da forma paramétrica ou não paramétrica não muda as interpretações inferenciais dos efeitos dos tipos de snacks na textura média. Contudo, o controle através da função não paramétrica reduz um pouco as estimativas dos erros padrão. A estimativa do parâmetro de dispersão $\left(\widehat{\sigma}^{2}=0,050\right)$ também altera pouco quando comparado com o modelo linear usual $\left(\widehat{\sigma}^{2}=0,052\right)$.

Na Tabela 4.6 temos as estimativas dos parâmetros referentes ao componente não paramétrico, em que podemos observar que a $14^{a}$ e a $16^{a}$ semanas são as que possuem maior acréscimo na força necessária para o cisalhamento, e que os erros padrão associados aos parâmetros são bastante semelhantes. Já na Figura 4.9, temos os gráficos de perfis ajustados dos modelos MP e MLP, em que podemos notar uma melhora das estimativas quando comparado com o gráfico de perfil de médias observado (Figura 1.1), principalmente nas primeiras semanas, além das semanas em que foram observadas altas médias de força necessária para o cisalhamento.

Tabela 4.6: Estimativas de máxima verossimilhança penalizadas com os respectivos erros padrão aproximados dos componentes não paramétricos referentes ao modelo MLP ajustados aos dados sobre snacks.

\begin{tabular}{lrr}
\hline Efeito & Estimativa & Erro Padrão \\
\hline Semana 2 & $-0,105$ & 0,026 \\
Semana 4 & $-0,219$ & 0,026 \\
Semana 6 & $-0,134$ & 0,026 \\
Semana 8 & $-0,023$ & 0,026 \\
Semana 10 & 0,060 & 0,026 \\
Semana 12 & 0,041 & 0,026 \\
Semana 14 & 0,216 & 0,026 \\
Semana 16 & 0,151 & 0,026 \\
Semana 18 & 0,081 & 0,026 \\
Semana 20 & $-0,069$ & 0,026 \\
\hline
\end{tabular}



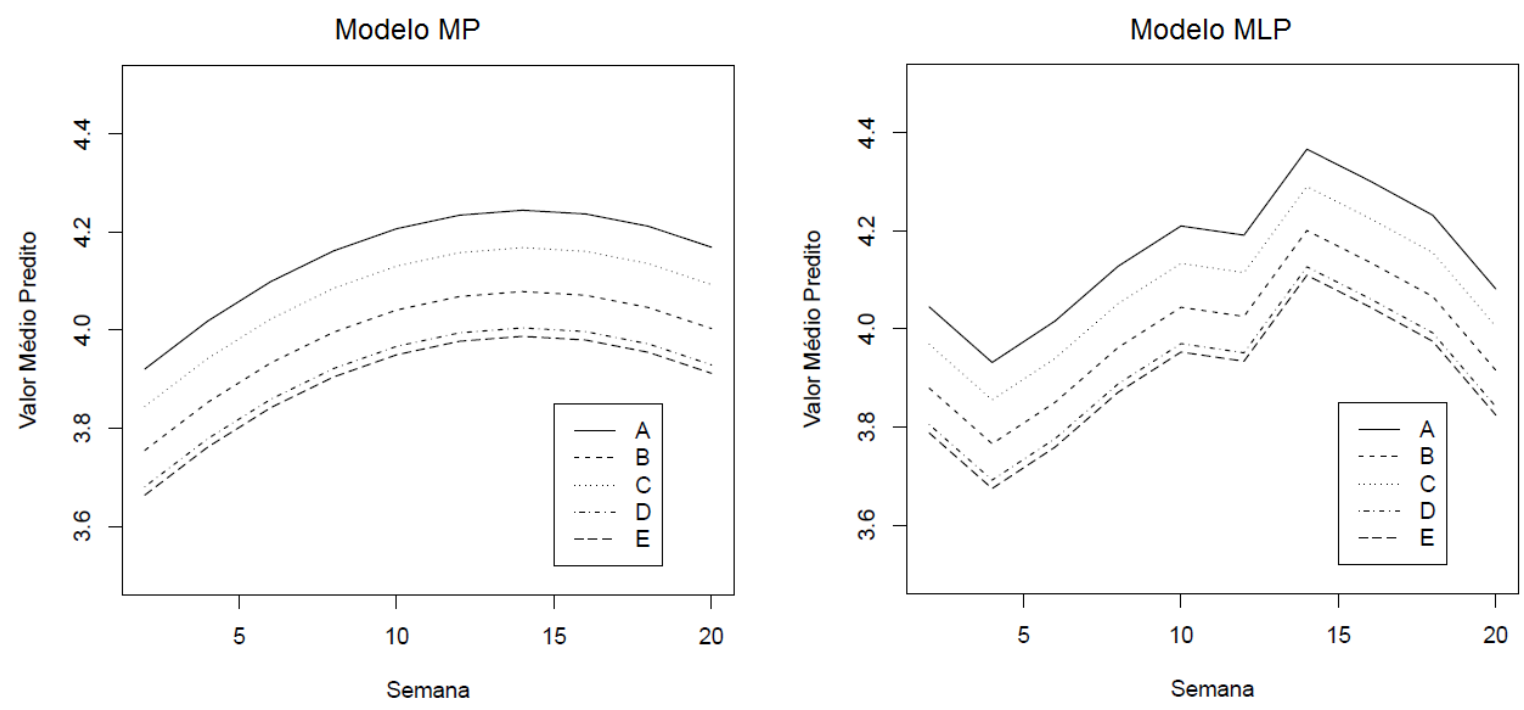

Figura 4.9: Perfis ajustados dos modelos MP e MLP referentes aos dados sobre snacks.

\subsubsection{Diagnóstico e ajustes adicionais}

Para o modelo ajustado construímos o gráfico normal de probabilidades para o resíduo ordinário padronizado com envelope gerado e gráficos adicionais de diagnóstico, incluindo os gráficos de pontos de alavanca, pontos influentes e dois gráficos de análise dos resíduos. Conforme visto na Figura 4.10, o modelo parece bem ajustado uma vez que a maioria dos resíduos estão dentro da banda de confiança, com exceção de uma das extremidades, o que pode sugerir uma distribuição com caudas mais pesadas.

Na Figura 4.11, é possível observar que a observação \#452 aparece como possível ponto influente. Esse ponto pertence ao grupo de composição A, foi analisado na $14^{a}$ semana e possui força de cisalhamento muito menor do que a força das demais amostras observadas nesse grupo e na semana em questão. Dessa forma, foi realizado um novo ajuste desconsiderando essa observação, e as estimativas dos parâmetros para esse modelo encontram-se na Tabela 4.7. Já as estimativas dos GLEs e parâmetros de suavização do modelo completo e do modelo sem a observação \#452 encontram-se na Tabela 4.8.

Nota-se pela Tabela 4.7 que existe uma diferença pequena nas estimativas dos modelos, e que as maiores diferenças são vistas nas estimativas dos parâmetros relativos aos grupos de composição dos snacks e na $14^{a}$ semana. As estimativas dos parâmetros relativos à $14^{a}$ semana também possuem maior alteração quando comparadas com as diferenças das demais semanas. Com relação aos erros padrão relativos aos parâmetros, não há grandes diferenças entre as estimativas.

Os gráficos de diagnóstico também foram gerados para o modelo que desconsidera a observação \#452. Nota-se na Figura 4.12 que o modelo também tem problemas em uma das extremidades, porém grande parte dos pontos ainda estão dentro da banda de confiança. Já na Figura 4.13, não há indícios de pontos de alavanca e influentes. Dada a pouca diferença entre as estimativas dos dois modelos propostos, o modelo completo parece o mais adequado. 


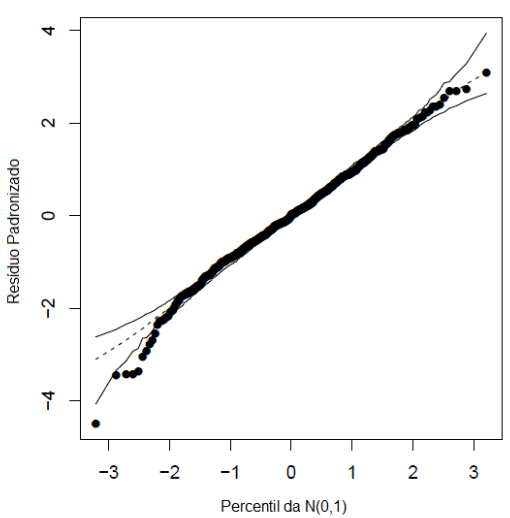

Figura 4.10: Gráfico normal de probabilidades com envelope gerado para o resíduo ordinário padronizado referente ao ajuste do MLP aos dados sobre snacks.
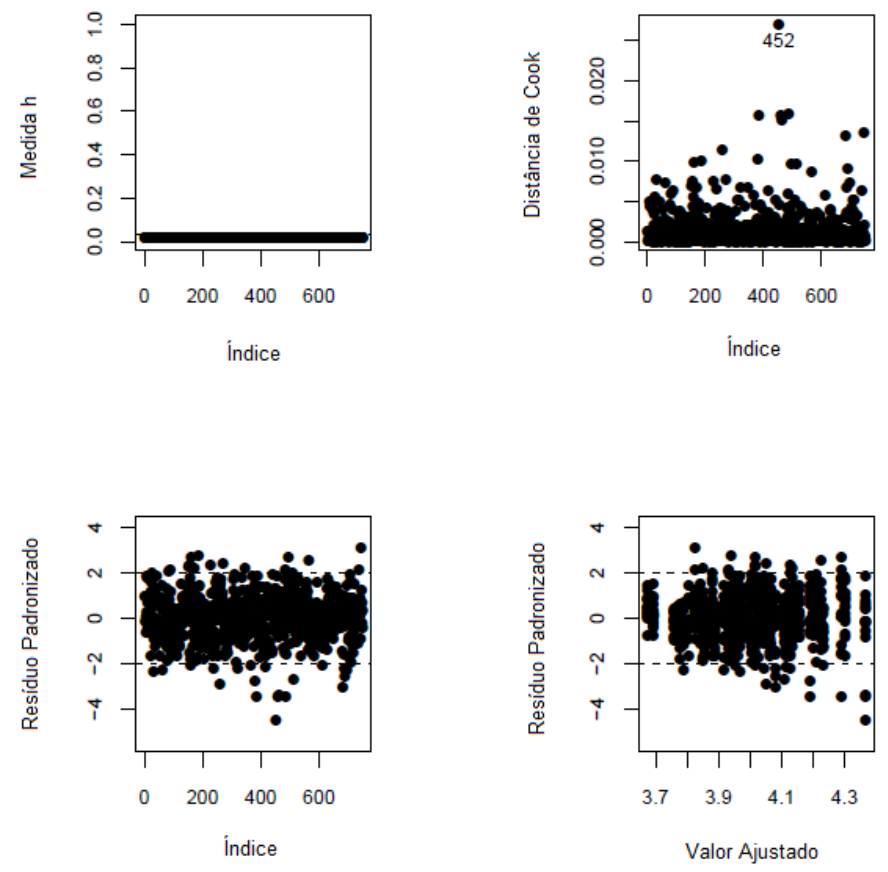

Figura 4.11: Gráficos de diagnóstico referentes ao modelo MLP ajustado aos dados sobre snacks. 
Tabela 4.7: Comparação entre as estimativas do MLP completo e do MLP sem a observação \#452 no ajuste aos dados sobre snacks.

\begin{tabular}{lrrrr}
\hline & Modelo MLP & & Modelo MLP sem \#452 & \\
Efeito & Estimativa & Erro Padrão & Estimativa & Erro Padrão \\
\hline Intercepto & 4,150 & 0,018 & 4,157 & 0,018 \\
Grupo A & $-0,000$ & - & 0,000 & - \\
Grupo B & $-0,166$ & 0,025 & 0,173 & 0,026 \\
Grupo C & $-0,076$ & 0,025 & $-0,083$ & 0,026 \\
Grupo D & $-0,240$ & 0,025 & $-0,247$ & 0,026 \\
Grupo E & $-0,257$ & 0,025 & $-0,264$ & 0,026 \\
Semana 2 & $-0,105$ & 0,026 & $-0,106$ & 0,026 \\
Semana 4 & $-0,219$ & 0,026 & $-0,221$ & 0,026 \\
Semana 6 & $-0,134$ & 0,026 & $-0,135$ & 0,026 \\
Semana 8 & $-0,023$ & 0,026 & $-0,024$ & 0,026 \\
Semana 10 & 0,060 & 0,026 & 0,060 & 0,026 \\
Semana 12 & 0,041 & 0,026 & 0,038 & 0,026 \\
Semana 14 & 0,216 & 0,026 & 0,230 & 0,026 \\
Semana 16 & 0,151 & 0,026 & 0,150 & 0,026 \\
Semana 18 & 0,081 & 0,026 & 0,081 & 0,026 \\
Semana 20 & $-0,069$ & 0,026 & $-0,070$ & 0,026 \\
\hline
\end{tabular}

Tabela 4.8: Comparação entre os GLEs, parâmetro de suavização e parâmetro de dispersão dos MLPs completo e sem a observação \#452 no ajuste aos dados sobre snacks.

\begin{tabular}{lrr}
\hline & Modelo completo & Modelo sem a observação \#452 \\
\hline GLE & 13,386 & 13,496 \\
$\hat{\lambda}$ & 0,236 & 0,188 \\
$\widehat{\sigma}^{2}$ & 0.050 & 0.049 \\
\hline
\end{tabular}

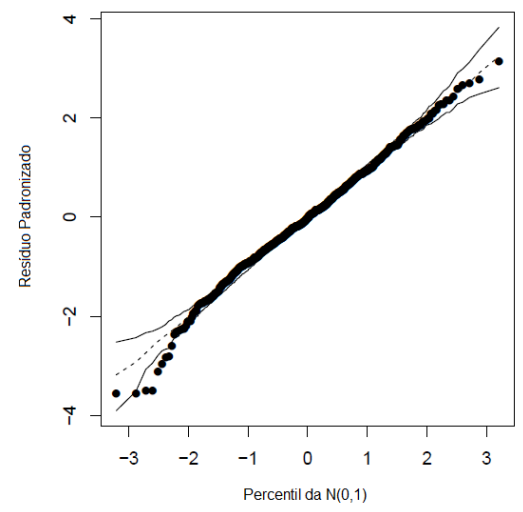

Figura 4.12: Gráfico normal de probabilidades com envelope gerado para o resíduo ordinário padronizado referente ao ajuste do MLP sem a observação \#452 ajustado aos dados sobre snacks. 

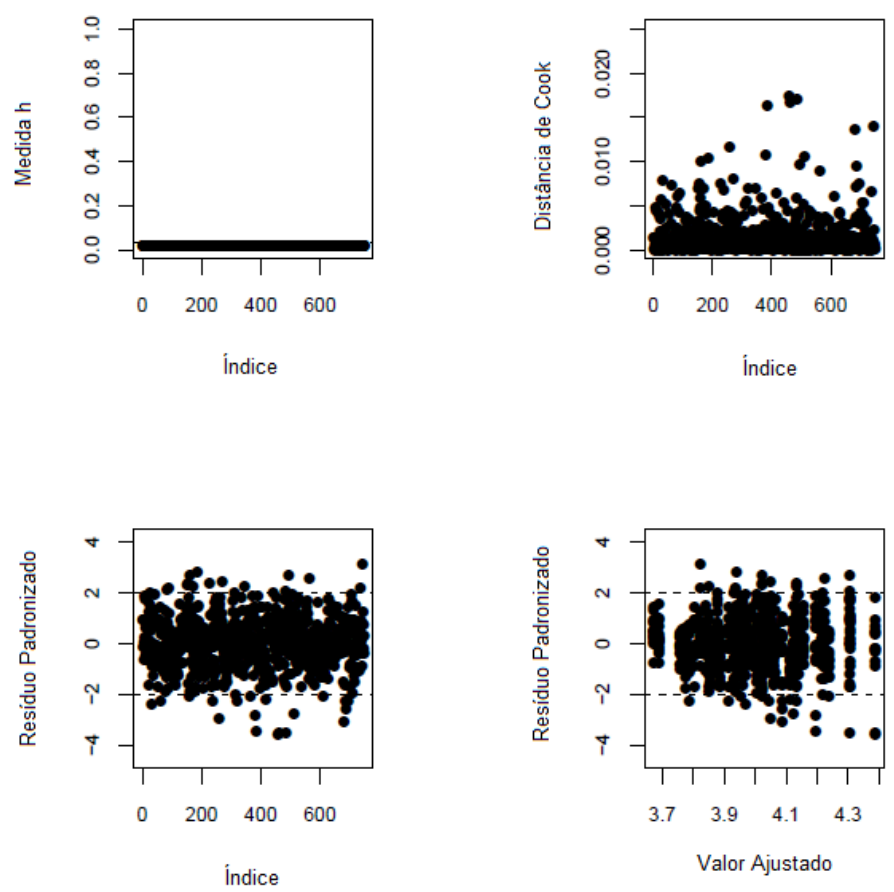

Figura 4.13: Gráficos de diagnóstico referentes ao modelo MLP sem a observação \#452 ajustado aos dados sobre snacks . 


\section{Capítulo 5}

\section{Conclusões}

\subsection{Considerações finais}

Nesta dissertação apresentamos os modelos aditivos normais simples e os modelos semiparamétricos sob erros normais, com o objetivo de detalhar toda a teoria referente a esses modelos na literatura. Foram utilizados splines cúbicos para o ajuste da função não paramétrica e foram aplicadas funções de verossimilhança penalizadas para a obtenção dos estimadores de máxima verossimilhança com os respectivos erros padrão aproximados. Foi realizado também um estudo referente às distribuições assintóticas dos estimadores de máxima verossimilhança e testes de hipóteses, porém ainda existem poucos estudos inferenciais para esses modelos.

Sabendo que os valores esperados podem ser escritos como uma combinação linear da variável resposta, foram obtidas as matrizes projetoras dos MANSs para dados ajustados via gamlss, e derivadas as propriedades dessa matriz e da matriz projetora do MLP com o objetivo de utilizá-las como ferramenta na análise de diagnóstico. Gráficos de diagnóstico também foram adaptados para avaliar a adequabilidade dos modelos propostos.

Do ponto de vista prático, vimos que os MANSs são facilmente interpretáveis visualmente através dos gráficos da função não paramétrica versus variável resposta, e que a rugosidade da curva depende dos graus de liberdade e do parâmetro de suavização. O modelo pareceu adequado quando ajustado aos dados de saída elétrica, porém houve indícios de que outro tipo de função não paramétrica poderia ser uma melhor escolha pela não aleatoriedade dos resíduos. Já na aplicação referente ao MLP, a estimativa dos interceptos dos modelos MP e MLP apresentaram uma diferença maior quando comparados com os parâmetros referentes aos grupos de composição, além dos erros padrão associados a esses estimadores apresentarem valores menores no modelo com componente não paramétrica. Os gráficos de perfis dos modelos MP e MLP ajustados mostraram um ganho no ajuste quando considerado a variável semana não parametricamente. Gráficos de diagnósticos foram adaptados para ambas as aplicações e foram constatados alguns pontos influentes, que quando retirados não alteraram significativamente as estimativas dos modelos. 


\subsection{Implementação computacional}

Os códigos desenvolvidos ao longo desta dissertação para a estimação nos MANSs via pacote gamlss no $\mathrm{R}$ e os gráficos de adequabilidade dos modelos, gráficos de diagnóstico e gráficos de resíduos dos MANSs e MLPs ajustados via gamlss e gam, respectivamenter, estão disponíveis no Apêndice D.

\subsection{Sugestões para pesquisas futuras}

Dentre as sugestões para pesquisas futuras, podemos destacar o estudo dos MANSs e MLPs normais heteroscedásticos, que ocorre quando a suposição de igualdade de variâncias não é satisfeita. Outra sugestão seria estudar os modelos aditivos simples e modelos lineares parciais com outras distribuições além da normal.

Além das análises de dignóstico realizadas neste trabalho, uma sugestão de pesquisa futura é estudar a influência local para os dois modelos estudados.

Dada a carência de estudos no assunto, uma possibilidade seria estudar a inferência dos estimadores no contexto dos modelos com componentes não paramétricos, derivações de intervalos de confiança e testes de hipóteses. Podemos estudar também os modelos aditivos normais, em que todas as variáveis explicativas são ajustadas não parametricamente. Por fim, outra sugestão é estudar o ajuste do componente não paramétrico considerando outras funções que não os splines cúbicos, como por exemplo, as B-splines e $\mathrm{P}$-splines. 


\section{Apêndice A}

\section{Dados Saída elétrica}

Neste apêndice, apresentamos os dados das velocidades do vento e das saídas elétricas na Tabela A.1.

Tabela A.1: Dados de saída elétrica.

\begin{tabular}{ccc}
\hline$i$ & Velocidade vento $(\mathrm{mph}), t_{i}$ & DC Output, $y_{i}$ \\
\hline 1 & 2,45 & 0,123 \\
2 & 2,70 & 0,500 \\
3 & 2,90 & 0,653 \\
4 & 3,05 & 0,558 \\
5 & 3,40 & 1,057 \\
6 & 3,60 & 1,137 \\
7 & 3,95 & 1,144 \\
8 & 4,10 & 1,194 \\
9 & 4,60 & 1,562 \\
10 & 5,00 & 1,582 \\
11 & 5,45 & 1,501 \\
12 & 5,80 & 1,737 \\
13 & 6,00 & 1,822 \\
14 & 6,20 & 1,866 \\
15 & 6,35 & 1,930 \\
16 & 7,00 & 1,800 \\
17 & 7,40 & 2,088 \\
18 & 7,85 & 2,179 \\
19 & 8,15 & 2,166 \\
20 & 8,80 & 2,112 \\
21 & 9,10 & 2,303 \\
22 & 9,55 & 2,294 \\
23 & 9,70 & 2,386 \\
24 & 10,00 & 2,236 \\
25 & 10,20 & 2,310 \\
\hline
\end{tabular}


APÊNDICE A 


\section{Apêndice B}

\section{Derivações do logaritmo da função de verossimilhança penalizada no MANS}

Neste apêndice são apresentados detalhes dos cálculos necessários para obter as funções escore e informações de Fisher para o MANS. Para maiores detalhes sobre álgebra e diferenciação com matrizes, consultar Searle [1982].

Como visto na Seção (2.4.2), o logaritmo da função de verossimilhança penalizada do MANS (2.1) é dado por

$$
\ell_{p}(\boldsymbol{\theta}, \lambda)=-\frac{n}{2} \log 2 \pi \sigma^{2}-\frac{1}{2 \sigma^{2}}(\mathbf{y}-\mathbf{N f})^{T}(\mathbf{y}-\mathbf{N f})-\frac{1}{2} \lambda \mathbf{f}^{T} \mathbf{K f},
$$

sendo $\boldsymbol{\theta}=\left(\mathbf{f}^{T}, \sigma^{2}\right)^{T}$ o vetor de parâmetros $(r \times 1)$ a serem estimados através da maximização do logaritmo da função de verossimilhança penalizada. Para todos os cálculos realizados a seguir, estamos assumindo que (B.1) obedece às condições de regularidade e que todas as derivadas parciais com relação à $\mathbf{f}$ e $\sigma^{2}$ existem.

\section{B.1 Derivadas de primeira ordem}

Derivando (B.1) em relação ao vetor não paramétrico f, obtemos o vetor $(r \times 1)$ das funções escores penalizadas $\mathbf{U}_{p}^{\mathbf{f}}(\boldsymbol{\theta})$, dado por

$$
\begin{aligned}
\mathbf{U}_{p}^{\mathbf{f}}(\boldsymbol{\theta})=\frac{\partial \ell_{p}(\boldsymbol{\theta}, \lambda)}{\partial \mathbf{f}} & =\frac{\partial}{\partial \mathbf{f}}\left[-\frac{1}{2 \sigma^{2}}(\mathbf{y}-\mathbf{N f})^{T}(\mathbf{y}-\mathbf{N f})-\frac{1}{2} \lambda \mathbf{f}^{T} \mathbf{K f}\right] \\
& =-\frac{1}{2} \frac{\partial}{\partial \mathbf{f}}\left[\sigma^{-2}\left(\mathbf{y}^{T} \mathbf{y}-2 \mathbf{f}^{T} \mathbf{N}^{T} \mathbf{y}+\mathbf{f}^{T} \mathbf{N}^{T} \mathbf{N f}\right)+\lambda \mathbf{f}^{T} \mathbf{K f}\right] \\
& =-\frac{1}{2}\left[-2 \sigma^{-2} \mathbf{N}^{T} \mathbf{y}+2 \sigma^{-2} \mathbf{N}^{T} \mathbf{N f}+2 \lambda \mathbf{K f}\right] \\
& =\sigma^{-2} \mathbf{N}^{T}(\mathbf{y}-\mathbf{N f})-\lambda \mathbf{K f} .
\end{aligned}
$$


Com relação ao parâmetro de dispersão, a função escore penalizada $\mathrm{U}_{p}^{\sigma^{2}}(\boldsymbol{\theta})$ fica dada por

$$
\begin{aligned}
\mathrm{U}_{p}^{\sigma^{2}}(\boldsymbol{\theta})=\frac{\partial \ell_{p}(\boldsymbol{\theta}, \lambda)}{\partial \sigma^{2}} & =\frac{\partial}{\partial \sigma^{2}}\left[-\frac{n}{2} \log 2 \pi \sigma^{2}-\frac{1}{2 \sigma^{2}}(\mathbf{y}-\mathbf{N f})^{T}(\mathbf{y}-\mathbf{N f})\right] \\
& =-\frac{n}{2 \sigma^{2}}+\frac{(\mathbf{y}-\mathbf{N f})^{T}(\mathbf{y}-\mathbf{N f})}{2 \sigma^{4}} \\
& =\frac{1}{2 \sigma^{2}}\left[\frac{(\mathbf{y}-\mathbf{N f})^{T}(\mathbf{y}-\mathbf{N f})}{\sigma^{2}}-n\right]
\end{aligned}
$$

\section{B.2 Derivadas de segunda ordem}

Derivando a expressão (B.2) com relação ao efeito não paramétrico, temos a segunda derivada do logaritmo da função de verossimilhança penalizada com relação a f dada por

$$
\begin{aligned}
\frac{\partial^{2} \ell_{p}(\boldsymbol{\theta}, \lambda)}{\partial \mathbf{f} \partial \mathbf{f}^{T}}=\frac{\partial \mathbf{U}_{p}^{\mathbf{f}}(\boldsymbol{\theta})}{\partial \mathbf{f}^{T}} & =\frac{\partial}{\partial \mathbf{f}^{T}}\left[\sigma^{-2} \mathbf{N}^{T}(\mathbf{y}-\mathbf{N f})-\lambda \mathbf{K} \mathbf{f}\right] \\
& =-\sigma^{-2} \mathbf{N}^{T} \mathbf{N}-\lambda \mathbf{K} \\
& =-\left(\sigma^{-2} \mathbf{N}^{T} \mathbf{N}+\lambda \mathbf{K}\right)
\end{aligned}
$$

que implica na informação de Fisher penalizada

$$
\mathcal{I}_{p}^{\mathrm{ff}}(\boldsymbol{\theta})=-\mathrm{E}\left(\frac{\partial^{2} \ell_{p}(\boldsymbol{\theta}, \lambda)}{\partial \mathbf{f} \partial \mathbf{f} f^{T}}\right)=\sigma^{-2} \mathbf{N}^{T} \mathbf{N}+\lambda \mathbf{K}
$$

Já a derivada de (B.2) com relação à $\sigma^{2}$ fica dada por

$$
\begin{aligned}
\frac{\partial^{2} \ell_{p}(\boldsymbol{\theta}, \lambda)}{\partial \mathbf{f} \partial \sigma^{2}}=\frac{\partial \mathbf{U}_{p}^{\mathbf{f}}(\boldsymbol{\theta})}{\partial \sigma^{2}} & =\frac{\partial}{\partial \sigma^{2}}\left[\sigma^{-2} \mathbf{N}^{T}(\mathbf{y}-\mathbf{N f})-\lambda \mathbf{K f}\right] \\
& =-\sigma^{-4} \mathbf{N}^{T}(\mathbf{y}-\mathbf{N f})
\end{aligned}
$$

que implica na informação de Fisher penalizada

$$
\mathcal{I}_{p}^{\mathbf{f} \sigma^{2}}(\boldsymbol{\theta})=-\mathrm{E}\left(\frac{\partial^{2} \ell_{p}(\boldsymbol{\theta}, \lambda)}{\partial \mathbf{f} \partial \sigma^{2}}\right)=\sigma^{-4} \mathbf{N}^{T} \mathrm{E}(\mathbf{y}-\mathbf{N f})=\mathbf{0} .
$$

Com relação ao parâmetro de dispersão, temos que a derivada de (B.3) é expressa como

$$
\begin{aligned}
\frac{\partial^{2} \ell_{p}(\boldsymbol{\theta}, \lambda)}{\partial \sigma^{4}}=\frac{\partial \mathrm{U}_{p}^{\sigma^{2}}(\boldsymbol{\theta})}{\partial \sigma^{2}} & =\frac{\partial}{\partial \sigma^{2}}\left[\frac{1}{2 \sigma^{2}}\left(\frac{(\mathbf{y}-\mathbf{N f})^{T}(\mathbf{y}-\mathbf{N f})}{\sigma^{2}}-n\right)\right] \\
& =\frac{1}{\sigma^{4}}\left[\frac{n}{2}-\frac{(\mathbf{y}-\mathbf{N f})^{T}(\mathbf{y}-\mathbf{N f})}{\sigma^{2}}\right]
\end{aligned}
$$

que implica na informação de Fisher penalizada

$$
\mathcal{I}_{p}^{\sigma^{2} \sigma^{2}}(\boldsymbol{\theta})=-\mathrm{E}\left(\frac{\partial^{2} \ell_{p}(\boldsymbol{\theta}, \lambda)}{\partial \sigma^{4}}\right)=\frac{1}{\sigma^{4}}\left[\frac{\mathrm{E}\left[(\mathbf{y}-\mathbf{N f})^{T}(\mathbf{y}-\mathbf{N f})\right]}{\sigma^{2}}-\frac{n}{2}\right]=\frac{1}{\sigma^{4}}\left(n-\frac{n}{2}\right)=\frac{n}{2 \sigma^{4}}
$$


Já a derivada de (B.3) em relação à f fica dada por

$$
\begin{aligned}
\frac{\partial^{2} \ell_{p}(\boldsymbol{\theta}, \lambda)}{\partial \sigma^{2} \partial \mathbf{f}}=\frac{\partial \mathbf{U}_{p}^{\sigma^{2}}(\boldsymbol{\theta})}{\partial \mathbf{f}} & =\frac{\partial}{\partial \mathbf{f}}\left[\frac{(\mathbf{y}-\mathbf{N f})^{T}(\mathbf{y}-\mathbf{N f})}{2 \sigma^{4}}\right] \\
& =-\sigma^{-4} \mathbf{N}^{T}(\mathbf{y}-\mathbf{N f})
\end{aligned}
$$

que implica na informação de Fisher penalizada

$$
\mathcal{I}_{p}^{\sigma^{2} \mathbf{f}}(\boldsymbol{\theta})=-\mathrm{E}\left(\frac{\partial^{2} \ell_{p}(\boldsymbol{\theta}, \lambda)}{\partial \sigma^{2} \partial \mathbf{f}}\right)=\sigma^{-4} \mathbf{N}^{T} \mathrm{E}(\mathbf{y}-\mathbf{N f})=\mathbf{0} .
$$


64 APÊNDICE B 


\section{Apêndice C}

\section{Derivações do logaritmo da função de verossimilhança penalizada no MLP}

Neste apêndice são apresentados detalhes dos cálculos necessários para obter as funções escore e informações de Fisher para o MLP. Para maiores detalhes sobre álgebra e diferenciação com matrizes, consultar Searle [1982].

Como visto na Seção (2.4.2), o logaritmo da função de verossimilhança penalizada do MLP é dado por

$$
\ell_{p}(\boldsymbol{\theta}, \lambda)=-\frac{n}{2} \log 2 \pi \sigma^{2}-\frac{1}{2 \sigma^{2}}(\mathbf{y}-\mathbf{X} \boldsymbol{\beta}-\mathbf{N f})^{T} \mathbf{W}(\mathbf{y}-\mathbf{X} \boldsymbol{\beta}-\mathbf{N f})-\frac{1}{2} \lambda \mathbf{f}^{T} \mathbf{K f},
$$

sendo $\boldsymbol{\theta}=\left(\boldsymbol{\beta}^{T}, \mathbf{f}^{T}, \sigma^{2}\right)^{T}$ o vetor $(p+r+2 \times 1)$ de parâmetros a serem estimados via maximização do logaritmo da função de verossimilhança penalizada. Para todos os cálculos realizados a seguir, estamos assumindo que (C.1) obedece às condições de regularidade e que todas as derivadas parciais com relação à $\boldsymbol{\beta}, \mathbf{f}$ e $\sigma^{2}$ existem.

\section{C.1 Derivadas de primeira ordem}

Calculando a derivada de (C.1) em relação a $\boldsymbol{\beta}$, temos o vetor $(p+1 \times 1)$ de funções escore penalizadas dos efeitos paramétricos $\mathbf{U}_{p}^{\boldsymbol{\beta}}(\boldsymbol{\theta})$ dada por

$$
\begin{aligned}
\mathbf{U}_{p}^{\boldsymbol{\beta}}(\boldsymbol{\theta})=\frac{\partial \ell_{p}(\boldsymbol{\theta}, \lambda)}{\partial \boldsymbol{\beta}} & =\frac{\partial}{\partial \boldsymbol{\beta}}\left[-\frac{1}{2 \sigma^{2}}(\mathbf{y}-\mathbf{X} \boldsymbol{\beta}-\mathbf{N f})^{T} \mathbf{W}(\mathbf{y}-\mathbf{X} \boldsymbol{\beta}-\mathbf{N f})-\frac{1}{2} \lambda \mathbf{f}^{T} \mathbf{K f}\right] \\
& =-\frac{1}{2 \sigma^{2}} \frac{\partial}{\partial \boldsymbol{\beta}}\left[-2 \boldsymbol{\beta}^{T} \mathbf{X}^{T} \mathbf{W} \mathbf{y}+2 \boldsymbol{\beta}^{T} \mathbf{X}^{T} \mathbf{W N f}+\boldsymbol{\beta}^{T} \mathbf{X}^{T} \mathbf{W X} \boldsymbol{\beta}\right] \\
& =-\frac{1}{\sigma^{2}}\left(\mathbf{X}^{T} \mathbf{W} \mathbf{y}-\mathbf{X}^{T} \mathbf{W N f}-\mathbf{X}^{T} \mathbf{W} \mathbf{X} \boldsymbol{\beta}\right) \\
& =\sigma^{-2} \mathbf{X}^{T} \mathbf{W}(\mathbf{y}-\mathbf{X} \boldsymbol{\beta}-\mathbf{N f}) .
\end{aligned}
$$

Com relação ao vetor $(r \times 1)$ de efeito não paramétrico $\mathbf{f}$, temos a função escore penalizada 
$\mathbf{U}_{p}^{\mathbf{f}}(\boldsymbol{\theta})$ dada por

$$
\begin{aligned}
\mathbf{U}_{p}^{\mathbf{f}}(\boldsymbol{\theta})=\frac{\partial \ell_{p}(\boldsymbol{\theta}, \lambda)}{\partial \mathbf{f}} & =\frac{\partial}{\partial \mathbf{f}}\left[-\frac{1}{2 \sigma^{2}}(\mathbf{y}-\mathbf{X} \boldsymbol{\beta}-\mathbf{N} \mathbf{f})^{T} \mathbf{W}(\mathbf{y}-\mathbf{X} \boldsymbol{\beta}-\mathbf{N f})-\frac{1}{2} \lambda \mathbf{f}^{T} \mathbf{K f}\right] \\
& =-\frac{1}{2} \frac{\partial}{\partial \mathbf{f}}\left[\sigma^{-2}\left(-2 \mathbf{f}^{T} \mathbf{N}^{T} \mathbf{W} \mathbf{y}+2 \mathbf{f}^{T} \mathbf{N}^{T} \mathbf{W} \mathbf{X} \boldsymbol{\beta}+\mathbf{f}^{T} \mathbf{N}^{T} \mathbf{W} \mathbf{N f}\right)+\lambda \mathbf{f}^{T} \mathbf{K f}\right] \\
& =\sigma^{-2}\left(\mathbf{N}^{T} \mathbf{W} \mathbf{y}-\mathbf{N}^{T} \mathbf{W} \mathbf{X} \boldsymbol{\beta}-\mathbf{N}^{T} \mathbf{W} \mathbf{N f}\right)-\lambda \mathbf{K f} \\
& =\sigma^{-2} \mathbf{N}^{T} \mathbf{W}(\mathbf{y}-\mathbf{X} \boldsymbol{\beta}-\mathbf{N f})-\lambda \mathbf{K f} .
\end{aligned}
$$

Por fim, para o parâmetro de dispersão $\sigma^{2}$, temos a função escore penalizada $\mathrm{U}_{p}^{\sigma^{2}}$ dada por

$$
\begin{aligned}
\mathrm{U}_{p}^{\sigma^{2}}(\boldsymbol{\theta})=\frac{\partial \ell_{p}(\boldsymbol{\theta}, \lambda)}{\partial \sigma^{2}} & =\frac{\partial}{\partial \sigma^{2}}\left[-\frac{n}{2} \log 2 \pi \sigma^{2}-\frac{1}{2 \sigma^{2}}(\mathbf{y}-\mathbf{X} \boldsymbol{\beta}-\mathbf{N f})^{T} \mathbf{W}(\mathbf{y}-\mathbf{X} \boldsymbol{\beta}-\mathbf{N f})\right] \\
& =-\frac{n}{2 \sigma^{2}}+\frac{(\mathbf{y}-\mathbf{X} \boldsymbol{\beta}-\mathbf{N f})^{T} \mathbf{W}(\mathbf{y}-\mathbf{X} \boldsymbol{\beta}-\mathbf{N f})}{2 \sigma^{4}} \\
& =\frac{1}{2 \sigma^{2}}\left[\frac{(\mathbf{y}-\mathbf{X} \boldsymbol{\beta}-\mathbf{N f})^{T} \mathbf{W}(\mathbf{y}-\mathbf{X} \boldsymbol{\beta}-\mathbf{N f})}{\sigma^{2}}-n\right]
\end{aligned}
$$

\section{C.2 Derivadas de segunda ordem}

Calculando novamente a derivada de (C.2) com relação ao vetor $\boldsymbol{\beta}$, temos a segunda derivada do logaritmo da função de verossimilhança penalizada dada por

$$
\begin{aligned}
\frac{\partial^{2} \ell_{p}(\boldsymbol{\theta}, \lambda)}{\partial \boldsymbol{\beta} \partial \boldsymbol{\beta}^{T}}=\frac{\partial \mathbf{U}_{p}^{\boldsymbol{\beta}}(\boldsymbol{\theta})}{\partial \boldsymbol{\beta}^{T}} & =\frac{\partial}{\partial \boldsymbol{\beta}^{T}}\left[\sigma^{-2} \mathbf{X}^{T} \mathbf{W}(\mathbf{y}-\mathbf{X} \boldsymbol{\beta}-\mathbf{N f})\right] \\
& =-\sigma^{-2} \mathbf{X}^{T} \mathbf{W} \mathbf{X}
\end{aligned}
$$

que implica na informação de Fisher

$$
\mathcal{I}_{p}^{\boldsymbol{\beta} \boldsymbol{\beta}}(\boldsymbol{\theta})=-\mathrm{E}\left(\frac{\partial^{2} \ell_{p}(\boldsymbol{\theta}, \lambda)}{\partial \boldsymbol{\beta} \partial \boldsymbol{\beta}^{T}}\right)=\sigma^{-2} \mathbf{X}^{T} \mathbf{W X}
$$

Já a derivada de (C.2) em relação o vetor $\mathbf{f}$ fica dada por

$$
\begin{aligned}
\frac{\partial \mathbf{U}_{p}^{\boldsymbol{\beta}}(\boldsymbol{\theta})}{\partial \mathbf{f}^{T}}=\frac{\partial^{2} \ell_{p}(\boldsymbol{\theta}, \lambda)}{\partial \boldsymbol{\beta} \partial \mathbf{f}^{T}} & =\frac{\partial}{\partial \mathbf{f}^{T}}\left[\sigma^{-2} \mathbf{X}^{T} \mathbf{W}(\mathbf{y}-\mathbf{X} \boldsymbol{\beta}-\mathbf{N f})\right] \\
& =-\sigma^{-2} \mathbf{X}^{T} \mathbf{W} \mathbf{N}
\end{aligned}
$$

que implica na informação de Fisher

$$
\mathcal{I}_{p}^{\boldsymbol{\beta f}}(\boldsymbol{\theta})=-\mathrm{E}\left(\frac{\partial^{2} \ell_{p}(\boldsymbol{\theta}, \lambda)}{\partial \boldsymbol{\beta} \partial \mathbf{f}^{T}}\right)=\sigma^{-2} \mathbf{X}^{T} \mathbf{W N} .
$$


E, por fim, a derivada de (C.2) em relação a $\sigma^{2}$ fica dada por

$$
\begin{aligned}
\frac{\partial \mathbf{U}_{p}^{\boldsymbol{\beta}}(\boldsymbol{\theta})}{\partial \sigma^{2}}=\frac{\partial^{2} \ell_{p}(\boldsymbol{\theta}, \lambda)}{\partial \boldsymbol{\beta} \partial \sigma^{2}} & =\frac{\partial}{\partial \sigma^{2}}\left[\sigma^{-2} \mathbf{X}^{T} \mathbf{W}(\mathbf{y}-\mathbf{X} \boldsymbol{\beta}-\mathbf{N f})\right] \\
& =-\sigma^{-4} \mathbf{X}^{T} \mathbf{W}(\mathbf{y}-\mathbf{X} \boldsymbol{\beta}-\mathbf{N f})
\end{aligned}
$$

que implica na informação de Fisher

$$
\mathcal{I}_{p}^{\boldsymbol{\beta} \sigma^{2}}(\boldsymbol{\theta})=-\mathrm{E}\left(\frac{\partial^{2} \ell_{p}(\boldsymbol{\theta}, \lambda)}{\partial \boldsymbol{\beta} \partial \sigma^{2}}\right)=\sigma^{-4} \mathbf{X}^{T} \mathbf{W E}(\mathbf{y}-\mathbf{X} \boldsymbol{\beta}-\mathbf{N f})=\mathbf{0}
$$

Com relação ao vetor não paramétrico f, a segunda derivada do logaritmo da função de verossimilhança penalizada fica dada por

$$
\begin{aligned}
\frac{\partial^{2} \ell_{p}(\boldsymbol{\theta}, \lambda)}{\partial \mathbf{f} \partial \mathbf{f}^{T}}=\frac{\partial \mathbf{U}_{p}^{\mathbf{f}}(\boldsymbol{\theta})}{\partial \mathbf{f}^{T}} & =\frac{\partial}{\partial \mathbf{f}^{T}}\left[\sigma-2 \mathbf{N}^{T} \mathbf{W}(\mathbf{y}-\mathbf{X} \boldsymbol{\beta}-\mathbf{N f})-\lambda \mathbf{K f}\right] \\
& =-\sigma^{-2} \mathbf{N}^{T} \mathbf{W} \mathbf{N}-\lambda \mathbf{K} \\
& =-\left(\sigma^{-2} \mathbf{N}^{T} \mathbf{W} \mathbf{N}+\lambda \mathbf{K}\right),
\end{aligned}
$$

que implica na informação de Fisher

$$
\mathcal{I}_{p}^{\mathrm{ff}}(\boldsymbol{\theta})=-\mathrm{E}\left(\frac{\partial^{2} \ell_{p}(\boldsymbol{\theta}, \lambda)}{\partial \mathbf{f} \partial \mathbf{f}^{T}}\right)=\sigma^{-2} \mathbf{N}^{T} \mathbf{W} \mathbf{N}+\lambda \mathbf{K}
$$

Já a derivada de (C.3) em relação ao vetor $\boldsymbol{\beta}$ fica dada por

$$
\begin{aligned}
\frac{\partial \mathbf{U}_{p}^{\mathbf{f}}(\boldsymbol{\theta})}{\partial \boldsymbol{\beta}^{T}}=\frac{\partial^{2} \ell_{p}(\boldsymbol{\theta}, \lambda)}{\partial \mathbf{f} \partial \boldsymbol{\beta}^{T}} & =\frac{\partial}{\partial \boldsymbol{\beta}^{T}}\left[\sigma^{-2} \mathbf{N}^{T} \mathbf{W}(\mathbf{y}-\mathbf{X} \boldsymbol{\beta}-\mathbf{N f})\right] \\
& =-\sigma^{-2} \mathbf{N}^{T} \mathbf{W} \mathbf{X}
\end{aligned}
$$

que implica na informação de Fisher

$$
\mathcal{I}_{p}^{\mathbf{f} \boldsymbol{\beta}}(\boldsymbol{\theta})=-\mathrm{E}\left(\frac{\partial^{2} \ell_{p}(\boldsymbol{\theta}, \lambda)}{\partial \mathbf{f} \partial \boldsymbol{\beta}^{T}}\right)=\sigma^{-2} \mathbf{N}^{T} \mathbf{W} \mathbf{X}
$$

E, por fim, a derivada de (C.3) em relação a $\sigma^{2}$ fica dada por

$$
\begin{aligned}
\frac{\partial \mathbf{U}_{p}^{\mathbf{f}}(\boldsymbol{\theta})}{\partial \sigma^{2}}=\frac{\partial^{2} \ell_{p}(\boldsymbol{\theta}, \lambda)}{\partial \mathbf{f} \partial \sigma^{2}} & =\frac{\partial}{\partial \sigma^{2}}\left[\sigma^{-2} \mathbf{N}^{T} \mathbf{W}(\mathbf{y}-\mathbf{X} \boldsymbol{\beta}-\mathbf{N f})\right] \\
& =-\sigma^{-4} \mathbf{N}^{T} \mathbf{W}(\mathbf{y}-\mathbf{X} \boldsymbol{\beta}-\mathbf{N f})
\end{aligned}
$$

que implica na informação de Fisher

$$
\mathcal{I}_{p}^{\mathbf{f} \sigma^{2}}(\boldsymbol{\theta})=-\mathrm{E}\left(\frac{\partial^{2} \ell_{p}(\boldsymbol{\theta}, \lambda)}{\partial \mathbf{f} \partial \sigma^{2}}\right)=\sigma^{-4} \mathbf{N}^{T} \mathbf{W E}(\mathbf{y}-\mathbf{X} \boldsymbol{\beta}-\mathbf{N f})=\mathbf{0}
$$

Finalmente, com relação ao parâmetro de dispersão, temos a que a derivada de C.4 com relação 
a $\sigma^{2}$ fica dada por

$$
\begin{aligned}
\frac{\partial^{2} \ell_{p}(\boldsymbol{\theta}, \lambda)}{\partial \sigma^{4}}=\frac{\partial \mathbf{U}_{p}^{\sigma^{2}}(\boldsymbol{\theta})}{\partial \sigma^{2}} & =\frac{\partial}{\partial \sigma^{2}}\left[\frac{1}{2 \sigma^{2}}\left(\frac{(\mathbf{y}-\mathbf{X} \boldsymbol{\beta}-\mathbf{N f})^{T} \mathbf{W}(\mathbf{y}-\mathbf{X} \boldsymbol{\beta}-\mathbf{N f})}{\sigma^{2}}-\frac{n}{2}\right)\right] \\
& =-\frac{1}{\sigma^{4}}\left[\frac{(\mathbf{y}-\mathbf{X} \boldsymbol{\beta}-\mathbf{N f})^{T} \mathbf{W}(\mathbf{y}-\mathbf{X} \boldsymbol{\beta}-\mathbf{N f})}{\sigma^{2}}-\frac{n}{2}\right]
\end{aligned}
$$

que implica na informação de Fisher

$$
\mathcal{I}_{p}^{\sigma^{2} \sigma^{2}}(\boldsymbol{\theta})=-\mathrm{E}\left(\frac{\partial^{2} \ell_{p}(\boldsymbol{\theta}, \lambda)}{\partial \sigma^{4}}\right)=\frac{1}{\sigma^{4}}\left[\frac{\mathrm{E}\left[(\mathbf{y}-\mathbf{X} \boldsymbol{\beta}-\mathbf{N} \mathbf{f})^{T} \mathbf{W}(\mathbf{y}-\mathbf{X} \boldsymbol{\beta}-\mathbf{N} \mathbf{f})\right]}{\sigma^{2}}-\frac{n}{2}\right]=\frac{n}{2 \sigma^{4}} .
$$

Já a derivada de (C.4) em relação ao vetor $\boldsymbol{\beta}$ fica dada por

$$
\begin{aligned}
\frac{\partial \mathbf{U}_{p}^{\sigma^{2}}(\boldsymbol{\theta})}{\partial \boldsymbol{\beta}^{T}}=\frac{\partial^{2} \ell_{p}(\boldsymbol{\theta}, \lambda)}{\partial \sigma^{2} \partial \boldsymbol{\beta}^{T}} & =\frac{\partial}{\partial \boldsymbol{\beta}^{T}} \frac{1}{2 \sigma^{2}}\left[\frac{(\mathbf{y}-\mathbf{X} \boldsymbol{\beta}-\mathbf{N f})^{T} \mathbf{W}(\mathbf{y}-\mathbf{X} \boldsymbol{\beta}-\mathbf{N f})}{\sigma^{2}}-\frac{n}{2}\right] \\
& =-\sigma^{-2} \mathbf{N}^{T} \mathbf{W}(\mathbf{y}-\mathbf{X} \boldsymbol{\beta}-\mathbf{N f})
\end{aligned}
$$

que implica na informação de Fisher

$$
\mathcal{I}_{p}^{\sigma^{2} \boldsymbol{\beta}}(\boldsymbol{\theta})=-\mathrm{E}\left(\frac{\partial^{2} \ell_{p}(\boldsymbol{\theta}, \lambda)}{\partial \sigma^{2} \partial \boldsymbol{\beta}^{T}}\right)=\sigma^{-2} \mathbf{N}^{T} \mathbf{W E}(\mathbf{y}-\mathbf{X} \boldsymbol{\beta}-\mathbf{N f})=\mathbf{0}
$$

E, por fim, a derivada de (C.4) em relação a $\mathbf{f}$ fica dada por

$$
\begin{aligned}
\frac{\partial \mathbf{U}_{p}^{\sigma^{2}}(\boldsymbol{\theta})}{\partial \mathbf{f}}=\frac{\partial^{2} \ell_{p}(\boldsymbol{\theta}, \lambda)}{\partial \sigma^{2} \partial \mathbf{f}} & =\frac{1}{2 \sigma^{2}}\left[\frac{(\mathbf{y}-\mathbf{X} \boldsymbol{\beta}-\mathbf{N f})^{T} \mathbf{W}(\mathbf{y}-\mathbf{X} \boldsymbol{\beta}-\mathbf{N f})}{\sigma^{2}}-\frac{n}{2}\right] \\
& =-\sigma^{-2} \mathbf{X}^{T} \mathbf{W}(\mathbf{y}-\mathbf{X} \boldsymbol{\beta}-\mathbf{N f})
\end{aligned}
$$

que implica na informação de Fisher

$$
\mathcal{I}_{p}^{\sigma^{2} \mathbf{f}}(\boldsymbol{\theta})=-\mathrm{E}\left(\frac{\partial^{2} \ell_{p}(\boldsymbol{\theta}, \lambda)}{\partial \sigma^{2} \partial \mathbf{f}}\right)=\sigma^{-2} \mathbf{X}^{T} \mathbf{W E}(\mathbf{y}-\mathbf{X} \boldsymbol{\beta}-\mathbf{N f})=\mathbf{0}
$$




\section{Apêndice D}

\section{Códigos em $\mathbf{R}$}

\section{D.1 Obtenção da matriz hat no MANS}

Para rodar este programa deixe no objeto fit.model a saída do ajuste do MANS via gamlss, que será necessária para utilizarmos o mesmo parâmetro de suavização $\lambda$ do modelo ajustado nos cálculos para a obtenção da matriz hat. A saída final será a matriz H, que é a matriz $\mathbf{H}(\lambda)$ apresentada no Capítulo 2, e que será útil na construção dos gráficos de diagnóstico.

Verifica a quantidade de valores distintos da variável explicativa

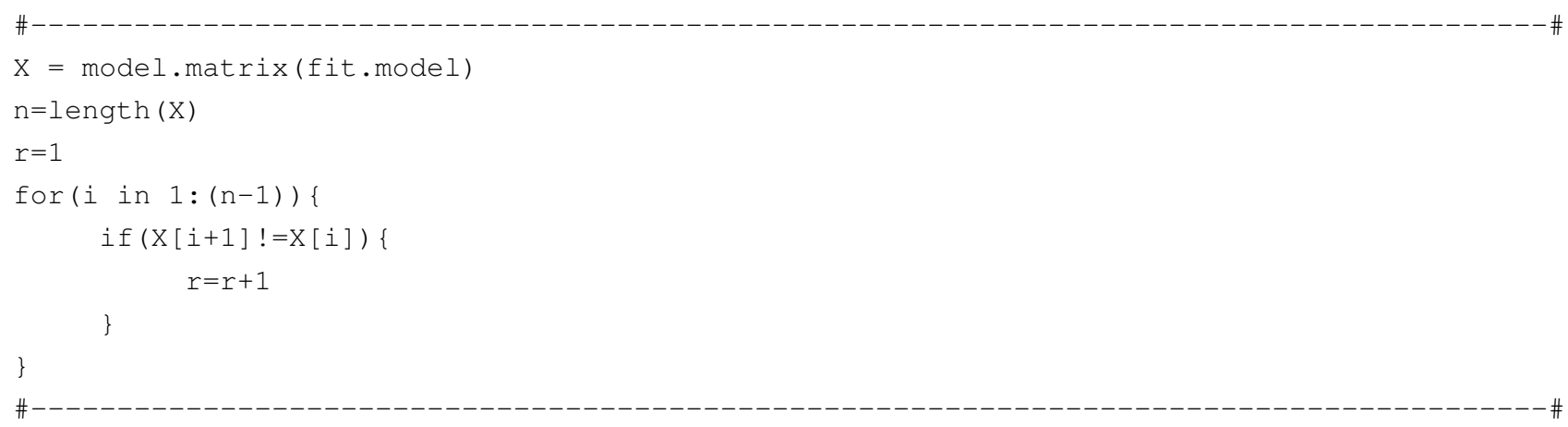

Cria o vetor de valores distintos da variável explicativa

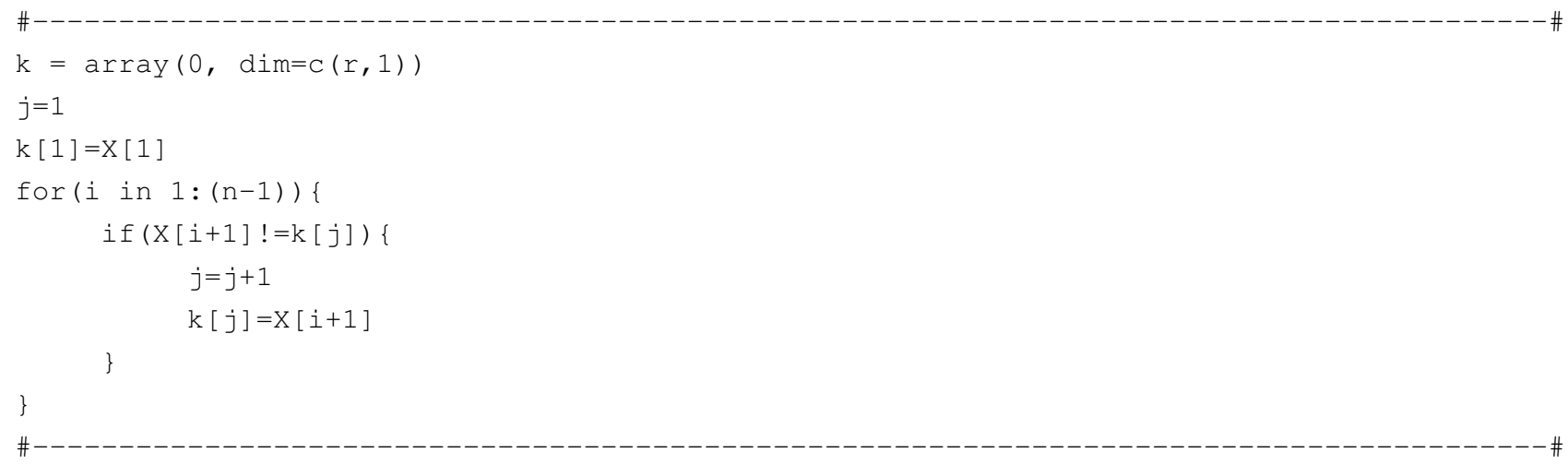

Criação da matriz de incidência $\mathbf{N}$ 


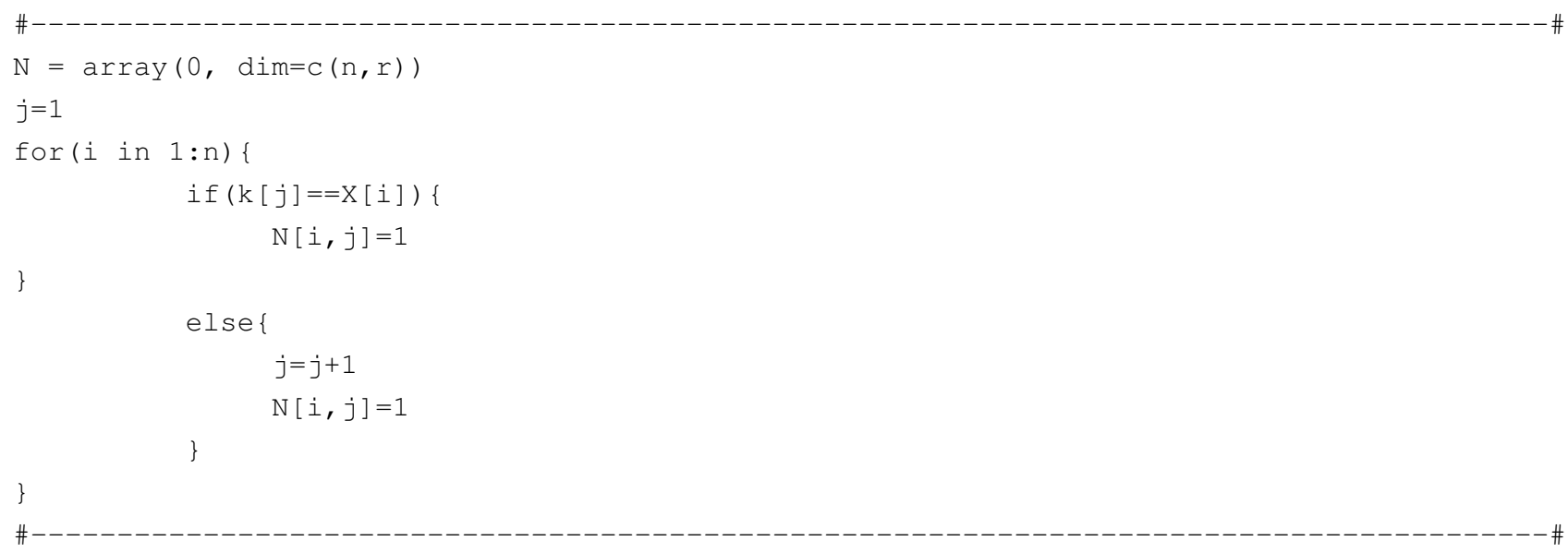

Cálculo das diferenças das observações do vetor de valores distintos da variável explicativa (útil para obtenção das matrizes $\mathbf{Q}$ e $\mathbf{R}$ )

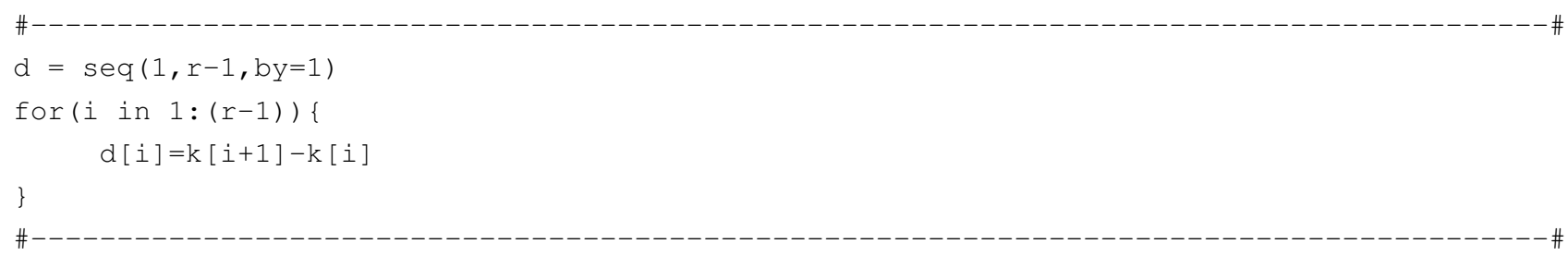

Obtenção das matrizes $\mathbf{Q}$ e $\mathbf{R}$

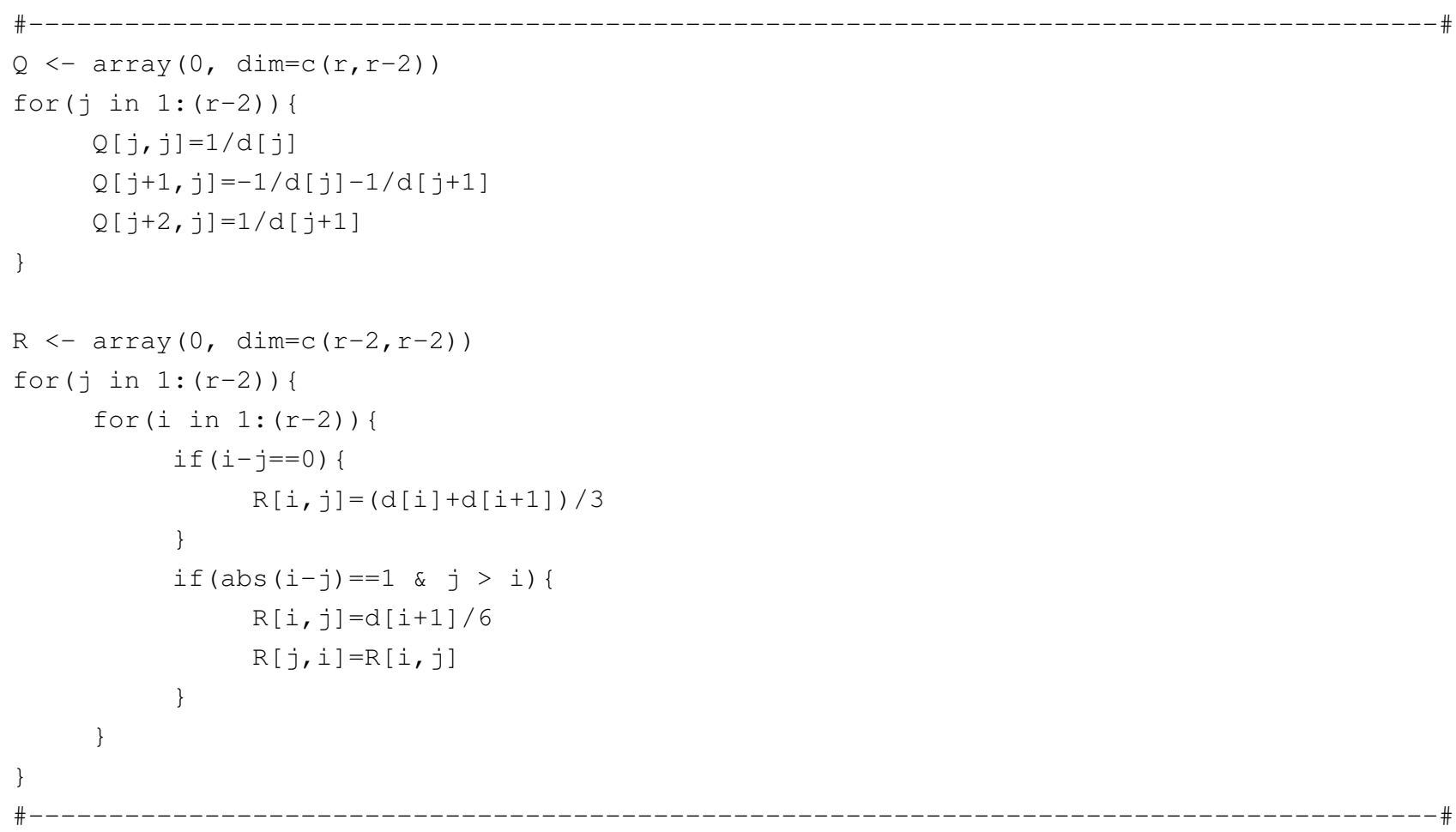

Obtenção da matriz K

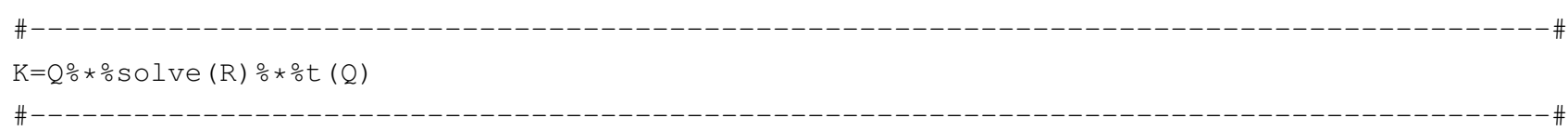


Obtenção da matriz $\mathbf{H}$

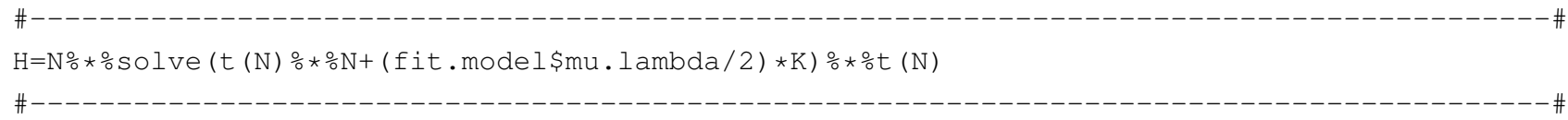

\section{D.2 Gráfico Envelope para o MANS ajustado via gamlss}

Para rodar este programa deixe no objeto fit.model a saída do ajuste do MANS ou MLP. Deixe também os dados disponíveis através do comando attach(...) e defina o número de graus de liberdade utilizados no ajuste em $g l=g l$. A saída será o gráfico de envelope para o resíduo padronizado.

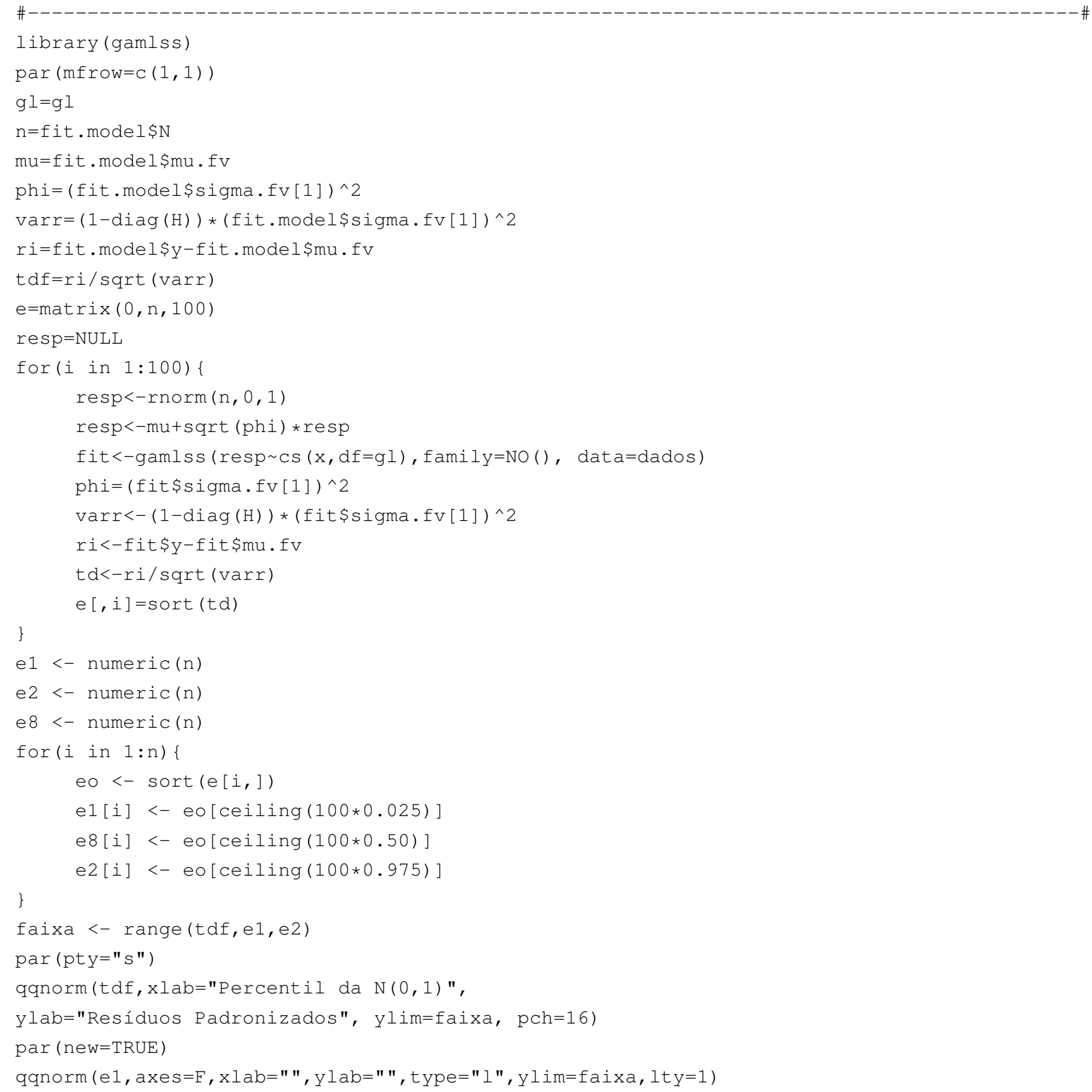


$\operatorname{par}($ new $=$ TRUE $)$

qqnorm $(e 2$, axes=F, xlab=" ",ylab=" ", type="l",ylim=faixa, lty=1)

$\operatorname{par}($ new $=$ TRUE $)$

qqnorm (e8, axes=F, xlab=" , ylab=" , type="l",ylim=faixa, lty=2)

identify (tdf, $n=2)$

\section{D.3 Gráficos de diagnóstico para o MANS ajustado via gamlss}

Para rodar este programa deixe no objeto fit.model a saída do ajuste do MANS ou MLP. Deixe os dados disponíveis através do comando attach(...). A saída terá quatro gráficos: de pontos de alavanca, de pontos, influentes e dois gráficos de resíduos. Para identificar os pontos que mais se destacam usar o comando identify(...).

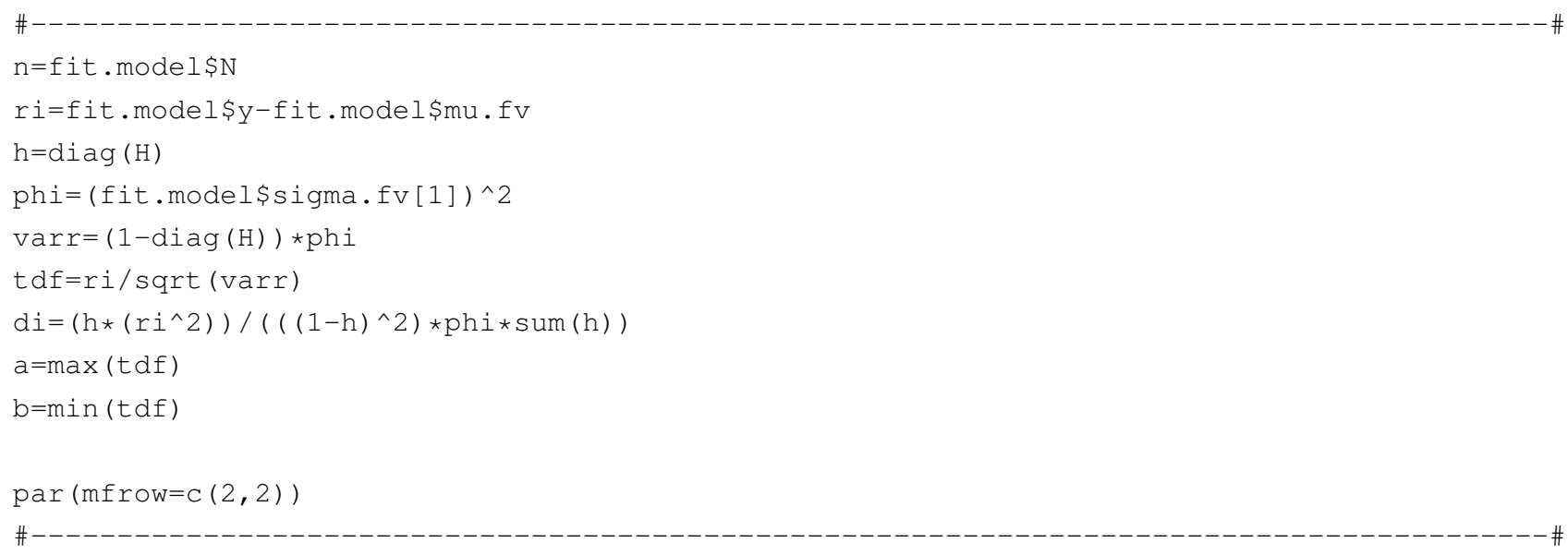

Gráfico de pontos de alavanca

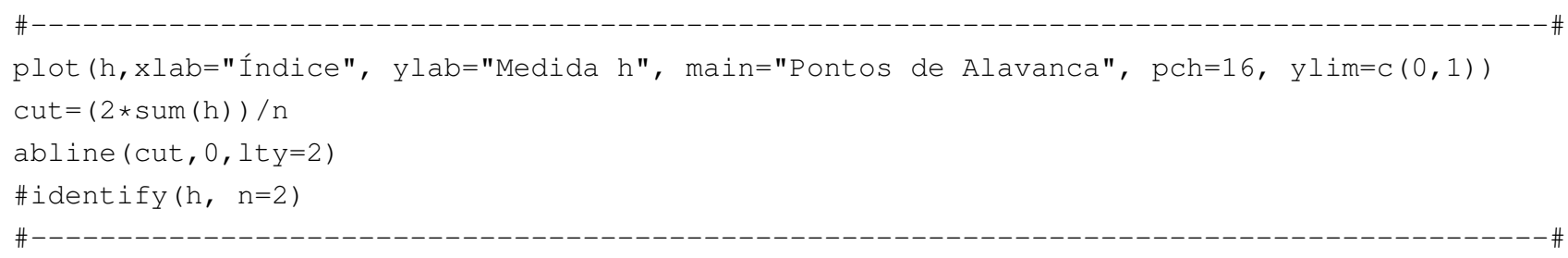

Gráfico de pontos influentes

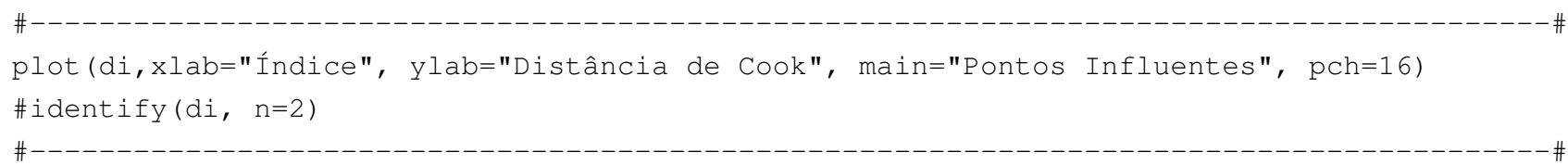

Gráfico de resíduos padronizados

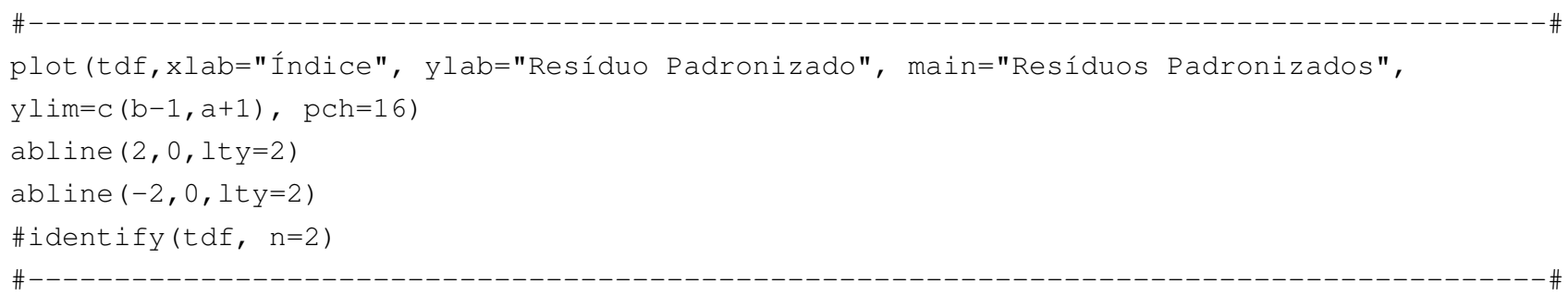


Gráfico de homocedasticidade

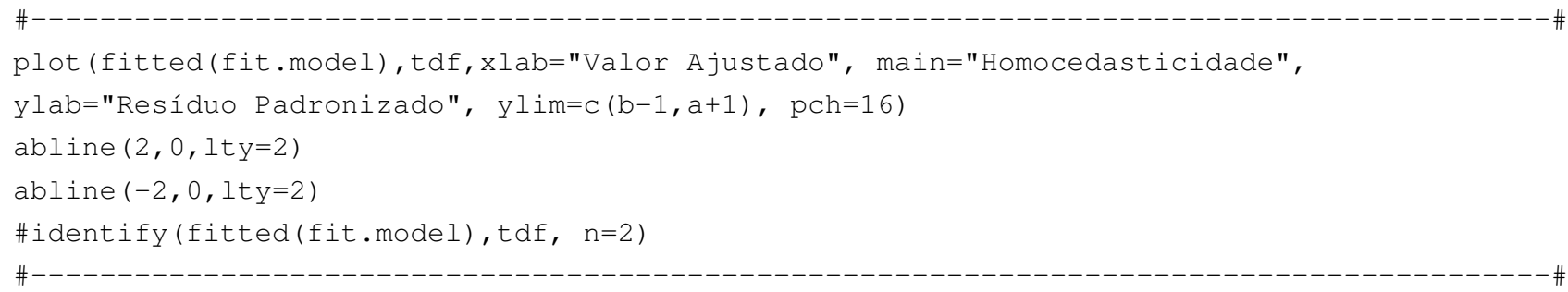

\section{D.4 Gráfico Envelope para o MLP ajustado via gam}

Para rodar este programa deixe no objeto fit.model a saída do ajuste do MLP via gam. Deixe também os dados disponíveis através do comando attach(...). A saída será o gráfico de envelope para o resíduo padronizado.

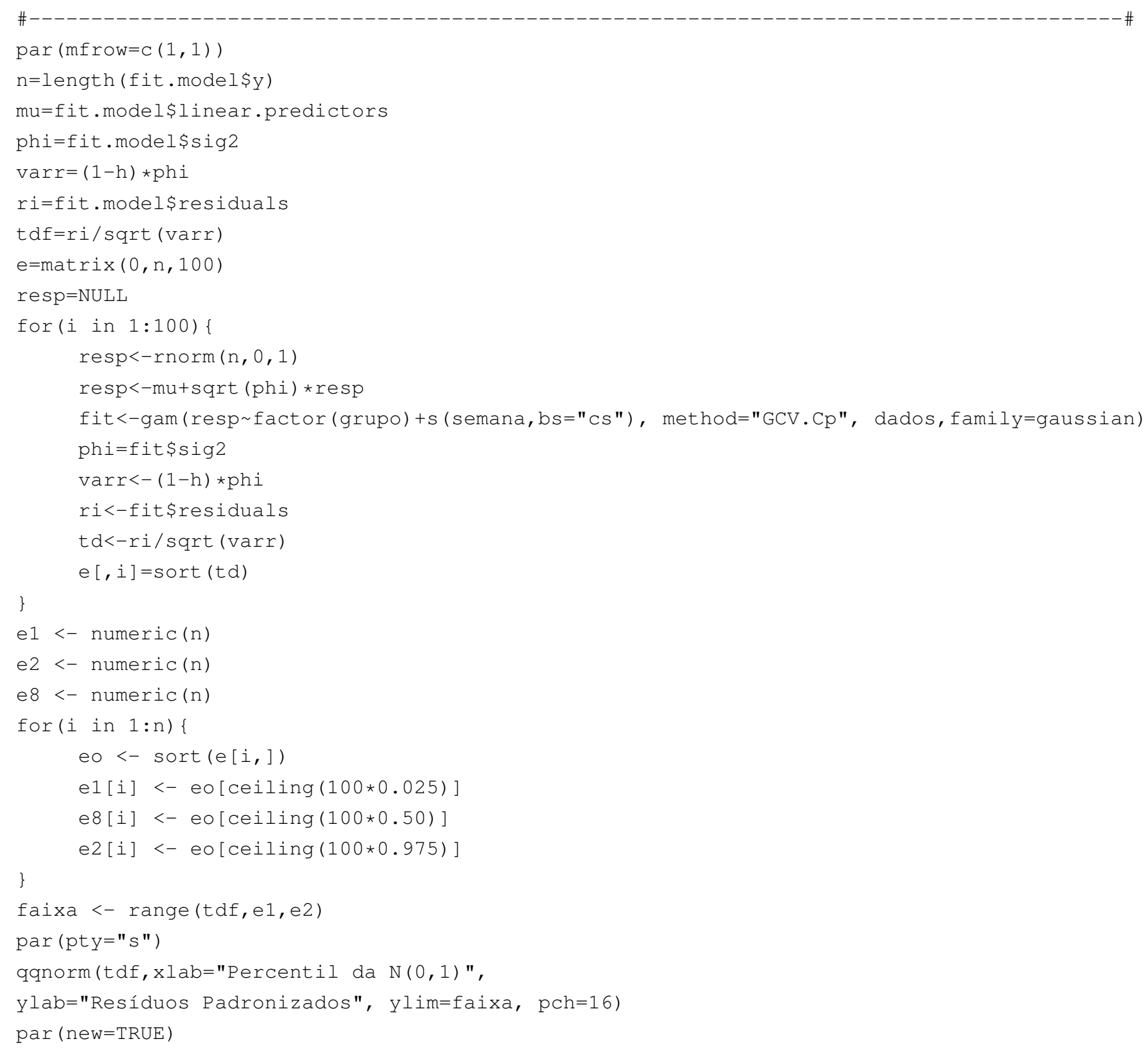


qqnorm (e1, axes=F, xlab=" ",ylab=" ", type="l ",ylim=faixa, lty=1)

$\operatorname{par}($ new $=$ TRUE)

qqnorm (e2, axes=F, xlab=" ",ylab=" ", type="l",ylim=faixa, lty=1)

par $($ new $=$ TRUE $)$

qqnorm(e8, axes=F, xlab="", ylab="", type="l",ylim=faixa, lty=2)

\section{D.5 Gráficos de diagnóstico para o MLP ajustado via gam}

Para rodar este programa deixe no objeto fit.model a saída do ajuste do MLP via gam. Deixe os dados disponíveis através do comando attach(...). A saída terá quatro gráficos: de pontos de alavanca, de pontos, influentes e dois gráficos de resíduos. Para identificar os pontos que mais se destacam usar o comando identify (...).

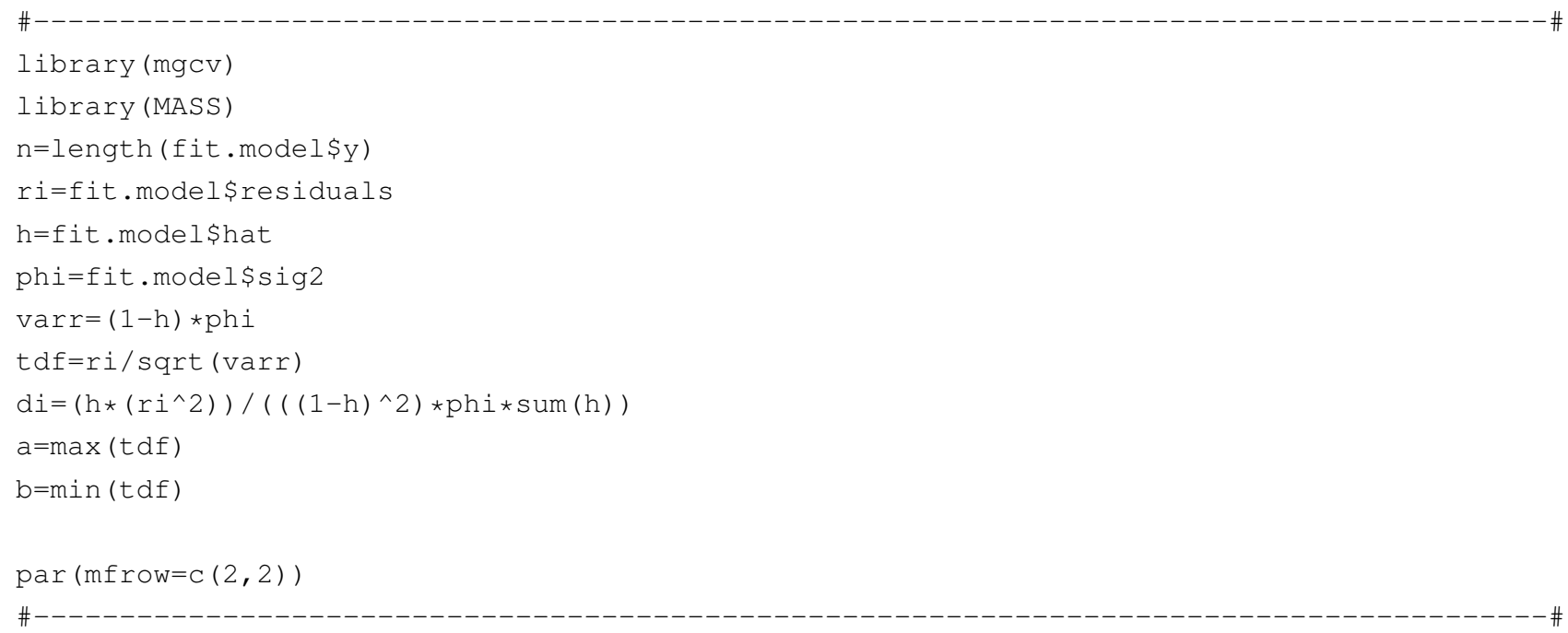

Gráfico de pontos de alavanca

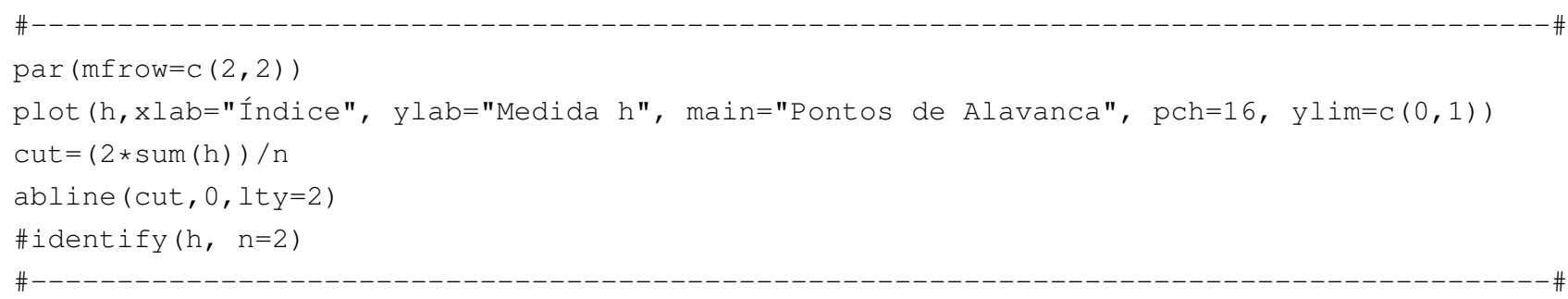

Gráfico de pontos influentes

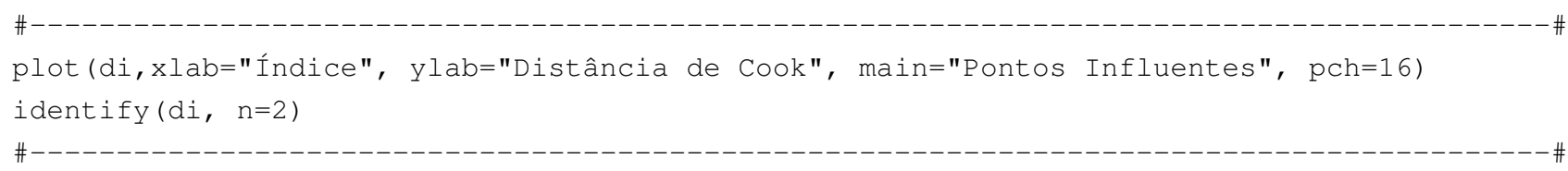

Gráfico de resíduos padronizados

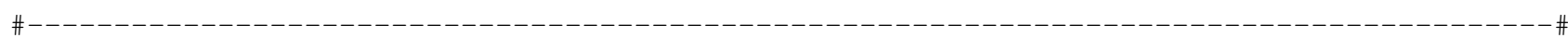

plot(tdf, xlab="Índice", ylab="Resíduo Padronizado", main="Resíduos Padronizados", ylim=c $(b-1, a+1), p c h=16)$ 
abline $(2,0,1$ ty $=2)$

abline $(-2,0,1 t y=2)$

\#identify (tdf, $n=2$ )

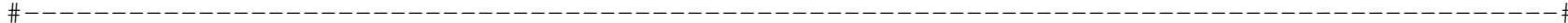

Gráfico de resíduos padronizados

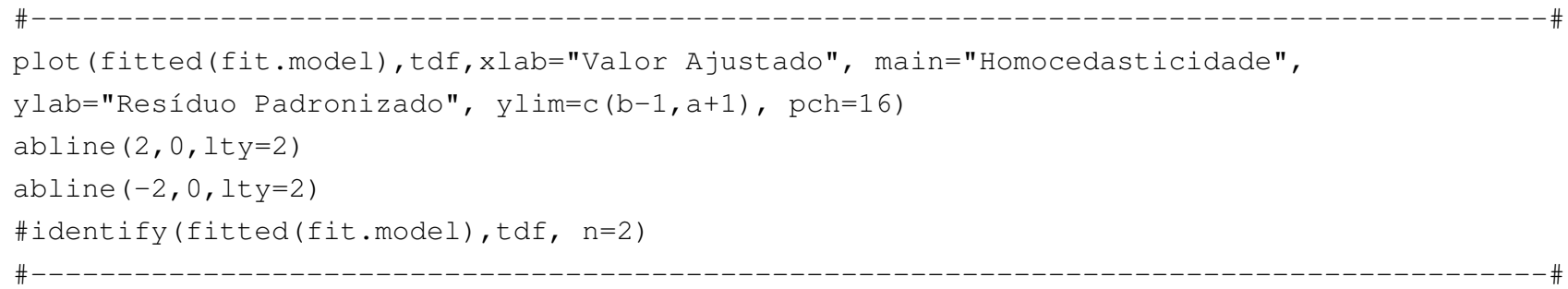


APÊNDICE D 


\section{Referências Bibliográficas}

Akaike, H. (1974), "A new look at the statistical models identification," IEEE Transactions on Automatic Control, 19, 716-722. Citado na pág. 4, 26, 40

Breiman, L. e Friedman, J. (1985), "Estimating optimal transformations for multiple regression and correlation," Journal of the American Statistical Association, 80, 580-619. Citado na pág. 34

Buja, A., Hastie, T., e Tibshirani, R. (1989), "Linear smoothers and additive models," The Annals of Statistics, 17, 453-555. Citado na pág. 9, 16, 25, 34

Cook, R. (1977), "Detection of influential observation in linear regression," Technometrics, 19, 15-18. Citado na pág. 24, 39

Craven, P. e Wahba, G. (1978), "Smoothing noisy data with spline functions," Numerische Mathematik, 31, 377-403. Citado na pág. 9

Cribari-Neto, F. e Zarkos, S. (1999), "R: Yet another econometric programming environment," Journal of Applied Econometrics, 14, 319-329. Citado na pág. 7

De Boor, C. (1978), A Practical Guide to Splines, Springer Verlag. Citado na pág. 11

Eubank, R. (1984), "The hat matrix for smoothing splines," Statistics and Probability Letters, 2, 9-14. Citado na pág. 20, 22, 23, 24, 37

- (1988), Smoothing Splines and Nonparametric Regression, Marcel Dekker, New York and Basel. Citado na pág. 9,24

Good, I. e Gaskins, R. (1971), "Nonparametric roughness penalties for probability densities," Biometrika, 58, 255-277. Citado na pág. 13

Green, P. (1987), "Penalized likelihood for general semi-parametric regression models," International Statistical Review, 55, 245-259. Citado na pág. 30, 36

Green, P., Jennison, C., e Seheult, A. (1985), "Analysis of field experiments by least squares smoothing," Journal of the Royal Statistical Society, 55, 299-315. Citado na pág. 34

Green, P. e Silverman, B. (1994), Nonparametric Regression and Generalized Linear Models: a Roughness Penalty Approach, Chapman and Hall. Citado na pág. 9, 12, 13, 25, 26, 30, 33, 34

Handscomb, D. (1966), "Spline functions," Methods of Numerical Approximation, 48. Citado na pág. $11,12,13$

Hastie, T. e Tibshirani, R. (1990), Generalized Additive Models, Chapman and Hall, London. Citado na pág. $9,25,29$ 
Heckman, N. (1986), "Spline smoothing in a partly linear model," Journal of the Royal Statistical Society. Series B (Methodological), 244-248. Citado na pág. 12, 30

Hoaglin, D. e Welsch, R. (1978), "The hat matrix in regression and ANOVA," American Statistician, $32,17-22$. Citado na pág. 23

Ibacache-Pulgar, G., Paula, G., e Cysneiros, F. (2013), "Semiparametric additive models under symmetric distributions," São Paulo: IME/USP. Citado na pág. 30

Ihaka, R. e Gentleman, R. (1996), "R: A language for data analysis and graphics," Journal of Computational and Graphical Statistics, 5, 299-314. Citado na pág. 7

Kim, C. (1996), "Cook's distance in spline smoothing," Statistics and Probability Letters, 31, 139144. Citado na pág. 9,24

Kim, C., Park, B., e Kim, W. (2002), "Influence diagnostics in semiparametric regression models," Statistics and Probability Letters, 60, 49-58. Citado na pág. 30, 37, 38, 39

Lima, L. (2001), "Modelos Aditivos Generalizados: Aplicação a um Estudo Epidemiológico Ambiental," Dissertação de Mestrado, IME/USP. Citado na pág. 25

Montgomery, D., Peck, E., e Vining, G. (2001), Introduction to Linear Regression Analysis, Wiley New York. Citado na pág. 7, 43

Neter, J., Wasserman, W., Kutner, M., e Li, W. (1996), Applied Linear Statistical Models, Irwin. Citado na pág. 20

Paula, G. (2010), "Modelos de Regressão Com Apoio Computacional," São Paulo: IME/USP. Citado na pág. 24

Paula, G., Moura, A., e Yamaguchi, A. (2004), "Relatório de análise estatística sobre o projeto: Estabilidade sensorial de "snacks"aromarizados com óleo de canola e gordura vegetal hidrogenada." Relatório do CEA, São Paulo: IME/USP. Citado na pág. 2, 7

Reinsch, C. (1967), "Smoothing by spline functions," Numerical Mathematical, 10, 177-183. Citado na pág. 11

Rigby, R. e Stasinopoulos, D. (2005), "Generalized additive models for location, scale and shape," Journal of the Royal Statistical Society, 54, 507-554. Citado na pág. 9, 25, 26

Schwarz, G. (1978), "Estimating the dimension of a model," The annals of statistics, 6, 461-464. Citado na pág. 4

Searle, S. (1982), Matrix Algebra Useful for Statistics, Wiley-Interscience. Citado na pág. 61, 65

Silverman, B. (1984), "A fast and efficient cross-validation method for smoothing parameter choice in spline regression," Journal of the American Statistical Association, 79, 584-589. Citado na pág. 11

Wahba, G. (1978), "Improper priors, spline smoothing and the problem of guarding against model errors in regression," Journal of the Royal Statistical Society, 40, 364-372. Citado na pág. 21

- (1983), "Bayesian confidence intervals for the cross-validated smoothing spline," Journal of the Royal Statistical Society. Series B (Methodological), 45, 133-150. Citado na pág. 17, 18, 19 
Wood, S. (2006), Generalized Additive Models: An Introduction With R, CRC Press. Citado na pág. 9, 18,19

Zhang, D., Lin, X., Raz, J., e Sowers, M. (1998), "Semiparametric stochastic mixed models for longitudinal data," Journal of the American Statistical Association, Theory and Methods, 93, 710-719. Citado na pág. 17 